\title{
Electrons in the DØ Central Calorimeter: A Study of the Systematic Biases in the Measurement of the W Mass
}

\author{
A Dissertation Presented \\ by \\ Terry Charles Heuring \\ to \\ The Graduate School \\ in Partial Fulfillment of the Requirements \\ for the Degree of \\ Doctor of Philosophy \\ in
}

Physics

State University of New York

at

Stony Brook

August 1993 


\section{State University of New York at Stony Brook \\ The Graduate School \\ Terry Charles Heuring}

We, the dissertation committee for the above candidate for the Doctor of Philosophy degree, hereby recommend acceptance of the dissertation.

\begin{tabular}{c} 
Advisor \\
Robert McCarthy \\
Professor \\
Department of Physics \\
\hline Michael Rijssenbeek \\
Associate Professor \\
Department of Physics \\
\\
John Smith \\
Professor \\
Department of Physics \\
\\
\hline Donald Weidner \\
Professor \\
Department of Geophysics \\
of New York at Stony Brook
\end{tabular}

This dissertation is accepted by the Graduate School.

Graduate School 


\title{
Abstract of the Dissertation Electrons in the DØ Central Calorimeter: A Study of the Systematic Biases in the Measurement of the W Mass
}

\author{
by \\ Terry Charles Heuring \\ Doctor of Philosophy \\ in \\ Physics \\ State University of New York at Stony Brook
}

1993

The DØ detector at Fermilab is a general purpose collider detector designed for the study of proton-antiproton collisions at a center of mass energy of $1.8 \mathrm{TeV}$. The detector consists of an inner tracking volume, a hermetic uranium/liquid argon calorimeter, and an outer muon detection system. Since the detector lacks a central magnetic field, it relies on energy measurements from the calorimeter as opposed to momentum measurements using the tracking chambers.

To provide the necessary understanding of the calorimeter, a testbeam was conducted at Fermilab during the second half of 1991 featuring detector modules from the central calorimeter. Detailed simulations of the detector 
apparatus were also written. This thesis will present the results of this testbeam and simulation effort and relate them to the measurement of the $W^{ \pm}$ intermediate vector boson mass in the full $\mathrm{D} \emptyset$ detector.

In the testbeam, an energy resolution that scaled as $14 \%$ divided by the square root of the beam energy was found. The uniformity of response of the detector as a function of angle of incidence was investigated. We found that the response increased by $4 \%$ over the range investigated. The results were compared to a simulation written using the CERN package GEANT. Although GEANT was able to reproduce the energy resolution, it was not able to reproduce the uniformity of response function. A second simulation utilizing the EGS4 package from SLAC was successful in reproducing the behavior of the detector as a function of angle. The biases induced by the discrepancies between the detector and GEANT response functions in the $\mathrm{W}^{ \pm}$mass measurement are studied. We find that using GEANT as a detector simulation will cause a bias of between 460 and $680 \mathrm{MeV}$ in the $\mathrm{W}^{ \pm}$mass determination. 
This thesis is dedicated to my family, especially my parents. 


\section{Contents}

List of Figures .................... xiii

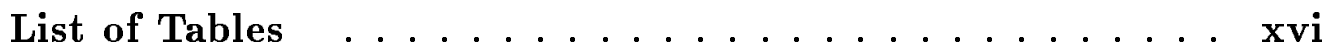

Acknowledgements ................. xvii

1 Introduction .................... 1

2 Physics at $D \emptyset \ldots \ldots \ldots \ldots$

2.1 The Standard Model ................. 4

$2.2 \quad$ Properties of $\mathrm{W}^{ \pm} \quad \ldots \ldots \ldots \ldots \ldots$

2.3 Measuring $M_{W}$................... 10

2.4 Electromagnetic Showers . . . . . . . . . . . . 15

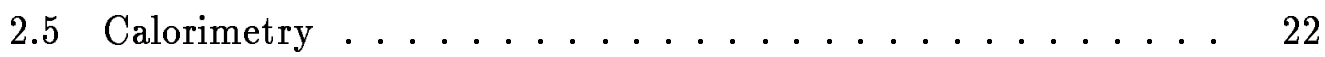

3 The DØ Detector ................... 31

3.1 Detector Overview .................. 31

3.2 Uranium/Liquid Argon Calorimeters . . . . . . . . . . 35

3.2.1 General Design . . . . . . . . . . . . . 35

3.2.2 The Central Calorimeter . . . . . . . . . . . 38 
3.2.3 Calorimeter Electronics . . . . . . . . . . . . . . 40

3.2.4 Trigger and Data Acquisition . . . . . . . . . 42

4 The 1991 Testbeam $\ldots \ldots \ldots \ldots \ldots \ldots \ldots$

4.1 Testbeam goals $\ldots \ldots \ldots \ldots \ldots \ldots \ldots \ldots$

4.2 Testbeam Configuration $\ldots \ldots \ldots \ldots \ldots$

4.3 Testbeam Simulation . . . . . . . . . . . . 50

$5 \quad$ Energy Scans $\ldots \ldots \ldots \ldots \ldots \ldots \ldots \ldots$

5.1 Data $\operatorname{Set} \ldots \ldots \ldots \ldots \ldots \ldots \ldots \ldots$

5.2 Monte Carlo Data Set . . . . . . . . . . . . . 57

5.3 Energy Resolution . . . . . . . . . . . . 61

$5.4 \quad$ Results . . . . . . . . . . . . . . . . 62

$6 \eta \operatorname{Scan} \ldots \ldots \ldots \ldots \ldots \ldots \ldots \ldots \ldots \ldots \ldots$

6.1 Testbeam Data Set . . . . . . . . . . . . . . 74

6.2 Monte Carlo Data Set . . . . . . . . . . . 76

6.3 Comparison of Monte Carlo and Data . . . . . . . . 78

7 EGS4 - GEANT 3.14 Comparison $\ldots \ldots \ldots \ldots$

7.1 Geometry . . . . . . . . . . . . . . . 80

7.2 Results at $10 \mathrm{GeV} \ldots \ldots \ldots \ldots$. . . . . . . 81

7.3 Results at $100 \mathrm{GeV} \ldots \ldots \ldots \ldots$. . . . . . . 84

7.4 Results Using $\delta$ Rays . . . . . . . . . . . . . . . . . 87 
8 W Mass Measurement .................. 90

8.1 Event Simulation . . . . . . . . . . . . . . . 91

8.2 Collider Data Set . . . . . . . . . . . . . . 102

8.3 Results ..................... 105

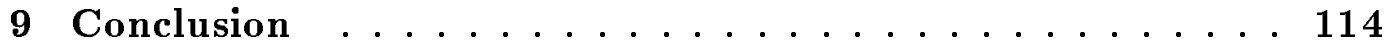

A Trigger and Analysis Cuts . . . . . . . . . . 121

A.1 Level 1 Trigger Requirements . . . . . . . . . . . . . . 121

A.2 Level 2 Trigger Requirements . . . . . . . . . . . . . . 122

A.3 Analysis Requirements . . . . . . . . . . . . . . . 124 


\section{List of Figures}

2.1 Example of quantum loop corrections to the $\mathrm{W}^{ \pm}$and $\mathrm{Z}^{0}$ boson mass. .................. 8

2.2 Relationship between $M_{Z}$ and $M_{W}$ in the minimal Standard Model for various top masses. . . . . . . . . . . .

2.3 Production of the $\mathrm{W}^{ \pm}$boson from $\overline{\mathrm{p}} \mathrm{p}$ collisions and subsequent decay into an electron and neutrino. . . . . . . . . .

2.4 Cross sections for various processes in carbon, iron, and uranium as a function of particle energy for both photons $\left(E_{\gamma}\right)$ and

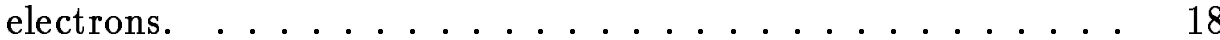

2.5 The electromagnetic shower development as a function of depth for various materials using the EGS4 Monte Carlo showing the approximate scaling of the longitudinal profile when expressed

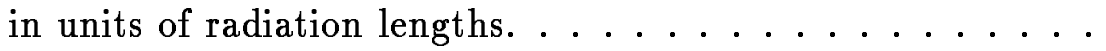

2.6 The mass attenuation coefficients for photons in different materials as a function of energy $\left(E_{\gamma}\right) \ldots \ldots \ldots 23$ 
2.7 The effects of both longitudinal and transverse leakage on the energy resolution as measured for electrons in the CHARM neutrino calorimeter. . . . . . . . . . . . . . 26

2.8 The e/mip ratio for liquid argon and scintillator calorimeters as a function of the $\mathrm{Z}$ of the absorbing medium. . . . . . . . 28

2.9 The e/mip ratio for liquid argon and scintillator calorimeters for various uranium absorber plate thicknesses. . . . . . . . 30

3.1 The DØ Detector. . . . . . . . . . . . . . 32

3.2 Cutaway View of the DØ Calorimeters . . . . . . . . 36

3.3 The standard unit cell used in the DØ central calorimeter. . . 37

3.4 The pad design used in the central calorimeter signal boards. . 40

3.5 A quarter view of the $\mathrm{D} \emptyset$ calorimeter showing the coverage in $\eta .41$

4.1 Beamline used in the 1991 testbeam. . . . . . . . . . . . 47

4.2 Configuration of the 1991 testbeam. . . . . . . . . . . 48

4.3 The cryostat and transporter system used in the 1991 testbeam. 49

5.1 Response vs. the extrapolation of the reconstructed vertical track into the third electromagnetic layer for run $13842(20 \mathrm{GeV}) .56$

5.2 Response in the first electromagnetic section vs. the extrapolation of the reconstructed vertical track into the third section for run $13842(20 \mathrm{GeV}) . \ldots \ldots \ldots$ 
5.3 Response vs. the extrapolation of the reconstructed horizontal track into the third electromagnetic section for run $13842(20$ $\mathrm{GeV}) . \ldots \ldots \ldots \ldots \ldots \ldots$

5.4 Comparison of the simulated and real data resolutions for $\eta=$

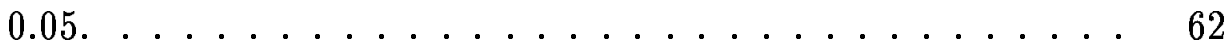

5.5 Comparison of the simulated and real data resolutions for $\eta=$

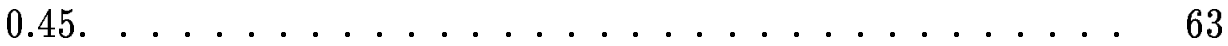

5.6 Comparison of the simulated and real data resolutions for $\eta=$

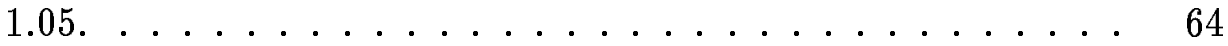

5.7 Comparison of the simulated and noise subtracted data resolutions for $\eta=0.05 \ldots \ldots \ldots \ldots \ldots$. . . . . . . 65

5.8 Comparison of the simulated and noise subtracted data resolutions for $\eta=0.45 \ldots \ldots \ldots \ldots \ldots 6$

5.9 Comparison of the simulated and noise subtracted data resolutions for $\eta=1.05 \ldots \ldots \ldots \ldots$. . . . . . . . 67

6.1 Beam projections into the testbeam cryostat for the $\eta$ scan. .

6.2 Response vs. $\eta$ for $100 \mathrm{GeV}$ electrons for both the testbeam data and the simulation. . . . . . . . . .

6.3 Energy deposition by layer for the four electromagnetic layers as a function of pseudorapidity for both testbeam data and the

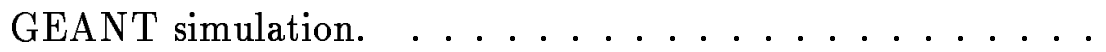

7.1 Geometrical configuration used in the comparison of EGS4 and GEANT 3.14. . . . . . . . . . . . . 82 
7.2 Results of the $\eta$ scan for both EGS4 and GEANT 3.14 using $10 \mathrm{GeV}$ electrons. No upstream correction factor (22\%) was applied to layer $1 . \ldots \ldots \ldots$

7.3 Comparison of EGS4 and GEANT 3.14 using $10 \mathrm{GeV}$ electrons. Results are plotted vs. $\eta$ for the four electromagnetic layers. .

7.4 Results of the $100 \mathrm{GeV} \eta$ scan for testbeam data and both simulations. ................... 86

7.5 Comparison of EGS4 and GEANT 3.14. The delta rays in GEANT are explicitly tracked down to $10 \mathrm{KeV}$. . . . . . . 88

8.1 Distribution of the difference between the reconstructed electron $\eta$ and the true electron track. . . . . . . . . . . 94

8.2 Distribution of the difference between the reconstructed electron $\phi$ angle and the true electron track. . . . . . . . . .

8.3 The mean $E_{T}$ minus the $E_{T}$ of the decay electron plotted vs.

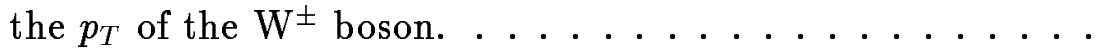

8.4 The probability of an event having two jets as a function of the sum of the $E_{T} . \ldots \ldots \ldots \ldots \ldots \ldots$

8.5 $Z_{T}$ resolution as a function of total scalar $E_{t}$ as derived from minimum bias events. . . . . . . . . . . . . . 100

8.6 $Z_{T}$ resolution as a function of total scalar $E_{t}$ as derived from QCD events....................... 101

8.7 Fit for $m_{T}$ with the testbeam response function. . . . . . . . 106

8.8 Fit for $p_{T}(e)$ with the testbeam response function. . . . . . . 107 
8.9 Fit for $p_{T}(\nu)$ with the testbeam response fucntion. . . . . . . 108

8.10 Fit for $m_{T}$ with the GEANT $\eta$ response function. . . . . . . 109

8.11 Fit for $p_{T}(e)$ with the GEANT $\eta$ response function. . . . . . . 110

8.12 Fit for $p_{T}(\nu)$ with the GEANT $\eta$ response function. . . . . . . 111 


\section{List of Tables}

2.1 Fermions of the Standard Model. ............. 5

2.2 Bosons of the Standard Model. . . . . . . . . . . . 6

2.3 Experimentally measured values for the intermediate vector boson masses and their associated errors. . . . . . . . . . 9

2.4 Uncertainties from various sources for the $\mathrm{W}^{ \pm}$boson mass in the minimal Standard Model. . . . . . . . . . . . . . 10

2.5 A summary of the UA2 corrections and systematic errors for each of the $W$ mass fits. . . . . . . . . . . .

2.6 Electromagnetic shower development showing the number and type of particles as well as the average energy as a function of

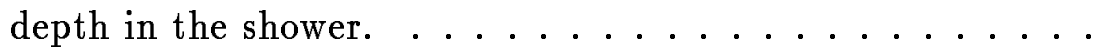

3.1 Design parameters for the central calorimeter modules. . . . . 38

3.2 Physics design parameters of the central calorimeter modules. $\quad 39$

4.11991 Testbeam Load 2 Beamline Parameters. . . . . . . . . . . 51

4.2 Location of the beamline elements relative to the front of the testbeam cryostat. ................ 51 
4.3 Elements upstream of the calorimeter modules inside the testbeam cryostat. .................. . . 52

4.4 Dimensions of the elements modeled in the CCEM module. . . 53

4.5 Dimensions of the elements modeled in the CCFH module. . . 53

5.1 Events remaining after cuts applied to the $\eta=0.05$ dataset. . 68

5.2 Events remaining after cuts applied to the $\eta=0.45$ dataset. . $\quad 69$

5.3 Events remaining after cuts applied to the $\eta=1.05$ dataset. . $\quad 69$

5.4 Events remaining after cuts for the Monte Carlo data at $\eta=$

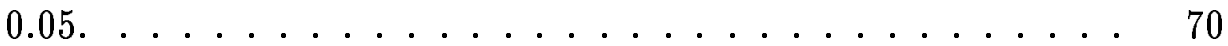

5.5 Events remaining after cuts for the Monte Carlo data at $\eta=$

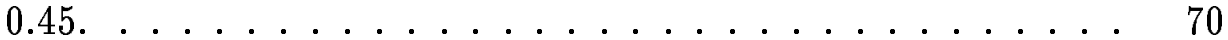

5.6 Events remaining after cuts for the Monte Carlo data at $\eta=$

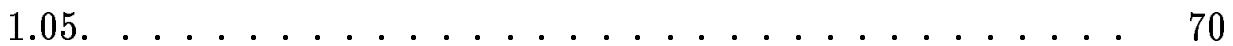

5.7 Response and resolution of the final datasets for $\eta=0.05 . \quad$. . 71

5.8 Response and resolution of the final datasets for $\eta=0.45 . \quad$. $\quad 71$

5.9 Response and resolution of the final datasets for $\eta=1.05$. . . 72

5.10 Response and resolution of the Monte Carlo data at $\eta=0.05 . \quad 72$

5.11 Response and resolution of the Monte Carlo data at $\eta=0.45 . \quad 72$

5.12 Response and resolution of the Monte Carlo data at $\eta=1.05 . \quad 73$

5.13 Results of fits to $\frac{\sigma}{\mu}=\sqrt{C^{2}+\frac{S^{2}}{E}+\frac{N^{2}}{E^{2}}}$......... 73

6.1 Tracking requirements imposed on the $\eta$ scan data set. . . . . 76 
7.1 Total live energies weighted by sampling fractions for the three Monte Carlo data sets. . . . . . . . . . . . . . . 89

8.1 The $\eta$ dependent response function $\mathrm{R}$ used to smear the Monte Carlo data sample $M C_{T B}$. . . . . . . . . . . . . . 103

8.2 The $\eta$ dependent response function $\mathrm{R}$ used to smear the Monte Carlo data sample $M C_{\eta}$ and $M C_{\eta}^{\prime}$. . . . . . . . . . 103

8.3 Triggering requirements for the $\mathrm{W}^{ \pm}$data. . . . . . . . . . 104

8.4 Results for the $M_{W}$ fits both with and without the $\eta$ resolution functions. .................... 112

8.5 Systematic shifts in $M_{W}$ introduced by the $\eta$ response function. 113 


\section{Acknowledgements}

I can't hope to acknowledge everyone who helped me complete this thesis. Therefore, I will apologize now for those that I've missed. I certainly want to thank the physicists and technicians who played a part in building $D \emptyset$. When the papers are being published we often forget the efforts of those that built the detector. The same can be said of those that worked on the testbeam. Without all of their efforts, this thesis, not to mention the experiment, would not have been possible.

I'd like to single out a few individuals whose contributions were special. First of all, I'd like to thank my advisor, Prof. Bob McCarthy, for his guidance and for reading this thesis more times than he would have liked. I'd also like to thank Marcel Demarteau for our discussions on the testbeam simulation. The completion of this thesis was helped along by Norman Graf's good natured prodding. The effort of W. G. D. Dharmaratna (Dharma) and Srini Rajagopalan in both the $\eta$ scan and the $\mathrm{W}^{ \pm}$mass studies were crucial to my analyses. However, none of this would have been possible without our spokesperson Prof. Paul Grannis.

Bob Hirosky deserves a special acknowledgement. Our many discussions 
on the flux issue and our attempts to solve the problem helped maintain my sanity. I'd like to also thank Rich Astur, my roommate for so many years. If we only cleaned as well as we built detectors.... I'd also like to acknowledge all of those who worked in both the test tent and the clean room. It was often a hard and thankless job, but I believe that our efforts have paid off. I'd like to thank all the other graduate students that suffered alongside me, especially those from SUNY Stony Brook, for their support and friendship.

Finally, I think Bob would agree that Hillary deserves a thank-you for all the free beer. 


\section{Chapter 1}

\section{Introduction}

The goal of contemporary collider experiments is to advance our knowledge of the subatomic world through both the precision testing of the currently accepted theories and the search for new phenomena beyond these theories. To accomplish this goal, detector systems such as the DØ detector[1] have been optimized to study $\overline{\mathrm{p}} \mathrm{p}$ collisions at a center of mass energy of $1.8 \mathrm{TeV}$, now available at the Fermilab Tevatron. These systems have grown greatly in complexity over those of earlier experiments. Calorimeters, devices that measure the energy of particles through absorbtion(Sec. 2.5), have become mammoth detectors weighing hundreds of tons with tens of thousands of in-

dividual electronics channels. In order to make the relevant measurements, experimenters need to understand in great detail the behavior of their apparatus. For measurements not limited by statistics, systematic errors need to be controlled.

One part of understanding the detector involves the use of testbeams, the exposure of detectors to particle beams of known characteristics under 
controlled conditions. DØ has conducted a testbeam experiment that included a wedge of the calorimeter representing approximately $\frac{1}{8}$ of the full detector and incorporating models of various dead materials. This configuration was illuminated with monochromatic beams of pions, muons, and electrons with energies from 2 to $150 \mathrm{GeV}$. Energy calibrations and response functions were determined.

A second aspect to understanding the detector includes Monte Carlo simulations, models of the detectors that reproduce their behavior. These simulations allow for extrapolations of testbeam measurements to be made. For the testbeam configuration, a detailed model was constructed. The behavior of this simulated calorimeter was compared with the real one. Together, these two efforts allow for meaningful physical measurements to be made. For example, the determination of the mass of the $\mathrm{W}$, an intermediate vector boson, relies heavily on both the detector and the simulation.

This thesis will report on the results of the testbeam experiment and a comparison to the Monte Carlo simulation concentrating on electron response and its implications on the measurement of the $\mathrm{W}$ mass.

Chapter 2 will provide an overview necessary to understand some of the motivations of the $\mathrm{D} \emptyset$ collaboration through a brief discussion of the standard model, a discussion of electromagnetic showers and their behavior in calorimeters, and a presentation of the methodology used to determine the $\mathrm{W}$ mass.

Chapter 3 will provide a description of the $D \emptyset$ detector with a detailed description of the calorimeter.

Chapter 4 will discuss the details of the testbeam including its motivation 
and design details and also provide an in depth discussion of the design of the computer simulations.

The following chapters, $5-7$, will present the results from the testbeam and the subsequent comparisons to simulations on electron energy resolution and uniformity of response. Comparisons are made between two of the most accepted simulation packages available today. Chapter 8 will explore how the results of the earlier chapters affect $D \emptyset$ 's measurement of the $\mathrm{W}$ mass. 


\section{Chapter 2}

\section{Physics at DØ}

\subsection{The Standard Model}

Particle physicists are currently occupied doing experiments that test the theory known as the Standard Model. This theory is the currently accepted model of the interactions of fundamental forces and particles. With its framework laid down more than 20 years ago, the theory has survived all tests done to date. With the advent of higher energy colliders, these tests will continue and probe ever deeper into details of the theory[2].

In the Standard Model, there are two classes of particles, fermions and bosons. The fermions are half-integral spin objects that obey Fermi-Dirac statistics. The bosons have integral spin and obey Bose-Einstein statistics. Fermions are the basic constituents of what we know as matter. Bosons are responsible for transmitting forces between fundamental particles.

Fermions are grouped into two classes, quarks and leptons. Each type has a set of quantum numbers that governs its behavior. In addition, both types of 


\begin{tabular}{||lcccc||}
\hline Particle & Symbol & Charge & Mass $(\mathrm{GeV})$ & \\
\hline Electron neutrino & $\nu_{e}$ & 0 & $<0.94 \times 10^{-8}$ & \\
Electron & $e$ & -1 & $0.51 \times 10^{-3}$ & $1^{\text {st }}$ \\
Up quark & $u$ & $2 / 3$ & $5 \times 10^{-3}$ & generation \\
Down quark & $d$ & $-1 / 3$ & $9 \times 10^{-3}$ & \\
\hline Muon neutrino & $\nu_{\mu}$ & 0 & $<0.25 \times 10^{-3}$ & \\
Muon & $\mu$ & -1 & 0.106 & $2^{\text {nd }}$ \\
Charm quark & $c$ & $2 / 3$ & 1.25 & generation \\
Strange quark & $s$ & $-1 / 3$ & 0.175 & \\
\hline Tau neutrino & $\nu_{\tau}$ & 0 & $<0.035$ & \\
Tau & $\tau$ & -1 & 1.78 & $3^{\text {rd }}$ \\
Top quark & $t$ & $2 / 3$ & $>91$ & generation \\
Bottom quark & $b$ & $-1 / 3$ & 4.3 & \\
\hline
\end{tabular}

Table 2.1: Fermions of the Standard Model[2].

fermions appear in a repeated substructure grouped into doublets each known as a generation. The model itself has nothing to say as to the number of such generations or the fermion masses, which must be measured experimentally. Table 2.1 shows the fermions grouped into their pairs of doublets.

These two classes of fermions also differ in the quantum numbers that they carry that determine the interactions in which they take part. For example, both quarks and leptons can carry electric charge and take part in electroweak interactions. However, quarks posses a quality known as color allowing them to participate in the strong interaction while leptons do not.

The fundamental bosons are shown in table 2.2. The discovery in 1982 of the intermediate vector bosons (IVB), $\mathrm{W}^{ \pm}$and $\mathrm{Z}^{0}$, was one of the triumphs of the Standard Model[3][4][5][6]. It was confirmation of the Glashow-WeinbergSalam theory $[7]$ that unified the electromagnetic and weak forces. Four different bosons participate in electroweak interactions, $\mathrm{W}^{ \pm}, \mathrm{Z}^{0}$ and $\gamma$ with 


\begin{tabular}{||lccc||}
\hline Particle & Symbol & Charge & Mass $(\mathrm{GeV})$ \\
\hline Photon & $\gamma$ & $\mathbf{0}$ & $\mathbf{0}$ \\
W boson & $\mathrm{W}^{ \pm}$ & \pm 1 & $80.14 \pm 0.31$ \\
Z boson & $\mathrm{Z}^{0}$ & $\mathbf{0}$ & $91.17 \pm 0.02$ \\
Gluon & $g$ & $\mathbf{0}$ & $\mathbf{0}$ \\
Higgs & $\boldsymbol{H}$ & $\mathbf{0}$ & $48 \lesssim m_{H} \lesssim 1000$ \\
\hline
\end{tabular}

Table 2.2: Bosons of the Standard Model[2]. All have spin 1 except for Higgs which has spin 0.

$\alpha\left(\alpha=\frac{1}{137}\right)$, the fine structure constant, and $\sin ^{2} \theta_{W}$, the sine of the weak mixing angle, being the couplings constant associated with these interactions. Exact local gauge invariance would result in all four electroweak bosons having the same mass. However, through a process known as spontaneous symmetry breaking[8], three of the bosons acquire mass with the photon remaining massless. This process predicts that there is another physically observable particle known as the Higgs boson. Current searches have shown it to be heavier than $48 \mathrm{GeV}[9]$. The Standard Model allows for it to be quite heavy, $1 \mathrm{TeV}$, before theoretical problems arise[2].

The other class of bosons, the gluons, are responsible for transmitting the strong force. The quantum number associated with this interaction has been given the name color. Hence the theory governing their interactions is known as quantum chromodynamics (QCD). The coupling constant in this theory is $\alpha_{s}$. Like the photon in quantum electrodynamics (QED), gluons are massless[2]. However, unlike photons, gluons carry a color charge and in principle can interact strongly with each other.

To date, all of the quarks with the exception of the top have been observed. The Collider Detector at Fermilab (CDF) placed a lower limit of $91 \mathrm{GeV}$ on 
this quark based on the data taken during its last run[10]. Other experiments and theoretical considerations suggest that $\mathrm{m}_{\mathrm{t}} \simeq 130 \pm 40 \mathrm{GeV}[2]$. Finding this last elusive quark is one of the paramount goals of current particle physics experiments at Fermilab. However, precision measurements of Standard Model parameters are also a primary concern.

The extremely precise measurement of the $Z^{0}$ mass made at CERN's Large Electron Positron storage ring (LEP) has opened the door to a model testing experiment in measuring the $\mathrm{W}^{ \pm}$mass. Calculations of the one loop radiative corrections to the IVB masses[2] have been made (Fig. 2.1). Through loops involving the top, precise measurements of the $\mathrm{W}^{ \pm}$mass coupled with LEP's $\mathrm{Z}$ mass constrain the top mass. $\mathrm{A} \mathrm{W}^{ \pm}$mass measurement to $\pm 50 \mathrm{MeV}$ will determine the top mass to $\pm 10 \mathrm{GeV}$ modulo uncertainties due to the Higgs boson[2]. Coupled with the expected discovery of the top during this or the next run at Fermilab, the $\mathrm{W}^{ \pm}$mass measurement will provide a powerful check at the quantum loop level of the standard model and perhaps provide information on the Higgs boson.

\subsection{Properties of $\mathrm{W}^{ \pm}$}

As discussed in section 2.1, the intermediate vector bosons play a fundamental role in the Standard Model. Precision measurements of model parameters are providing the predictive power that may show inconsistencies within the model. Determination of the $\mathrm{Z}^{0}$ mass to $20 \mathrm{MeV}$ by the four LEP experiments[11] provides the cornerstone for subsequent studies. Further mea- 
Figure 2.1: Example of quantum loop corrections to the $\mathrm{W}^{ \pm}$and $\mathrm{Z}^{0}$ boson mass. 


\begin{tabular}{||ccl||}
\hline Boson & Experiment & Mass \\
\hline$M_{Z}$ & LEP & $91.173 \pm \mathbf{0 . 0 2 0}$ \\
$M_{W}$ & UA2 & $80.79 \pm .31($ stat $) \pm .21($ sys $) \pm .81($ scale $)$ \\
$M_{W}$ & CDF & $79.91 \pm .35($ stat $) \pm .24($ sys $) \pm .19($ scale $)$ \\
\hline
\end{tabular}

Table 2.3: Experimentally measured values for the intermediate vector boson masses and their associated errors.

surements, such as the mass of the $\mathrm{W}^{ \pm}$, must be made with sufficient accuracy to proceed to test the model.

In the Standard Model with one Higgs doublet, frequently called the minimal Standard Model, the masses of the bosons can be written as[12]:

$$
\begin{aligned}
M_{W}^{2} & =\frac{A^{2}}{(1-\Delta r) \sin ^{2} \theta_{W}} \\
M_{Z}^{2} & =\frac{A^{2}}{(1-\Delta r) \sin ^{2} \theta_{W} \cos ^{2} \theta_{W}}
\end{aligned}
$$

where:

$$
\begin{gathered}
A^{2}=\frac{\pi \alpha}{\sqrt{2} G_{\mu}} \\
\theta_{W}=\text { the weak mixing angle } \\
\Delta r=\text { radiative corrections }
\end{gathered}
$$

The values for $\alpha$, the fine structure constant, and $G_{\mu}$, the muon decay constant, are known very precisely from other sources. The radiative corrections arise from quantum loops in the boson propagators (Fig. 2.1). The loops create a dependence of the boson masses on parameters such as the top quark mass and the Higgs scalar mass.

Values for the intermediate vector boson (IVB) masses and their sources are given in table 2.3. Using the relations presented above, it is possible to do 


\begin{tabular}{||lr||}
\hline$\Delta M_{Z}$ & $20 \mathrm{MeV}$ \\
$\Delta m_{t}(10 \mathrm{GeV})$ & $50 \mathrm{MeV}$ \\
$\Delta m_{H}(100-1000 \mathrm{GeV})$ & $200 \mathrm{MeV}$ \\
\hline
\end{tabular}

Table 2.4: Uncertainties from various sources for the $\mathrm{W}^{ \pm}$boson mass in the minimal Standard Model.

a consistency check of these masses within the model. Such a test is shown in Fig. 2.2 where the dependence of $M_{W}$ on $M_{Z}$ is shown for various top quark masses. In the figure, one can see that although the $\mathrm{Z}^{0}$ mass is known very precisely, almost no constraint is placed on the $\mathrm{W}^{ \pm}$mass. An extremely precise measurement of the $\mathrm{W}^{ \pm}$would have to be done to constrain the top mass. However, if the top quark mass were determined, this would constrain the $\mathrm{W}^{ \pm}$mass modulo the uncertainty due to the Higgs mass.

Looking at Fig. 2.2, a range of allowed $M_{W}$ can be derived. The source of this mass uncertainty is shown in table 2.4. In this study, it is assumed that the top quark will be found and its mass measured with an error of 10 $\mathrm{GeV}$. Although this leads to a $50 \mathrm{MeV}$ uncertainty, the lack of knowledge of the Higgs mass dominates the error. The total uncertainty of $\approx 200 \mathrm{MeV}$ limits the values of $M_{W}$ that the Standard Model will allow. Hence, better measurements of $M_{W}$ need to be made in order to test the Standard Model.

\subsection{Measuring $M_{W}$}

Measuring the $\mathrm{W}^{ \pm}$mass relies heavily on simulations. This can be seen by examining the kinematics of the boson decay and the subsequent measurement of its decay products. Although this analysis will proceed using the 


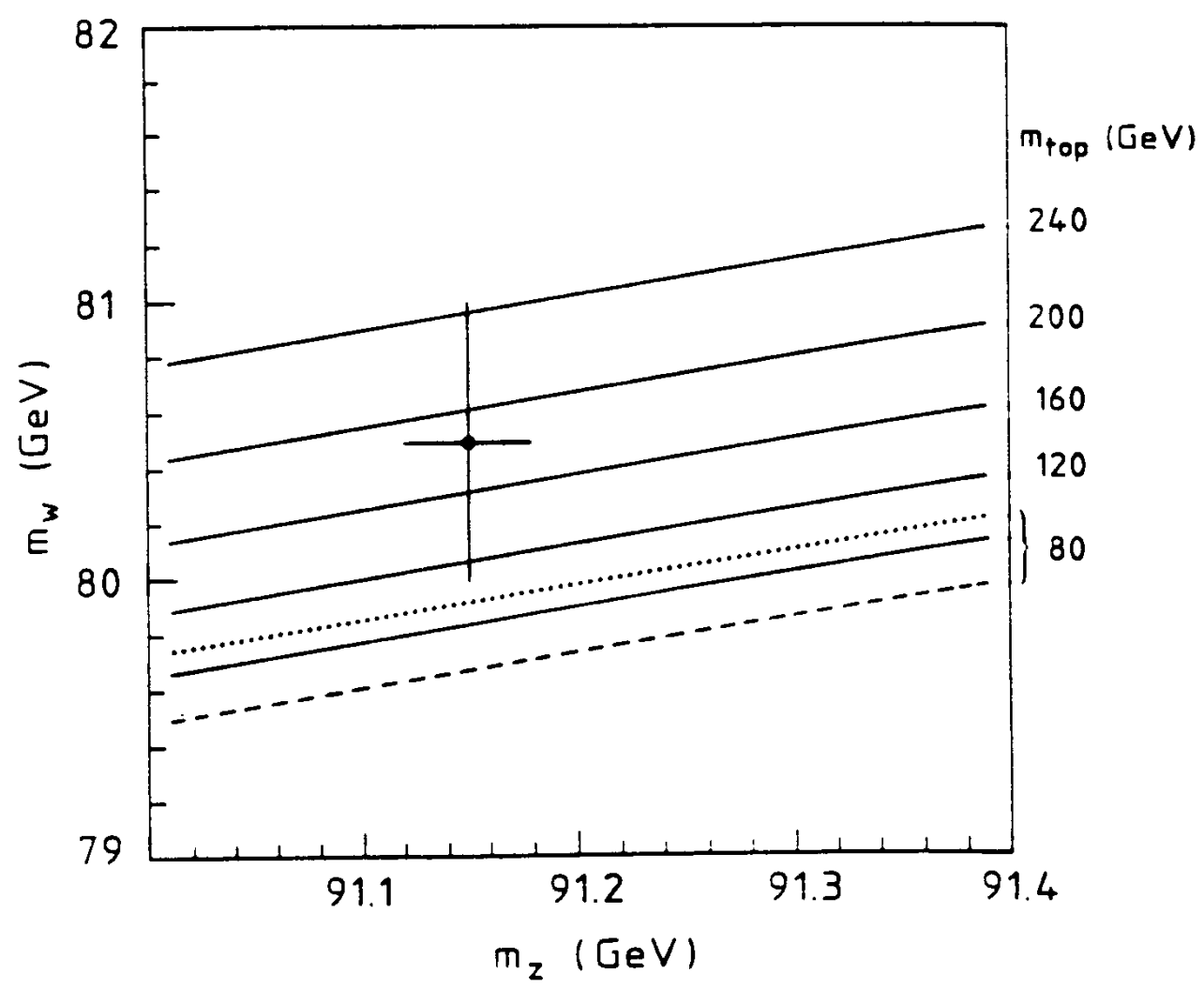

Figure 2.2: Relationship between $M_{Z}$ and $M_{W}$ in the minimal Standard Model for various top masses with $m_{\text {Higgs }}=100 \mathrm{GeV}$. The dotted (dashed) line indicates the prediction for $m_{\text {lop }}=80 \mathrm{GeV}$ with $m_{\text {Higgs }}=10$ (1000) GeV[13]. 


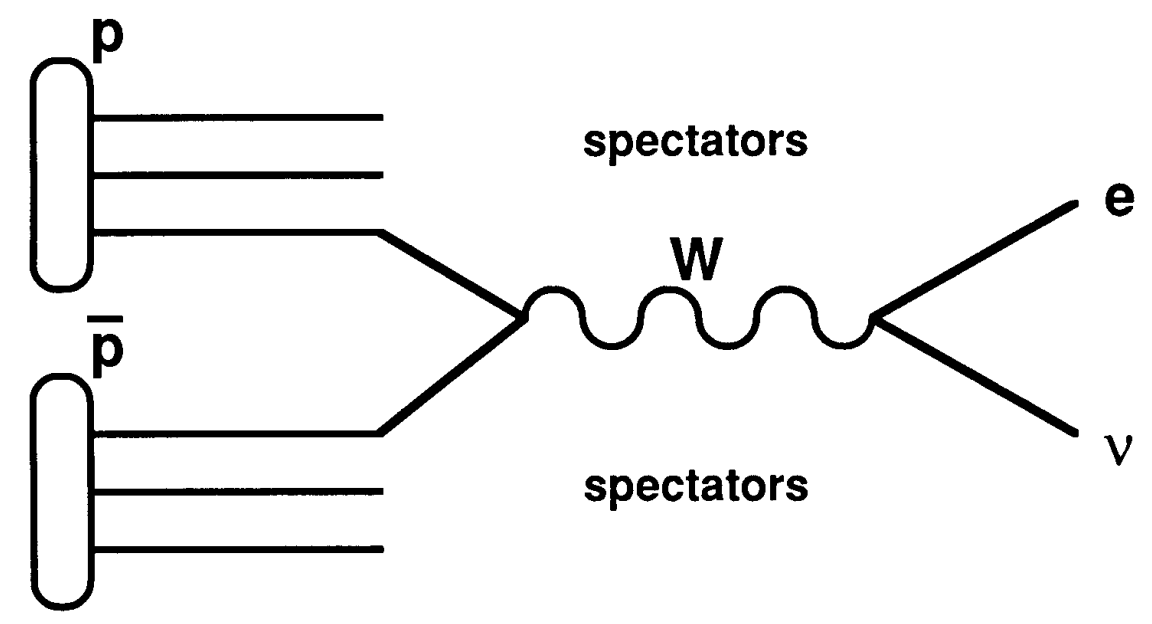

Figure 2.3: Production of the $\mathrm{W}^{ \pm}$boson from $\overline{\mathrm{p}} \mathrm{p}$ collisions and subsequent decay into an electron and neutrino.

electron decay channel due to the superior energy determination possible, the arguments made here also apply to the muon channel. Fig. 2.3 shows the $\mathrm{W}^{ \pm}$ production and decay. Although this is a two body decay with the kinematic parameters well determined, the mass can not be determined as in the case of the $\mathrm{Z}^{0}$ boson where both decay electrons are detected. One of the $\mathrm{W}^{ \pm}$decay products (neutrino) escapes undetected.

To compensate for this lack of knowledge, momentum conservation is employed. Along any axis, the vector sum of the momenta of all particles leaving the collision should add to zero (within the resolution of the detector). Any additional momentum imbalance can be attributed to the undetected particles. 
This is useful for axes transverse to the beamline, but due to detector construction constraints, longitudinal momentum information is lost since many particles escape down the beampipe. We are left with a determination of the transverse components of the neutrino momentum but no information about its longitudinal component.

In the case of the $\mathrm{Z}^{0}$, the mass is determined by fitting the data to an explicit analytical form. The loss of information in $\mathrm{W}^{ \pm}$decays forces a reliance on Monte Carlo generated data to predict the distributions of reconstructed kinematic variables. These simulations generate $\mathrm{W}$ events with the appropriate characteristics, simulate the decay to leptons, and then simulate the detector response to the decay products. Model dependencies in the detector response can result in systematic shifts and uncertainties in the kinematic quantities used to determine the boson mass. We've shown previously that the scale at which these need to be understood is $\approx 200 \mathrm{MeV}$.

The kinematic quantities used for comparison between data and the simulations include the transverse momentum of both the electron and the neutrino. Since the momentum of the electron is measured in the electromagnetic calorimeter, calibration errors in this device can affect the subsequent mass determination. Another quantity used to fit the mass is the two dimensional equivalent of the invariant mass known as the transverse mass:

$$
m_{t}^{2}=2 p_{T}^{e} p_{T}^{\nu}\left(1-\cos \phi^{e \nu}\right)
$$

where:

$$
p_{T}^{l}=\text { leptonic transverse momentum }
$$




$$
\phi^{e \nu}=\text { azimuthal angle between the two leptons }
$$

It turns out that smaller errors result when the transverse mass is used in the mass fit as opposed to the transverse momenta of the leptons separately (Tab. $2.5)[13][14]$.

The details of the fitting procedure are described here. A set of trial $\mathrm{W}^{ \pm}$ masses covering the range of expected masses are chosen. At each mass, an event generator produces a sample of events and decays them into an electron and a neutrino. The decay particles are then passed through a simulation of the detector that reproduces its response characteristics. The response of the detector is then stored and used to produce the kinematic quantities, such as the transverse mass, used to fit the boson mass.

Data accumulated by the detector, passing various trigger requirements and having been reconstructed, are used to produce distributions of the kinematic quantities. A maximum likelihood fit is then performed on the collection of simulated data to arrive at a preferred mass. Interpolations are made between the discrete trial masses to produce a continuous likelihood function and allowing for error analysis.

There are many sources of error in this procedure that can not only increase the final answer's uncertainty, but also shift it. The sources of systematic errors and their magnitudes as determined by the UA2 experiment for this analysis are shown in Tab. 2.5. Included in this list is the uncertainty induced by potential errors in their electron response simulation. This will also be a concern for $\mathrm{D} \emptyset$. This thesis will estimate the systematic biases of 


\begin{tabular}{||lccc||}
\hline Model variation & $m_{t}$ fit & $p_{T}^{e}$ fit & $p_{T}^{\nu}$ fit \\
\hline hadron resolution/response & & & \\
and $p_{T}$ (boson) distribution & \pm 115 & \pm 215 & \pm 350 \\
parton distributions & \pm 100 & \pm 160 & \pm 130 \\
neutrino scale & \pm 85 & - & \pm 170 \\
electron resolution & \pm 40 & \pm 50 & \pm 60 \\
underlying event & \pm 30 & \pm 50 & \pm 20 \\
fit procedure & \pm 100 & \pm 100 & \pm 150 \\
radiative decays & $+40 \pm 40$ & $+60 \pm 60$ & $+160 \pm 160$ \\
\hline total & $+40 \pm 210$ & $+60 \pm 300$ & $+160 \pm 470$ \\
\hline
\end{tabular}

Table 2.5: A summary of the UA2 corrections and systematic errors for each of the $\mathrm{W}$ mass fits. The width of the $\mathrm{W}^{ \pm},{ }_{W}$, is set equal to the Standard Model value of $2.1 \mathrm{GeV}$. All values are in $\mathrm{MeV}$. [13]

using the Monte Carlo simulation developed in our 1991 testbeam experiment in our analysis of the $\mathrm{W}^{ \pm}$mass.

\subsection{Electromagnetic Showers}

When a high energy electromagnetic particle (an electron, positron, or photon) encounters matter, it loses its initial kinetic energy (and rest mass in the case of the positron) through the development of what is known as an electromagnetic shower. Electrons and positrons radiate photons through the bremsstrahlung process. High energy photons create electrons and positrons via pair production. This particle multiplication process continues creating a shower of more and more lower energy particles until the other processes such as ionization loss and the photoelectric effect begin to dominate the propagation of the shower. It is this showering process that is exploited to measure the energy of incident particles in devices known as calorimeters. 
Electrons lose energy by two processes, ionization and radiation. The radiative losses are governed by a material independent unit of length known as the radiation length $\left(X_{0}\right)$. The energy lost by an electron of energy $E$ by radiation in a length $\Delta x$ behaves as $[15]$ :

$$
(\Delta E)_{\text {radiation }}=-E\left(\Delta x / X_{0}\right)
$$

This implies that the average energy of a beam of electrons with initial energy $E_{0}$ after traversing a thickness $x$ will be:

$$
\langle E\rangle=E_{0} e^{-x / X_{0}}
$$

Thus, a radiation length can be defined as the distance in a material that a beam of electrons must pass through to reduce their average energy by a factor e. An expression to determine an approximate value for $X_{0}$ that is accurate to $20 \%$ for materials with atomic mass $\mathrm{A}$ and atomic numbers $\mathrm{Z}$ from 13 to 92 is $[15]$ :

$$
X_{0}=180 \frac{A}{Z^{2}} \frac{\mathrm{g}}{\mathrm{cm}^{2}}
$$

The ionization losses are governed by the Bethe-Bloch formula[16]:

$$
-\left(\frac{d E}{d x}\right)=4 \pi N_{A} r_{e}^{2} m_{e} c^{2} z^{2} \frac{Z}{A} \frac{1}{\beta^{2}}\left[\ln \left(\frac{2 m_{e} c^{2} \gamma^{2} \beta^{2}}{I}\right)-\beta^{2}-\frac{\delta}{2}\right]
$$

where the incident particle has charge $z e, N_{A}$ is Avogadro's number, $m_{e}$ is the electron mass, $r_{e}$ is the classical electron radius, $\beta=v / c, \gamma=\left(1-\beta^{2}\right)^{-\frac{1}{2}}$, and $x$ is the path length in the medium measured in $\mathrm{g} / \mathrm{cm}^{2} . I$ is an effective ionization potential with $I \simeq 16 Z^{0.9} \mathrm{eV}$ (for $Z>1$ ). At nonrelativistic velocities, $d E / d x$ varies as $1 / \beta^{2}$ reaching a minimum for $E \sim 3 M c^{2}$ ( $M$ is the mass of the 
incident particle). For greater incident energies, the energy loss experiences what is known as a relativistic rise. This increase varies logarithmically with incident energy and is caused by the relativistic intensification of the transverse electric fields which increases the effects of distant collisions. The screening of the enhanced transverse electric field by atomic electrons is represented by $\delta$. For energetic particles, $\delta$ approaches $2 \ln \gamma$ plus a constant term. This causes the bracketed portion of Eq. 2.6 to increase asymptotically as $\ln \gamma$ instead of $2 \ln \gamma[16]$.

Another important parameter affecting electromagnetic showers is known as the critical energy $\left(\epsilon_{0}\right)$. This is approximately the energy at which ionization losses become as important as radiation losses. An expression giving the approximate value for the critical energy is[17]:

$$
\epsilon_{0} \simeq \frac{550}{Z}(\mathrm{MeV})
$$

This number is highly material dependent, ranging from $95 \mathrm{MeV}$ for carbon to $9 \mathrm{MeV}$ for uranium (Fig. 2.4).

To illustrate the development of an electromagnetic shower, consider the following simple model. We assume that all particles $\left(e^{ \pm}, \gamma\right)$ interact after $1 X_{0}$ imparting equal amounts of energy to their secondaries. Additionally, charged particles deposit a constant amount of energy per unit length through ionization independent of energy until the energy of the particle drops below the critical energy, $\epsilon_{0}$, wherein it deposits all of its energy locally through collisions. Following an electron of energy $E_{0}$ which is incident on some material, it travels $1 X_{0}$ before it emits a bremsstrahlung photon. The electron 


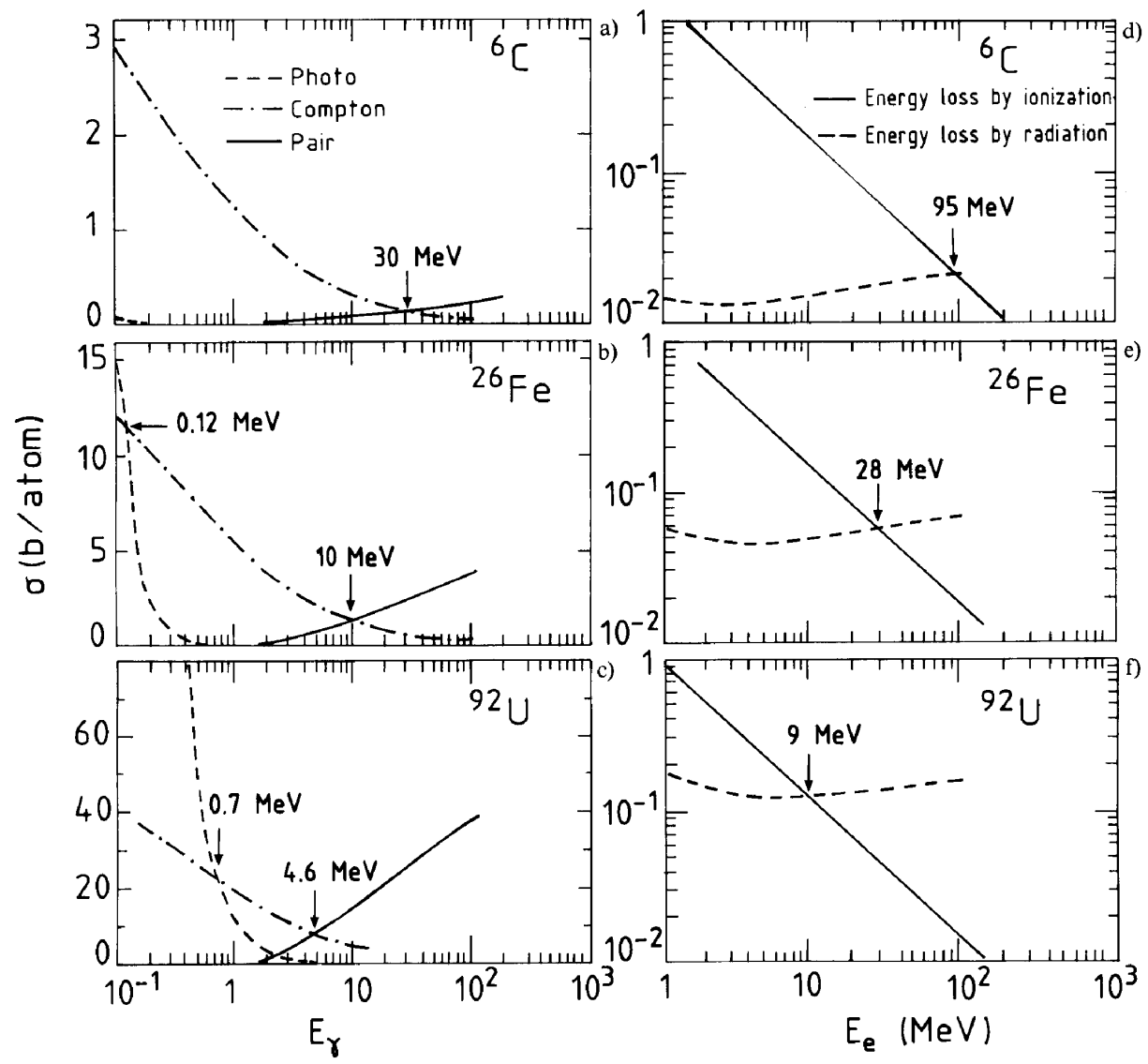

Figure 2.4: Cross sections for various processes in carbon, iron, and uranium as a function of particle energy for both photons $\left(E_{\gamma}\right)$ and electrons $\left(E_{e}\right)[18]$. The critical energy for each material is shown on the electron cross section plot. 


\begin{tabular}{||cccc||}
\hline Depth $\left(X_{0}\right)$ & Number of Particles & Energy & Particles \\
\hline 0 & 1 & $E_{0}$ & $e^{-}$ \\
1 & 2 & $\frac{E_{0}}{2}+\frac{E_{0}}{2}$ & $e^{-}+\gamma$ \\
2 & 4 & $\frac{E_{0}}{4}+\frac{E_{0}}{4}+\frac{E_{0}}{4}+\frac{E_{0}}{4}$ & $e^{-}+\gamma+e^{-} e^{+}$ \\
$\vdots$ & $\vdots$ & $\vdots$ & \\
$t$ & $2^{t}$ & $E_{0} 2^{-t}$ & \\
\hline
\end{tabular}

Table 2.6: Electromagnetic shower development showing the number and type of particles as well as the average energy as a function of depth in the shower[19].

and photon will each have an energy of $E_{0} / 2$. After another radiation length, the electron will again radiate a photon while the first photon will create an electron-positron pair. Each of these four particles will posses an energy $E_{0} / 4$. This is illustrated in Tab. 2.6. The process continues with the number of particles at depth $\mathrm{t}\left(\right.$ in $\left.X_{0}\right)$ given by:

$$
N_{e, \gamma}=2^{t}
$$

with the average energy per particle at this depth given by:

$$
\epsilon=E_{0} 2^{-t}
$$

When the average energy reaches $\epsilon_{0}$, the critical energy, the development ceases and the shower dissipates. The depth at which this occurs is:

$$
\begin{aligned}
\epsilon_{0} & =E_{0} 2^{-t_{\max }} \\
t_{\text {max }} & =\frac{\ln \frac{E}{\epsilon_{0}}}{\ln 2}
\end{aligned}
$$

Integrating over the whole shower, the total number of charged particles is:

$$
N_{c}=\frac{2}{3} \int_{0}^{t_{\max }} N(t) d t
$$




$$
\begin{aligned}
& \simeq \frac{2}{3} \frac{e^{t_{\text {max }} \ln 2}}{\ln 2} \\
& =\frac{2}{3} \frac{E}{\epsilon_{0} \ln 2}
\end{aligned}
$$

The factor of $2 / 3$ is due to the fact that electrons, positrons, and photons are produced in equal numbers at energies above $\epsilon_{0}$.

This number, $N_{c}$, is also equal to the average total charged track length $\langle T\rangle$ in radiation lengths:

$$
\langle T\rangle=N_{c} X_{0} \simeq \frac{E}{\epsilon_{0}} X_{0}
$$

This shows that the average total charged track length is proportional to the total incident energy. This is what allows calorimeters to work. In a real calorimeter, the average detected charged track length, $\left\langle T_{d}\right\rangle$, is always less than $\langle T\rangle$ :

$$
\left\langle T_{d}\right\rangle \simeq F(\xi) \frac{E}{\epsilon_{0}} X_{0}
$$

where $F(\xi)<1$ since all detectors have some energy $\xi$ below which they are not sensitive to the charged electrons and positrons[19].

So far we have only dealt with the average behavior of showers. The energy measurement resolution is determined by the fluctuations about this average behavior. The intrinsic energy measurement resolution of an electromagnetic shower depends on the elementary processes that govern the shower. For a fully contained shower, these fluctuations are just those of the total charge track length $T$. For nonzero $\xi$ 's, the detected track length is less than the total track length so that the fluctuations in $T$ form a lower bound:

$$
\frac{\sigma(E)}{E} \geq \frac{\sigma(T)}{T}
$$




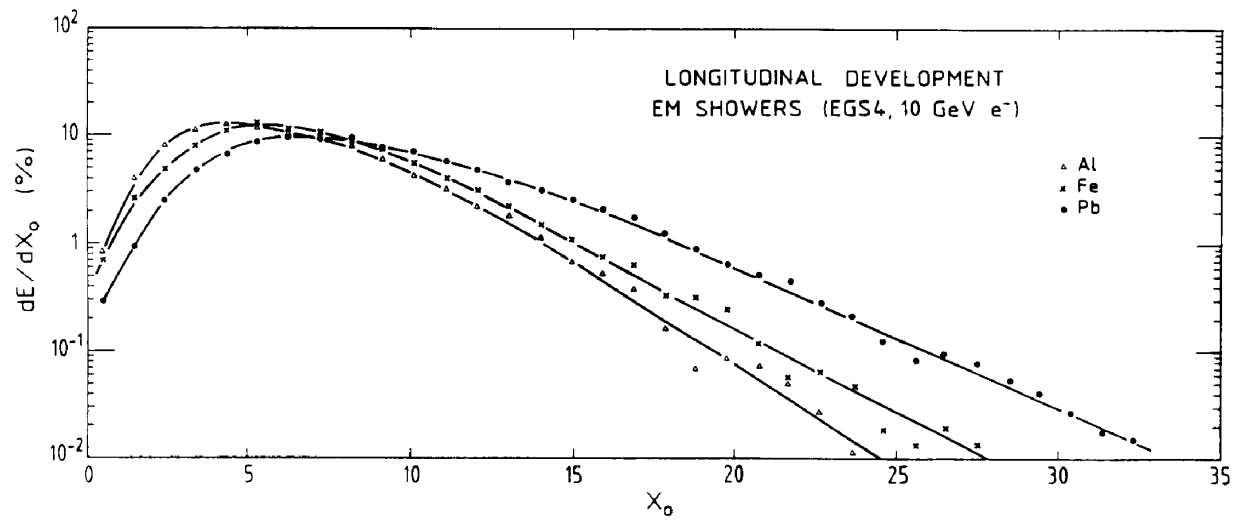

Figure 2.5: The electromagnetic shower development as a function of depth for various materials using the EGS4 Monte Carlo showing the approximate scaling of the longitudinal profile when expressed in units of radiation lengths[18].

The processes involved in producing the secondary particles are statistical in nature and hence the fluctuations in $T$ vary as $\sqrt{T}$. From this we have:

$$
\frac{\sigma(E)}{E} \propto \frac{1}{\sqrt{T}} \propto \frac{1}{\sqrt{E}}
$$

This gives a lower bound on the resolution since leakage and detector inefficiencies will further degrade the resolution.

The longitudinal structure of the shower scales in units of $X_{0}$. When expressed in these units, the behavior of the shower is approximately material independent (Fig. 2.5).

The longitudinal deposition of energy can be expressed as[15]:

$$
\frac{d E}{d t}=E_{o} \frac{b^{\rho+1}}{,(\rho+1)} t^{\rho} e^{-b t}
$$

where $\mathrm{t}$ is in units of $X_{0}, b$ in units of $X_{0}^{-1}$, and $\rho$ is a dimensionless. It turns out that $\rho$ and $b$ are energy dependent parameters, but that $b \sim 0.5$ 
and $t_{\text {max }}=\rho / b\left(t_{\text {max }}\right.$ is the location of the maximum of Eq. 2.16) [17]. This formula represents the average longitudinal energy deposition. Transversely, the shower can be characterized by a central core with a surrounding halo. The lateral spread of the central core is due to the multiple scattering of the shower particles and can be described by a cylinder of radius $R[17]$ :

$$
\begin{aligned}
R & \approx 2 \rho_{M} \\
\rho_{M} & =21 \frac{X_{0}}{\epsilon_{0}} \\
& \simeq 7 \frac{A}{Z} \mathrm{~g} / \mathrm{cm}^{2}
\end{aligned}
$$

where $\rho_{M}$ is known as the Moliére radius. The dominant process responsible for the shower spreading beyond the central core is the relatively long distance propagation of low energy photons. This is governed by the minimum in the photon attenuation coefficient which occurs around $1-10 \mathrm{MeV}$ for lead and uranium but generally higher energies for lighter elements (Fig. 2.6)[18].

\subsection{Calorimetry}

A calorimeter, as its name suggests, is a device wherein an incident particle converts its kinetic energy, and sometimes its mass, into alternative forms of energy more readily measurable, e.g. light, heat, or ionization. Two types of calorimeters exist: homogeneous and sampling. Homogeneous calorimeters consist of blocks of matter all of which are sensitive to the signal of the incident particle. All of the particle's energy is readout by the calorimeter. Sampling calorimeters, however, consist of alternating layers of passive and active ma- 


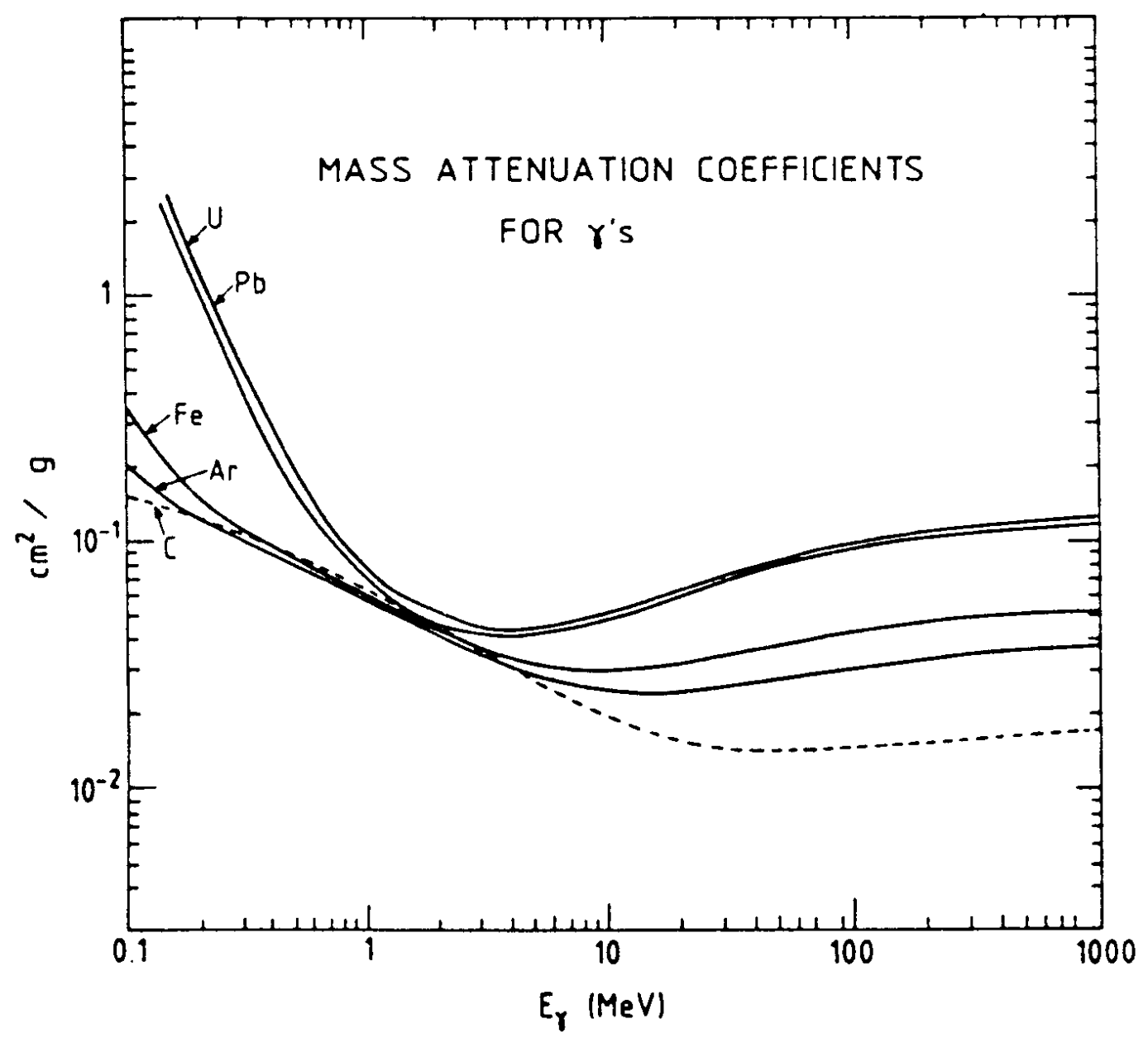

Figure 2.6: The mass attenuation coefficients for photons in different materials as a function of energy $\left(E_{\gamma}\right)[18]$. 
terial and only the energy lost by the incident particle in the active layers is seen. In most practical cases, this visible energy is actually a small fraction of the total $(<20 \%)$.

For hadron collider environments, homogeneous calorimeters are impractical due to the large amounts of material necessary to totally contain a shower. In these environments, the advantages of sampling calorimeters become evident. By making use of relatively dense absorber material interspersed between the active media, a relatively compact and cost effective device can be constructed.

Earlier we discussed the concept of total track length of the shower as being proportional to the total energy of the shower. In the case of a sampling calorimeter, only a fraction of the tracks cross the active planes. Hence, a correction must be applied to the raw signal to arrive at the total energy. This is known as a sampling fraction correction. Simply, it can be written as:

$$
\text { SamplingFraction }=\frac{E_{\text {live }}}{E_{\text {live }}+E_{\text {dead }}}
$$

where the energies are calculated for minimum ionizing particles.

Another feature of sampling calorimeters is an additional source of energy measurement error. This is due to fluctuations in the number of charged tracks crossing the active planes. As it turns out, these fluctuations dominate the intrinsic fluctuations of the shower we discussed before. Whereas the intrinsic fluctuations contributed $<1 \% / \sqrt{E}(E$ in $\mathrm{GeV})$ to the energy uncertainty, the shower fluctuations can contribute as much as $10-20 / \% \sqrt{E}(E$ in $\mathrm{GeV})$.

Another contributor to the overall energy resolution is leakage, both trans- 
verse and longitudinal. This is because with leakage the detector is no longer sampling the entire shower which will lead to low end tails and a nongaussian energy distribution. The detector should be designed to be thick enough to contain the entire shower. The effect on the energy resolution as a result of incomplete longitudinal containment can be parameterized by [17]:

$$
\sigma / E \simeq[\sigma / E]_{f=0} \times[1+2 \sqrt{E(G e V)} \times f]
$$

where $f$ is the fraction of the incident energy escaping out of the back of the calorimeter and $[\sigma / E]_{f=0}$ is the ideal resolution with no leakage. It also turns out that longitudinal leakage is more serious than lateral leakage since the fluctuations about the average longitudinal loss are larger than those about the average transverse loss (Fig. 2.7) [17].

In the arguments to this point, we have assumed that the energy deposited by a charged particle in an electromagnetic shower was equivalent to that of a minimum ionizing particle (mip). Most electrons and positrons in the shower have energies exceeding a few $\mathrm{MeV}$ (the energy at which an electron is minimum ionizing). Therefore, the relativistic particles will deposit more energy than the minimum ionizing value due to the relativistic rise. Although the above assumption is flawed, the important physical characteristics of an electromagnetic shower are introduced in a calculable way by this model.

The previous discussion concerning total track length assumed that the individual shower particles could be replaced by the same number of mip's having the same total track length. This is not the case with a sampling calorimeter. Since a sampling calorimeter is composed of different media with 


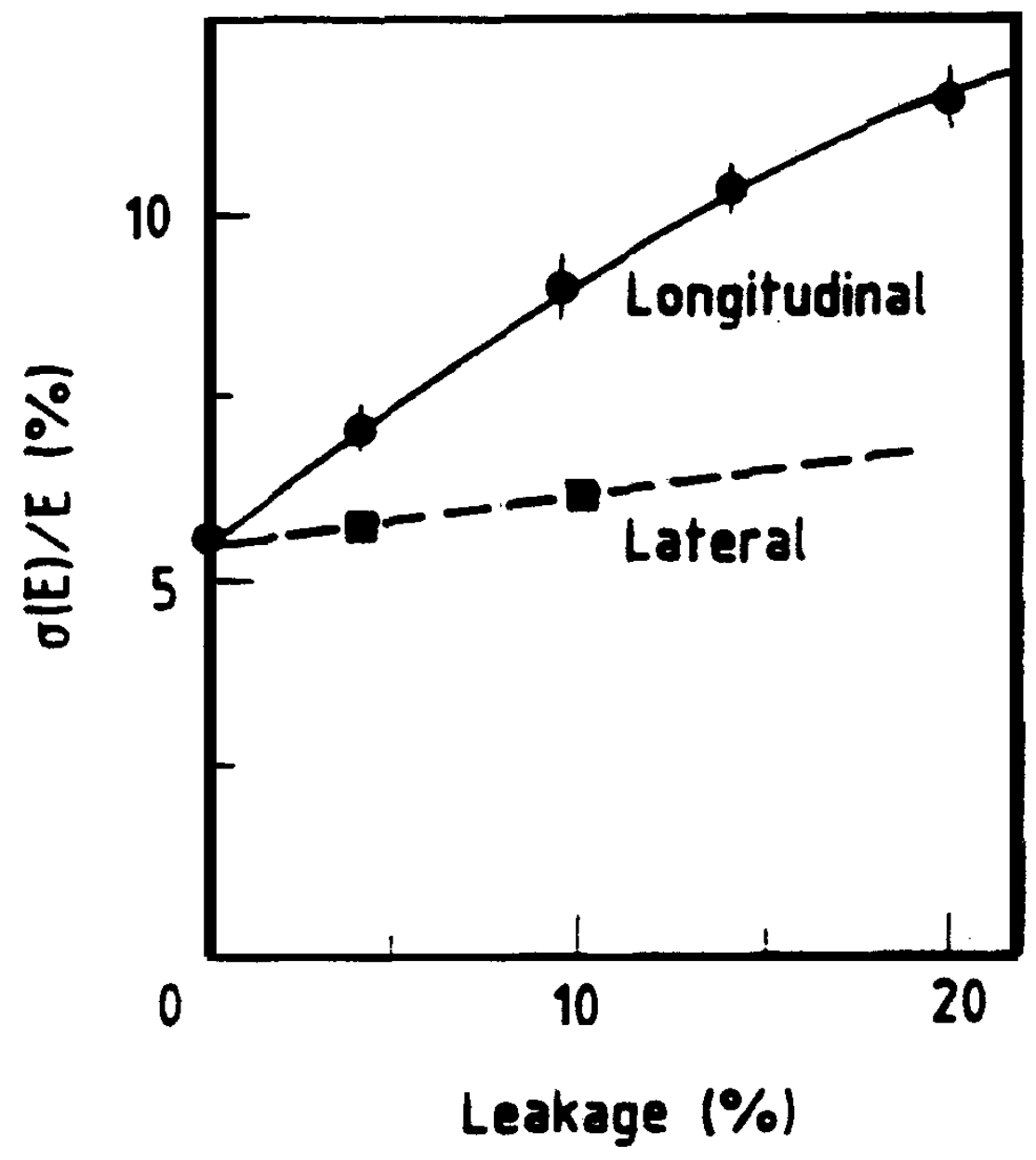

Figure 2.7: The effects of both longitudinal and transverse leakage on the energy resolution as measured for electrons in the CHARM neutrino calorimeter[17]. 
different physical properties, it can not be assumed that processes that occur at the low energy end of the shower will be identical in both the passive and active planes. At high energies, bremsstrahlung and pair creation will dominate in all materials. However, the onset of the shower dissipation and the relative importance of the low energy processes can be markedly different between the different materials. Geometrical considerations can also play a role in determining the response.

As an example, consider a uranium/liquid argon calorimeter. Again, the high energy part of the shower should be more or less material independent scaling as $X_{o}$. However, $\epsilon_{0}$ which determines the point at which the shower stops growing and begins to dissipate is quite different for these two materials $\left(\epsilon_{U} \approx 10 \mathrm{MeV}, \epsilon_{A r} \approx 40 \mathrm{MeV}\right)$.

Hence, in a sampling calorimeter, the differing cross sections for various processes in the active and passive media will result in the detected energy being different from minimum ionizing. Initially, during the high energy development of the shower, the bremsstralung $\left(\propto Z^{2}\right)$ and pair production $\left(\propto Z^{2}\right)$ processes dominate. However, at the lower energies typical of the later stages of the shower, ionization $(\propto Z)$, Compton scattering $(\propto Z)$, and the photoelectric effect $\left(\propto Z^{5}\right)$ dominate. The strong $Z$ dependence of the photo-electric cross sections can lead to a disproportionate fraction of the energy being deposited in the high $\mathrm{Z}$ material. More photons will liberate photo-electrons in the high $Z$ material which then deposit their energy locally[19]. This effect is illustrated in Fig. 2.8 where the $e /$ mip ratio is shown for liquid argon and scintillator calorimeters as a function of the $Z$ of the absorber medium. If the 


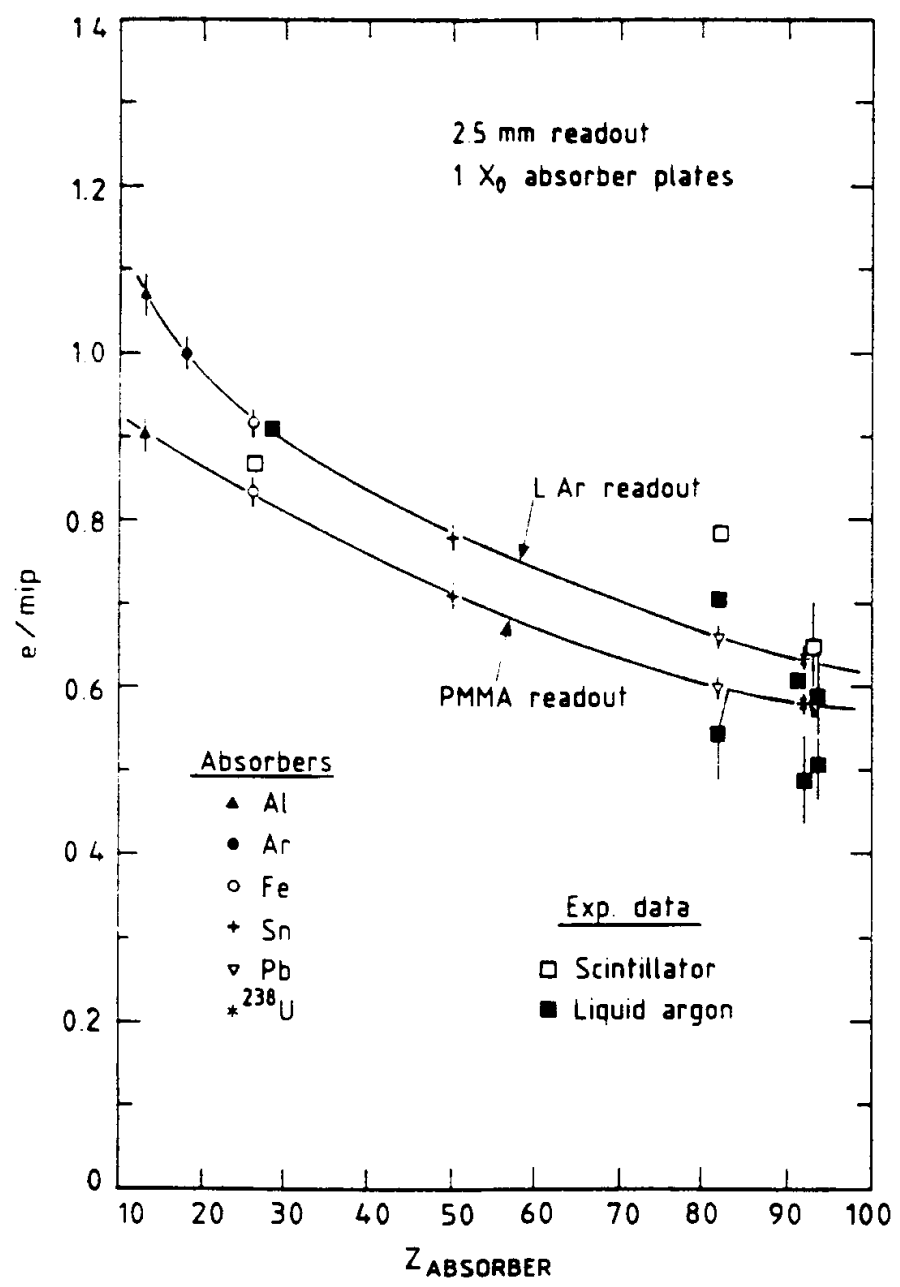

Figure 2.8: The e/mip ratio for liquid argon and scintillator (PMMA) calorimeters as a function of the $\mathrm{Z}$ of the absorbing medium. The absorber layers are $1 X_{0}$ thick. Results from EGS4 Monte Carlo simulation[18]. 
absorber plates are thin enough, however, some of the photo-electrons may find there way to the active medium[19]. This is shown in Fig. 2.9 where the e/mip ratio is shown as a function of absorber plate thickness. For uranium, this effect saturates for plates greater than $3 \mathrm{~mm}$ thick. 


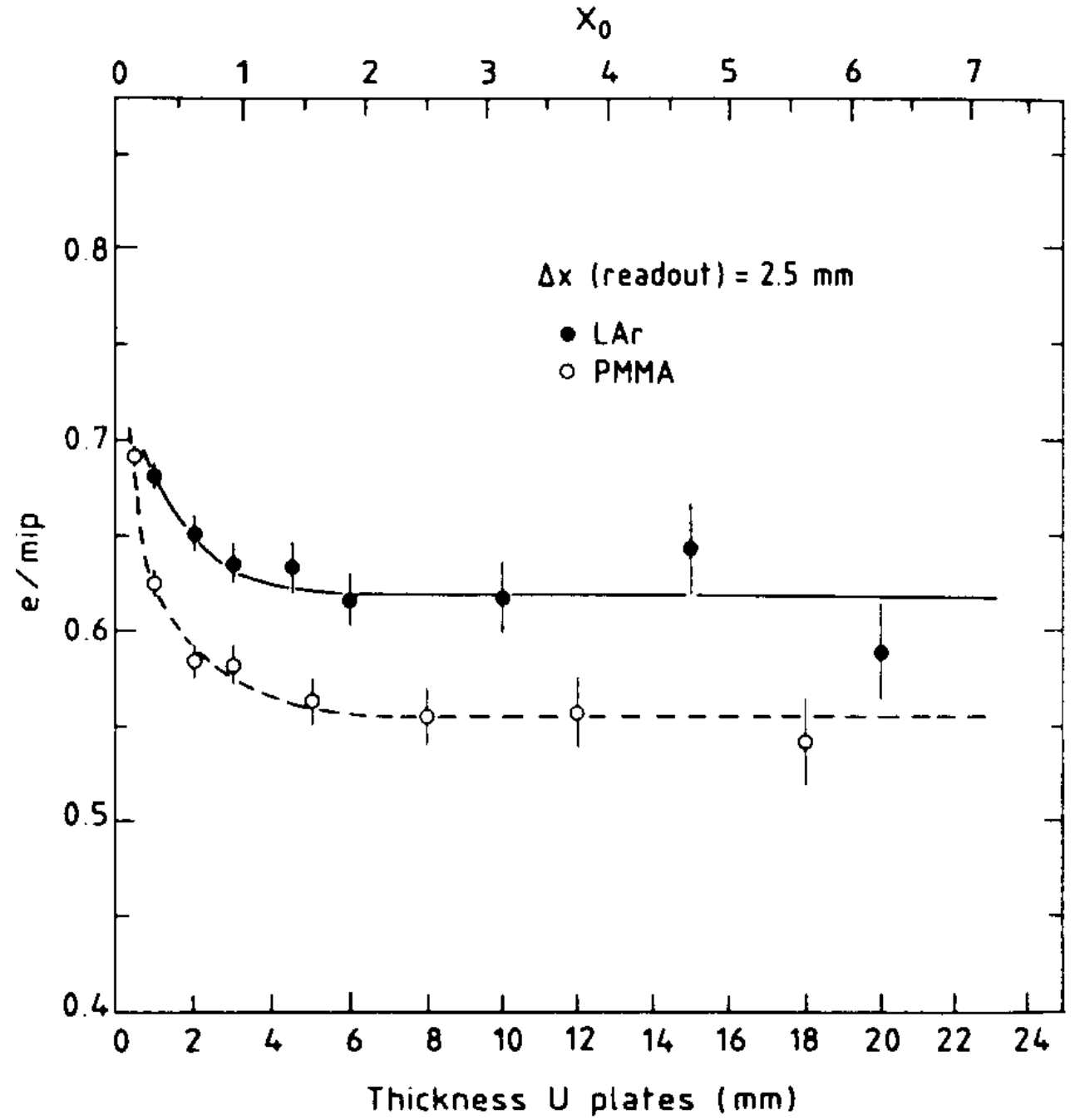

Figure 2.9: The e/mip ratio for liquid argon and scintillator (PMMA) calorimeters for various uranium absorber plate thicknesses[18]. 


\section{Chapter 3}

\section{The DØ Detector}

\subsection{Detector Overview}

The DØ Detector is one of two collider detectors currently operating at the Fermilab Tevatron. It has been designed to study the physics available from $\bar{p} p$ collisions with a center of mass energy of $1.8 \mathrm{TeV}$. To accomplish its goals, the detector emphasizes efficient lepton identification and high resolution energy measurement of leptons, jets, and missing energy.

The detector is composed of three major systems: central tracking, calorimetry, and muon. A picture of the detector is shown in Fig. 3.1. The coordinate system used defines the $\mathrm{z}$ axis along the direction of the proton beam, the $\mathrm{y}$ axis points $\mathrm{up}$, and the $\mathrm{x}$ axis points radially outward from the center of the Tevatron ring. The angular coordinates $\theta$ and $\phi$ are the usual polar and azimuthal angles. Pseudorapidity, $\eta=-\ln \tan (\theta / 2)$, is a measure of the polar angle appropriate for longitudinal phase space.

$\mathrm{D} \emptyset$ does not possess a central magnetic field and relies on energy mea- 


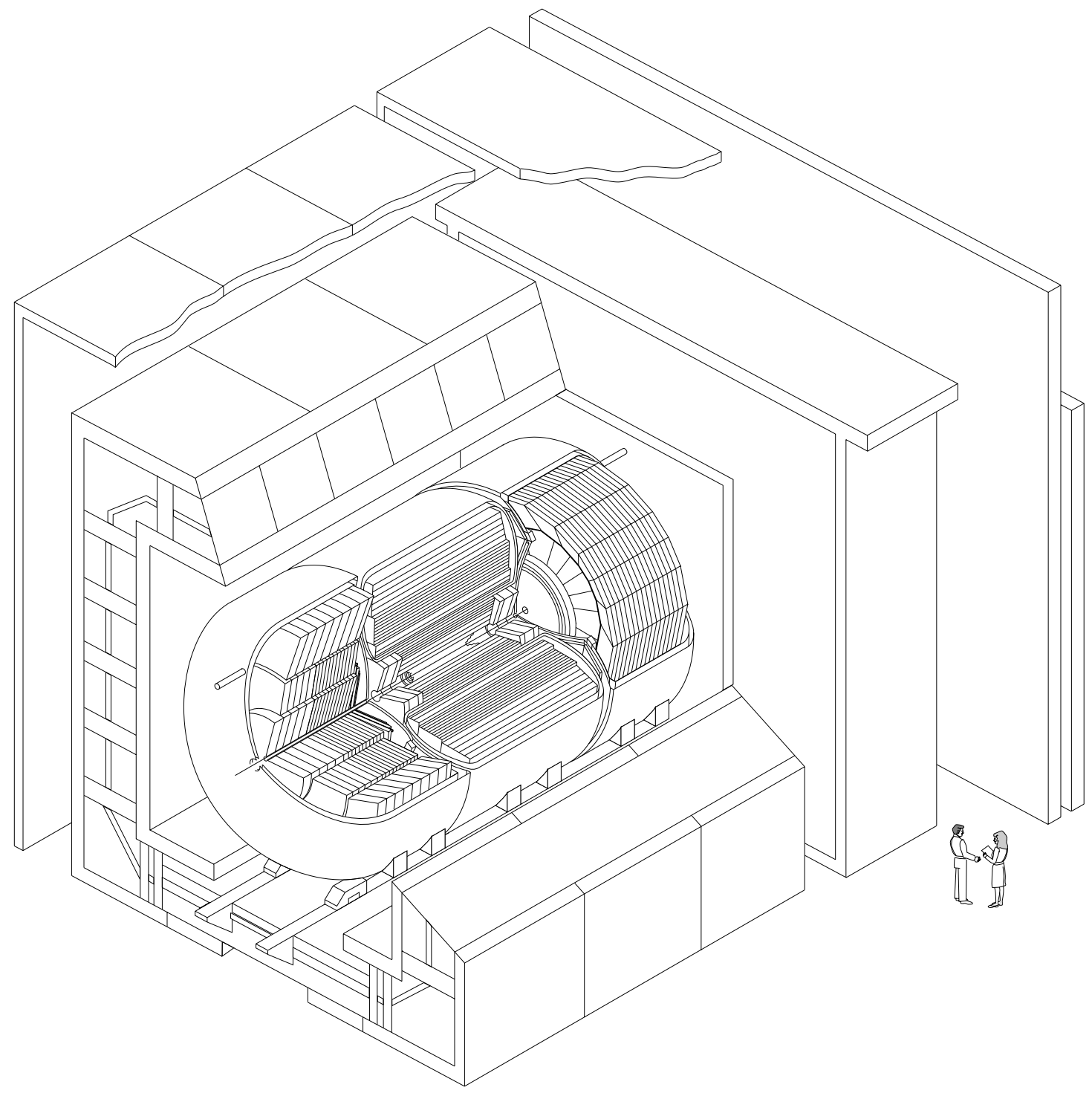

D $\emptyset$ Detector

Figure 3.1: The DØ Detector. 
surements from the calorimeter rather than momentum measurements made by the central tracking system. The primary purpose of the central tracking system is charged particle tracking and identification. Information from this system is used to identify photons and other neutrals that may mimic an electron signal in the calorimeter as well as to locate the primary vertex of the event. The central tracking system provides charged particle tracking out to a pseudorapidity of $\sim 4$.

The vertex detector (VTX) is the innermost chamber. It provides tracking out to a pseudorapidity of 1.0. With a single track resolution of $\sim 50 \mu \mathrm{m}$ in the $\mathrm{r}-\phi$ plane, this detector aids in the rejection of photons that convert before the next layer of tracking, the transition radiation detector (TRD) [20]. The TRD relies on the transition radiation emitted by charged particles as they traverse materials with differing dielectric properties. Electrons produce a unique signature that differentiates them from other charged particles. The TRD provides coverage out to a pseudorapidity of $\sim 1.0$.

Another set of tracking chambers surrounds the VTX and the TRD. Out to a pseudorapidity of 1.0 , tracking in this region is accomplished by the central drift chamber (CDC). Beyond this pseudorapidity, the tracking is done by the forward drift chambers (FDC). These chambers are designed to have resolutions of $\sim 200 \mu \mathrm{m}$ in the $\mathrm{r}-\phi$ plane and $>2 \mathrm{~mm}$ along the $\mathrm{z}$ axis for single isolated tracks[20]. The tracks from these chambers are matched to energy depositions in the calorimeter as an aid in particle identification. These tracks are also used in primary vertex determination.

The decision to forego a central magnetic field is based on measurement 
resolutions. Typical fractional momentum measurement resolutions of wire chambers embedded in a magnetic field scale with the momentum:

$$
\frac{\delta p}{p} \propto p
$$

with a proportionality constant $\sim 0.1 \%$. Calorimeter fractional energy measurement resolutions scale as the inverse of the square root of the energy:

$$
\frac{\delta E}{E} \propto \frac{1}{\sqrt{E}}
$$

with the proportionality constant on the order of $15 \%$. For a $50 \mathrm{GeV}$ electron, the calorimeter measurement is superior to the tracking chamber measurement $(\delta p / p \sim 5 \%, \delta E / E \sim 2 \%)$

The above considerations show that the energy resolution of the electromagnetic calorimeter is of importance in the $D \emptyset$ detector. A finely segmented readout system is also important. This provides the shower shape information useful for position measurements and particle identification. For these reasons, a sampling calorimeter was designed with uranium as the passive medium and liquid argon as the active medium. Together, they provide energy resolutions for electromagnetic showers that should scale as $15 \% / \sqrt{E}(E$ in $\mathrm{GeV})$. The finely segmented readout possible with liquid argon calorimetry provides position resolution for electromagnetic showers of $\sim 2 \mathrm{~mm}$ and shower shape information, both longitudinal and transverse to the shower axis, for background rejection. These measurements are provided by the central electromagnetic calorimeter $(|\eta|<1.2)$ and the endcap electromagnetic calorimeter $(1.4<|\eta|<4.0)[20]$. 
The muon system consists of five magnetized iron toroids surrounding the calorimeter. The primary purpose is to measure the momentum of muons emanating from the interaction region. Position measurements are made before and after the magnet providing an entering and exiting track. From this, a momentum is determined. Cosmic rays are rejected using a scintillator shield located on top of the whole detector. Hadronic punchthrough is minimized by the large amount of material between the interaction region and the muon system. Muon coverage is hermetic extending out to $|\eta|<3.8[20]$.

\subsection{Uranium/Liquid Argon Calorimeters}

At the heart of $D \emptyset$ is the uranium/liquid argon calorimeter. A picture of the calorimeter is shown in Fig. 3.2. The calorimeter design was driven by the desire to build a highly capable, compact detector optimized for the study of high $p_{T}$ physics topics of interest at the Fermilab Tevatron. High priority was given to electron identification and measurement. Hermeticity was also important to correctly infer the presence of non-interacting particles and measure their energies. This was accomplished by implementing the same technology and design throughout the entire calorimeter.

\subsubsection{General Design}

To avoid the problems associated with calibrating calorimeters based on differing designs, the $\mathrm{D} \emptyset$ calorimeter makes use of the same technology over all of its sensitive coverage. With the exception of the tail catching section of the 


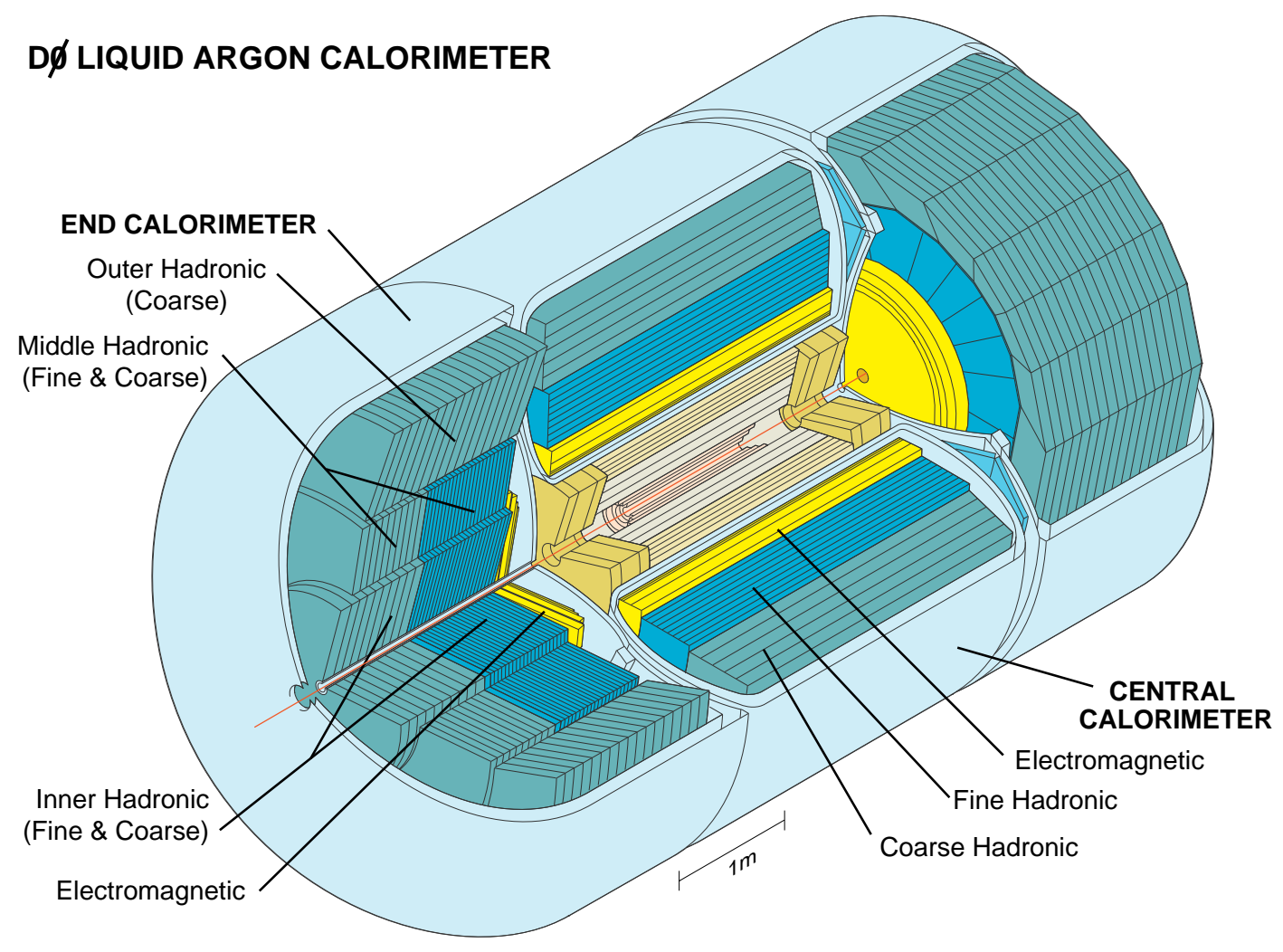

Figure 3.2: Cutaway View of the DØ Calorimeters 


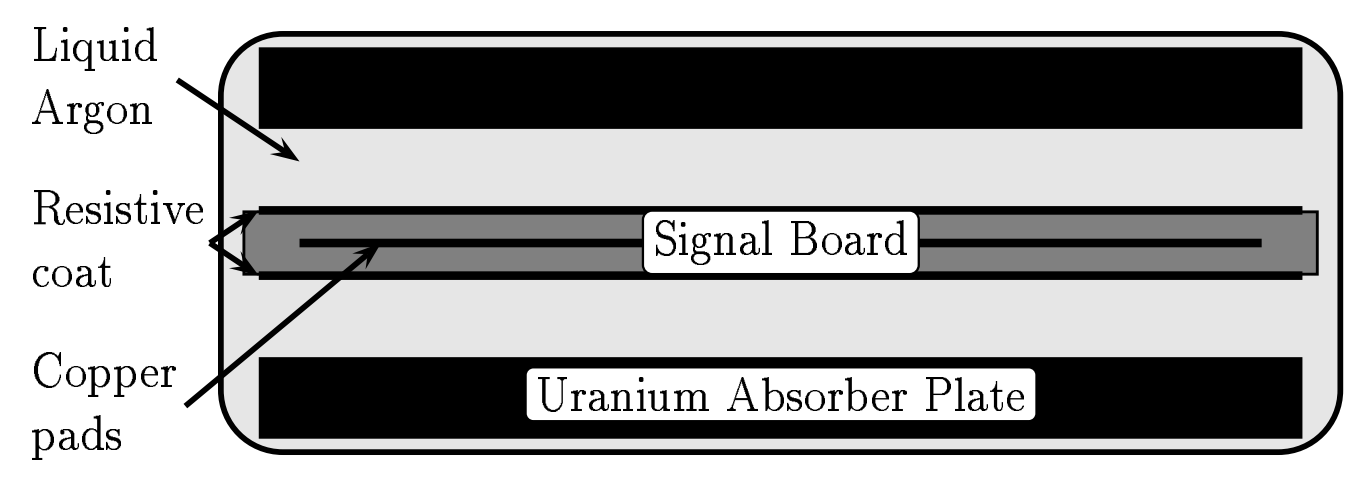

Figure 3.3: The standard unit cell used in the DØ central calorimeter.

hadronic calorimeters that make use of copper or stainless steel, uranium and uranium alloys are used as the absorbing material. Uranium's high density allows for a compact design.

The basic unit cell design is shown in Fig. 3.3. The cell consists of alternating layers of absorber and liquid argon. Located between absorber plates, separating the two $2.3 \mathrm{~mm}$ liquid argon gaps, is a signal collection board. It is constructed from two G-10 (an epoxy/fiberglass composite) boards laminated together, one of which has the pad structure etched out on its copper cladding. The board is held in place in the gap by delrin spacers, passing through the board, extending $2.3 \mathrm{~mm}$ on one side and $1.5 \mathrm{~mm}$ on the other, with the long/short orientation alternating on adjacent spacers. This maintains the standard liquid argon gap while allowing for some freedom of motion necessary during the detector cooldown. A resistive epoxy coating is placed on the exterior surfaces of the board. High voltage $(2.0-2.5 \mathrm{kV})$ is applied to this coating while the absorber plates are kept at ground potential. This 


\begin{tabular}{||l|ccc||}
\hline & CCEM & CCFH & CCCH \\
\hline Inner radius & 84.1 & 105.8 & 170.9 \\
Outer radius & 104.5 & 166.2 & 219.3 \\
Number of modules & 32 & 16 & 16 \\
Absorber & $0.3 \mathrm{~cm} \mathrm{U}$ & $0.6 \mathrm{~cm} \mathrm{U}$ & $4.65 \mathrm{~cm} \mathrm{Cu}$ \\
Endplate thickness & 1.9 & 3.2 & 4.4 \\
Sideskin thickness & .17 & .27 & .27 \\
Frontplate thickness & .125 & .48 & .48 \\
\hline
\end{tabular}

Table 3.1: Design parameters for the central calorimeter modules. All length units are centimeters.

establishes the electric field responsible for the electron drift in the gap.

\subsubsection{The Central Calorimeter}

The central calorimeter $(\mathrm{CC})$ is a barrel shaped detector whose central axis is the Tevatron beamline. Housed in its own double walled cryostat to contain the liquid argon, the CC is composed of three different types of modules: electromagnetic (CCEM), fine hadronic (CCFH), and coarse hadronic (CCCH). The design parameters of the $\mathrm{CC}$ modules are given in Tab. 3.1. These modules are joined together to form three concentric cylinders. Some design concepts are shared by the different module types. The innards of the module are contained within a stainless steel box. The box consists of a front (inner radius) and back (outer radius) plate joined to sideskins, forming the boundary between modules, and endplates. The endplates, load bearing plates located at either face of the cylinder, support the weight of each module and serve as an attachment point for fasteners holding the modules to one another.

The electromagnetic calorimeter forms the innermost ring. The absorber 


\begin{tabular}{||l|ccc||}
\hline & CCEM & CCFH & CCCH \\
\cline { 1 - 1 } $\begin{array}{l}\text { Transverse } \\
\text { segmentation } \\
(\Delta \eta \times \Delta \phi)\end{array}$ & $0.1 \times 0.1^{a}$ & $0.1 \times 0.1$ & $0.1 \times 0.1$ \\
\cline { 1 - 1 } $\begin{array}{l}\text { Longitudinal } \\
\text { segmentation }\end{array}$ & 4 & 3 & 1 \\
\cline { 1 - 1 } $\begin{array}{l}\text { Radiation } \\
\text { lengths }\left(X_{0}\right)\end{array}$ & 20.5 & $\sim 96$ & $\sim 30$ \\
\cline { 1 - 1 } $\begin{array}{l}\text { Absorbtion } \\
\text { lengths }\left(\lambda_{0}\right)\end{array}$ & 0.76 & 3.2 & 3.2 \\
\hline
\end{tabular}

Table 3.2: Physics design parameters of the central calorimeter modules.

${ }^{a} 0.05 \times 0.05$ in the third layer

used is depleted uranium. The CCEM contains 21 unit cells, each cell comprising $\sim 1$ radiation length, combining to make the module 20.5 radiation lengths thick (Tab. 3.2). The module is highly segmented both longitudinally and transversely. The unit cells are grouped into four individually readout layers. The first layer consists of two cells, the second has two cells, the third has seven cells, while the fourth has the remaining ten. The longitudinal information aids in background rejection. A schematic of the signal board pad design is shown in Fig. 3.4. Transversely, the pads are $0.1 \times 0.1$ in pseudorapidity by azimuthal angle. In addition, the third layer is further segmented to $0.05 \times$ 0.05. This allows for electromagnetic shower position resolution of $2 \mathrm{~mm}[20]$. While full azimuthal coverage is provided, the pseudorapidity coverage extends out to 1.2 (Fig. 3.5).

The outer rings of the CC compose the hadronic calorimeter. The absorber in the CCFH is a uranium-niobium (2\%) alloy while the CCCH uses copper. The segmentation in the hadronic modules is similar to that in the 


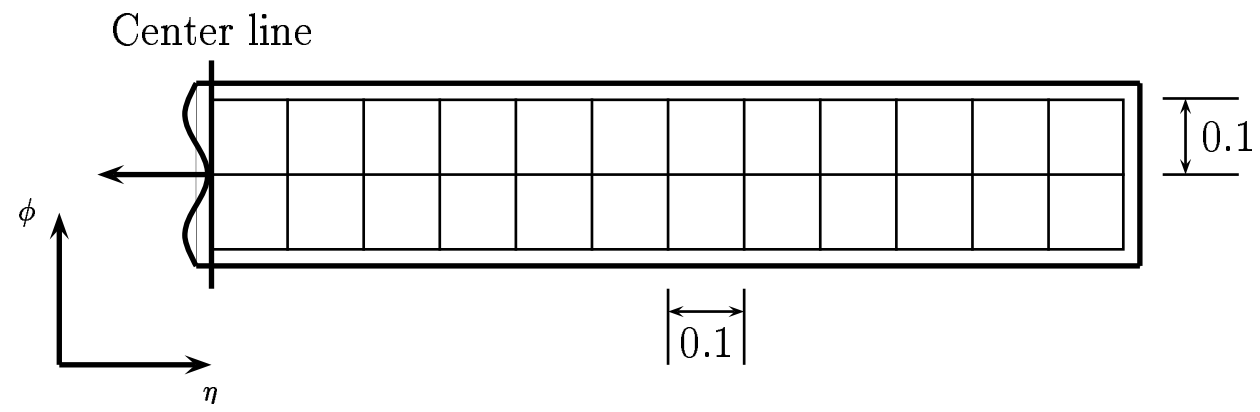

Figure 3.4: The pad design used in the central calorimeter signal boards.

CCEM. Transversely, the signal boards are the same maintaining a $0.1 \times 0.1$ pseudorapidity/azimuth pad structure. Longitudinally, the CCFH's 50 unit cells are read out three times. The first layer contains 21 cells, the second 16 cells, and the third 13 cells. The CCCH is read out only once. The four layers of the CCEM and the first layer of the $\mathrm{CCFH}$ provide over 40 radiation lengths allowing for complete containment of electromagnetic showers.

\subsubsection{Calorimeter Electronics}

The charge collected in each liquid argon gap on the individual copper pads is carried to the edge of the modules by copper traces etched on the interior copper cladding of the signal boards. Signals are then ganged as described earlier by connecting these traces by thin wires which also connect to a readout board. The readout board design is similar to that of the signal board. Copper traces are etched out of the copper cladding on one of two G10 boards which are then laminated together. These traces carry the ganged signals from the interior of the calorimeter to either face. From there, coaxial 


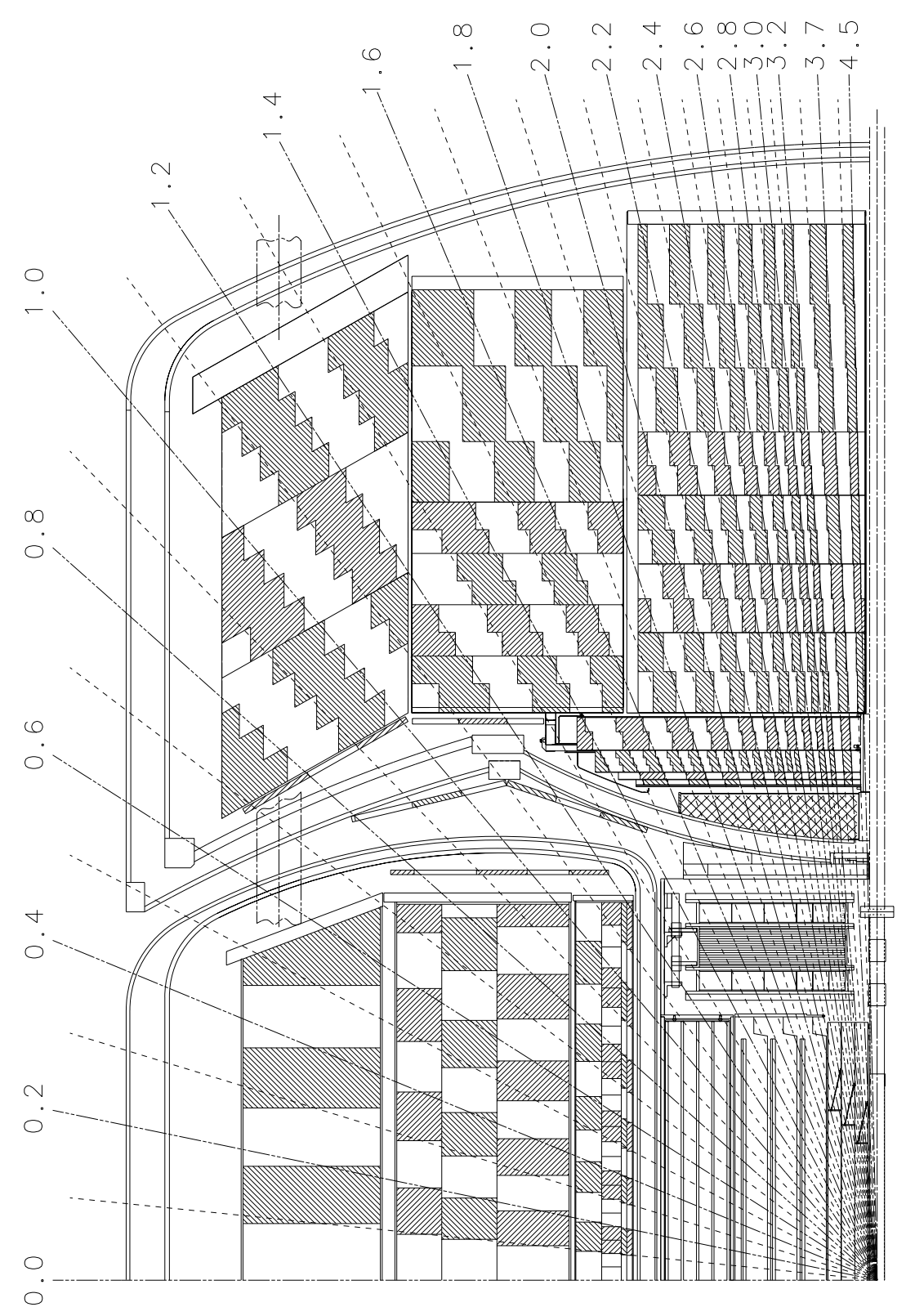

Figure 3.5: A quarter view of the $\mathrm{D} \emptyset$ calorimeter showing the coverage in $\eta$. 
cables carry the signals to a feedthrough board, transferring them from inside the cryostat to the outside. Twisted pair cables connect the warm side of the feedthroughs to charge sensitive preamplifiers. These are located in close proximity of the detector to reduce noise. The output of the preamplifiers is transferred via $\sim 30 \mathrm{~m}$ twisted pair cables to a baseline subtractor (BLS) shaping and sampling circuit. The BLS samples the signal just prior to the beam crossing (base) and again $2.2 \mu \mathrm{s}$ (peak) later. The difference between the base and peak is obtained as a "dc" voltage which is then digitized. Depending on the signal size, the BLS output is amplified by a factor of 1 or 8 to increase the dynamic range of the electronics. The digitization is performed by 24 channel 12 bit ADC's. Together with the BLS amplification, this provides a total dynamic range of $2^{15}$. The gain parameters have been set so that 1 ADC count corresponds to approximately $3.75 \mathrm{MeV}$.

Calibration is maintained by injecting a fixed charge from a pulser through a precision resistor to the input of the preamplifier. A system has been designed to deliver a uniform pulse to all channels. The system has been shown to be accurate and stable to better than $.25 \%[20]$.

\subsubsection{Trigger and Data Acquisition}

The data acquisition system for the $\mathrm{D} \emptyset$ detector can write events to tape at a rate of a few hertz. This is a problem since the Tevatron with six bunches each of protons and antiprotons produces a collision rate on the order of 0.5 $\mathrm{MHz}$. A triggering system is necessary to filter the events and reduce the raw 
collision rate to one that can be saved for later analysis. Such a system of hardware and software tools in three layers has been built and is operating at $\mathrm{D} \emptyset$.

The primary purpose of the first layer (level 0 ) is to identify inelastic collisions. The trigger consists of an array of scintillation counters located on the front face of the end calorimeters. Requiring both scintillators to fire in coincidence rejects beam-gas events (protons or antiprotons interacting with the residual gas in the beampipe) and is the first evidence that something interesting has occurred. In addition, an initial $\mathrm{z}$ position of the primary vertex is determined using relative timing information. This vertex is used in subsequent levels of the trigger system.

The second layer (level 1) performs some coarse energy flow calculations based on information from the calorimeter. Analog signals are taken from the BLS and summed into trigger towers whose size is $\Delta \eta=\Delta \phi=0.2$ for $|\eta| \leq$ 4.0. Separate sums for the electromagnetic and hadronic components as well as their totals are computed. The transverse energy flows are also computed by weighting the trigger tower energies by $\sin \theta$ where the polar angle is computed assuming the nominal vertex $(\mathrm{z}=0)$. The trigger tower energies are then added to produce global sums of electromagnetic energy, hadronic energy in addition to their transverse components and the total energy. The $\mathrm{x}$ and $\mathrm{y}$ components of the transverse energy are used to calculate the missing transverse energy of the event $\left(H_{T}\right)$. All of these quantities can be used to generate a level 1 trigger.

An event that generates such a level 1 trigger is then passed to the last 
layer of the trigger (level 2). This trigger consists of 50 nodes (VAX 400060 microprocessors) connected by high speed data cables to the digitization electronics of the detector subsystems. The nodes collect the data from the data cables in a coherent fashion, building the event structure. Based on the level 1 trigger, a combination of software tools are run on the event. These tools look for a particular particle or event characteristic. Events producing positive results in these tools are then written to disk and eventually spooled to tape for further analysis[20]. 


\section{Chapter 4}

\section{The 1991 Testbeam}

\subsection{Testbeam goals}

During the second half of $1991, \mathrm{D} \emptyset$ conducted a testbeam in the neutrino west $(\mathrm{NW})$ beamline in the fixed target area at Fermilab. This effort concentrated on the electromagnetic and hadronic modules of the central calorimeter. The goals of this testbeam included the calibration of the detector and the verification of the Monte Carlo simulation. To correctly extract the physics from the collider data, it is necessary to determine the detector energy resolution and to understand its uniformity of response. The simulation is necessary to understand the details of the interactions that take place in the detector providing information to properly reconstruct the events. Together, the testbeam results and the simulation are needed to properly derive a value of the mass of the $\mathrm{W}^{ \pm}$from the collider data. 


\subsection{Testbeam Configuration}

In order to obtain a calibration that was accurate and applicable in the collider configuration, a complicated system was developed. It consisted of an instrumented beamline that was capable of delivering a beam of particles of known momentum to a module array designed to model the details of the full central calorimeter.

The beamline configuration is shown in Fig. 4.1. The critical elements include the momentum analyzing magnet NW9E. In conjunction with the proportional wire chamber (PWC) tracking system, an absolute value for the momentum of individual tracks is determined to an accuracy of $\sim 0.5 \%[21]$. Scintillating tiles are used to both trigger on particles as well as veto particles outside of a well defined beam spot. Cerenkov light detectors placed in the beamline provided particle identification.

A cryostat containing the liquid argon was located at the end of the beamline. Inside were placed eight CC modules: four electromagnetic (EM), two fine hadronic $(\mathrm{FH})$, and two coarse hadronic $(\mathrm{CH})$ (Fig. 4.2). The modules were arranged as they would be if they were located in the full calorimeter. The cryostat featured thin windows for passage of the beam to reduce upstream losses. In addition, it had the ability to rotate allowing the beam to be directed at any calorimeter tower at various angles of incidence (Fig. 4.3). The liquid argon in front of the module array was displaced by a low density material (Rhoacell ${ }^{\mathrm{TM}}$ ) to reduce the amount of upstream material. To mimic the two $1 / 4$ " cryostat walls of the central calorimeter, a $1 / 2 "$ piece of stainless steel 


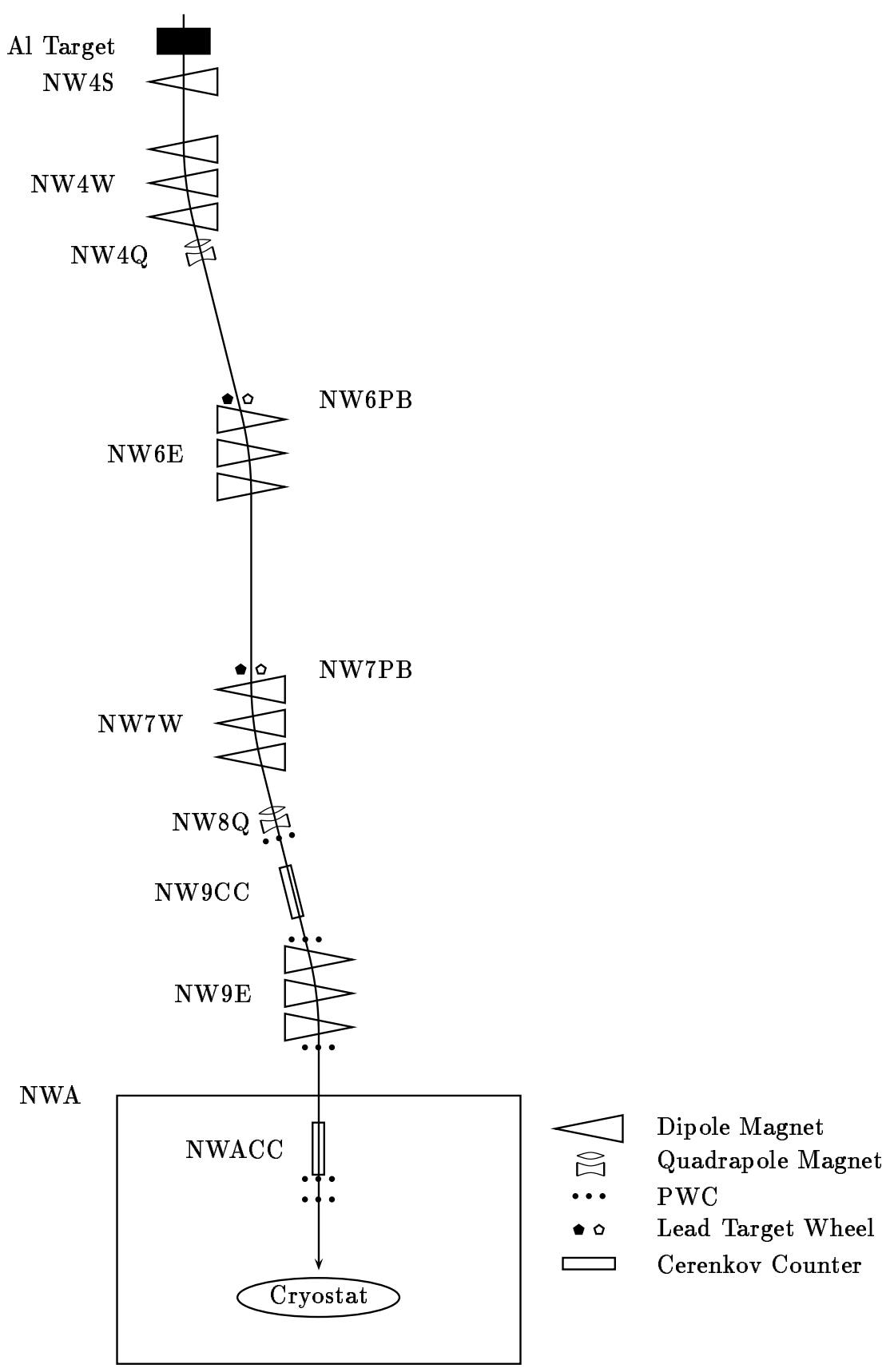

Figure 4.1: Beamline used in the 1991 testbeam. 


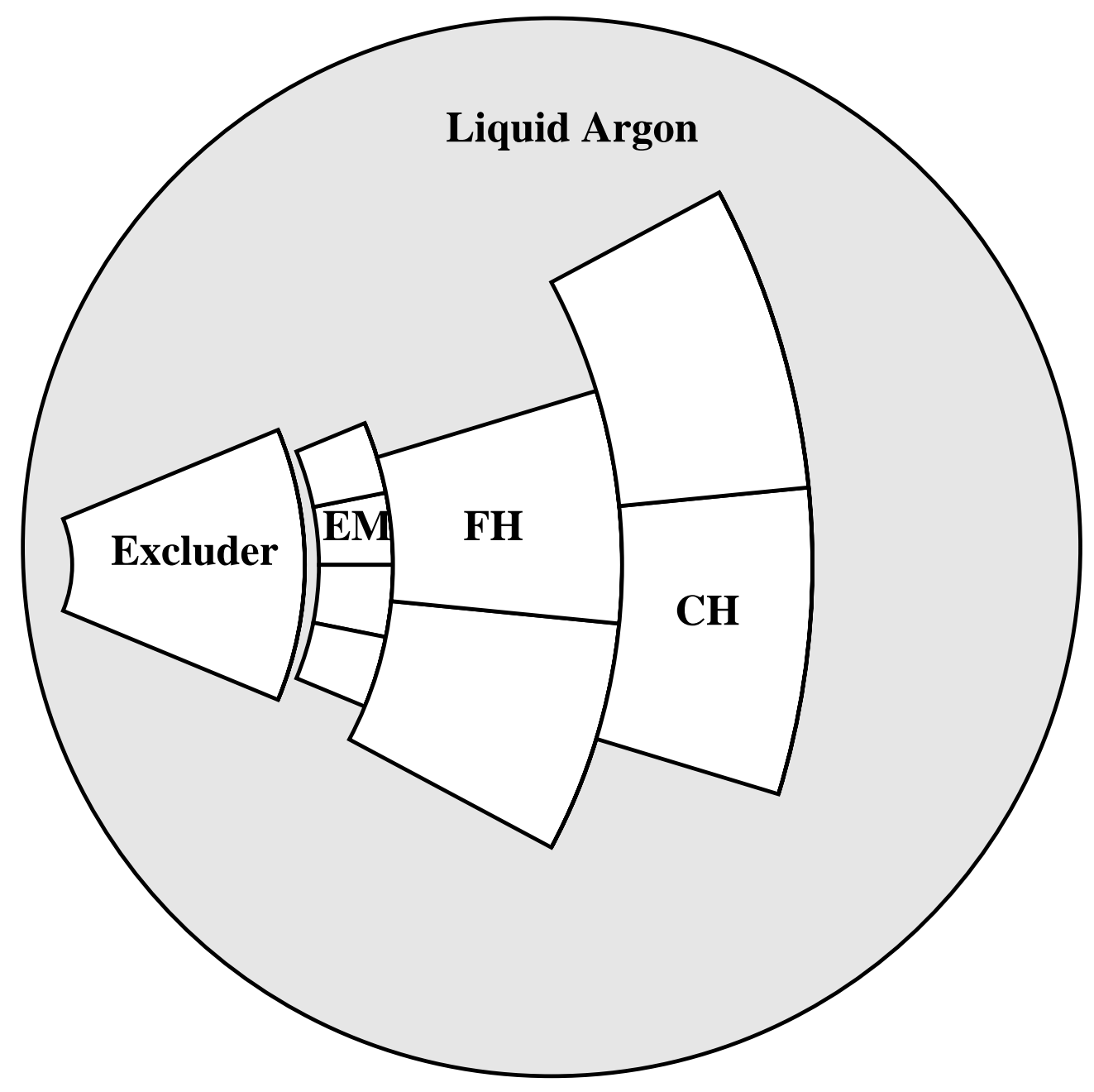

Figure 4.2: Configuration of the 1991 testbeam. 


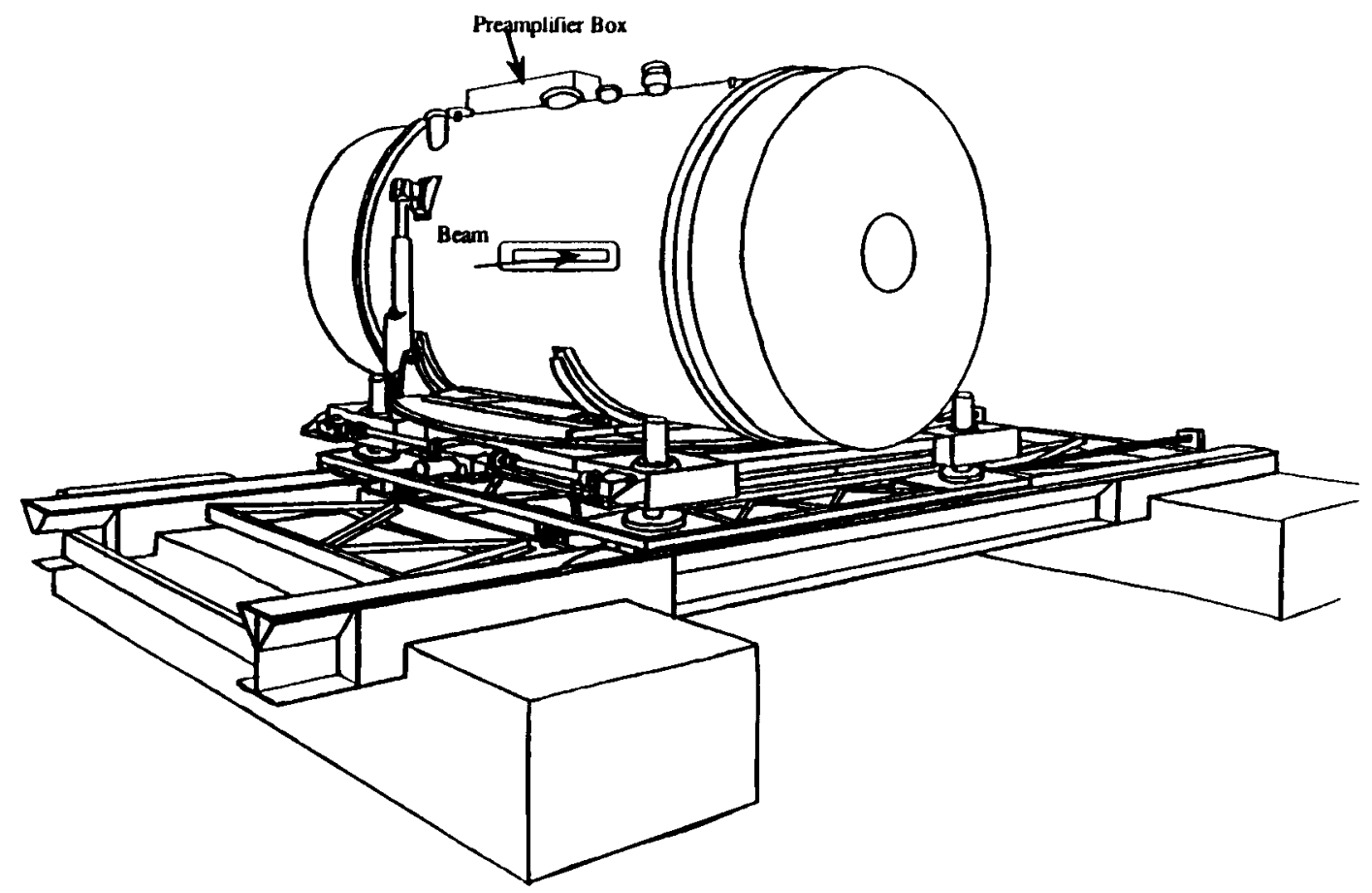

Figure 4.3: The cryostat and transporter system used in the 1991 testbeam. 
was placed in front of the module array.

The electronics used were similar to that used at the collider. The signals were brought out of the cryostat to the preamplifiers in the same way that they are at $D \emptyset$. Although the cables from the preamplifiers to the baseline subtractors were longer than those used in the collider, corrections have been applied to account for the signal attenuation. ADC's then digitized the output of the baseline subtractors.

\subsection{Testbeam Simulation}

An extremely detailed simulation of the testbeam configuration was written using the CERN packages GEANT[22] to aid in collider data analysis. This simulation modeled the calorimeter modules at the plate level. A description of the upstream material inside the cryostat was included as well as the beamline elements downstream of the momentum analyzing magnet NW9E.

Since upstream interactions and energy losses lead to nongaussian tails in the energy distributions and removal of these events is difficult, potentially resulting in a biased data sample, the upstream material in the beamline and the cryostat was included in the simulation. The beamline elements that were modeled and their locations relative to the cryostat are shown in Tab. 4.1 and Tab. 4.2. This includes four PWC's, two scintillator tiles, and two cerenkov counters. The PWC's were modeled as a two layer device, one consisting of the gas volume and the other a combination of all nongaseous elements[23]. The 


\begin{tabular}{||lccc||}
\hline Detector & Material & $\begin{array}{c}\text { Thickness } \\
(\mathrm{cm})\end{array}$ & $\begin{array}{c}\text { Radiation Lengths } \\
\left(X_{0}\right)\end{array}$ \\
\hline PWC & & & \\
gas & $\mathrm{Ar} / \mathrm{CO}_{2}(80 \% / 20 \%)$ & 1.91 & 0.0002 \\
window & aluminum & 0.0064 & 0.0007 \\
\hline ASC & scintillator & 0.64 & 0.0154 \\
\hline CKVA & & & \\
gas & 3.5psi He & 2450.0 & 0.0012 \\
windows $(2)$ & titanium & $0.02 / 0.03$ & 0.0143 \\
mirror & lucite & 0.15 & 0.0044 \\
\hline CKV2 & & & \\
gas & nitrogen & 203.2 & 0.0181 \\
windows $(2)$ & titanium & 0.02 & 0.0114 \\
mirror & lucite & 0.20 & 0.0059 \\
\hline
\end{tabular}

Table 4.1: 1991 Testbeam Load 2 Beamline Parameters. Included are proportional wire chambers (PWC), scintillators (ASC), and two Cerenkov counters (CKVA, CKV2).

\begin{tabular}{||lc||}
\hline $\begin{array}{l}\text { Beamline } \\
\text { element }\end{array}$ & $\begin{array}{c}\text { Distance } \\
\text { upstream }(\mathrm{cm})\end{array}$ \\
\hline PWC9-2 & 3743.96 \\
CKVA & 2466.34 \\
ASC1 & 1192.53 \\
PWCA-1 & 1186.18 \\
PWCA-2 & 406.40 \\
ASC3 & 401.32 \\
CKV2 & 203.20 \\
PWCA-C & 5.08 \\
\hline
\end{tabular}

Table 4.2: Location of the beamline elements relative to the front of the testbeam cryostat. Included are proportional wire chambers (PWC), scintillator tiles (ASC), and Cerenkov detectors (CKV). 


\begin{tabular}{||cccc||}
\hline Element & Material & $\begin{array}{c}\text { Thickness } \\
(\mathrm{cm})\end{array}$ & $\begin{array}{c}\text { Radiation Lengths } \\
\left(X_{0}\right)\end{array}$ \\
\hline Outer Cryo Window & steel (Fe) & 0.16 & 0.09 \\
Inner Cryo Window & steel (Fe) & 0.16 & 0.09 \\
Liquid Argon Excluder & Rohacell & 58.6 & 0.10 \\
CC Cryostat Wall & & & \\
Simulator & steel (Fe) & 1.27 & 0.72 \\
Liquid Argon & liquid argon & 2.50 & 0.18 \\
\hline
\end{tabular}

Table 4.3: Elements upstream of the calorimeter modules inside the testbeam cryostat.

Cerenkov counters consisted of a cylindrical gaseous volume with a disc shaped titanium window located on both ends and lucite mirror on the downstream end[24]. Inside the cryostat, the beamline cryostat windows, the liquid argon excluder, and the CC cryostat wall simulator were modeled. Tables 4.1 and 4.3 show the amount of material in front of the calorimeter modules in radiation lengths.

In the modules, the details at the unit cell level were reproduced (sec. 3.2). This includes the absorber plates, signal and readout boards, copper pads, and liquid argon gaps. The relevant dimensions for the CCEM modules are given in Tab. 4.4 and for the CCFH module in Tab. 4.5. Although the length and thickness of module elements do not change as a function of location in the module, due to the wedge shape the widths do change. The CCEM simulation contains over 140 individually placed volumes while the CCFH contains over 260 . 


\begin{tabular}{||lccc||}
\hline Element & Thickness & Length & Width \\
\hline absorber (U) & $3 \mathrm{~mm}$ & $260.5 \mathrm{~cm}$ & $16.1-19.5 \mathrm{~cm}$ \\
signal board $(\mathrm{g}-10)$ & $1 \mathrm{~mm}$ & $260.5 \mathrm{~cm}$ & $16.0-19.7 \mathrm{~cm}$ \\
readout board $(\mathrm{g}-10)$ & $1.6 \mathrm{~mm}$ & $260.5 \mathrm{~cm}$ & $16.0-19.7 \mathrm{~cm}$ \\
copper pads $(\mathrm{Cu})$ & $0.08 \mathrm{~mm}$ & $260.3 \mathrm{~cm}$ & $15.8-19.5 \mathrm{~cm}$ \\
liquid argon gaps & $2 \times 2.3 \mathrm{~mm}$ & $260.3 \mathrm{~cm}$ & $15.8-19.5 \mathrm{~cm}$ \\
frontplate (Fe) & $3.2 \mathrm{~mm}$ & $261.1 \mathrm{~cm}$ & $16.5 \mathrm{~cm}$ \\
backplate $(\mathrm{Fe})$ & $3.2 \mathrm{~mm}$ & $261.1 \mathrm{~cm}$ & $19.8 \mathrm{~cm}$ \\
sideskins $(\mathrm{Fe})$ & $1.9 \mathrm{~mm}$ & $261.1 \mathrm{~cm}$ & $19.9 \mathrm{~cm}$ \\
endplate $(\mathrm{Fe})$ & $1.8 \mathrm{~cm}$ & $20.9 \mathrm{~cm}$ & $16.5-20.6 \mathrm{~cm}$ \\
\hline
\end{tabular}

Table 4.4: Dimensions of the elements modeled in the CCEM module.

\begin{tabular}{||lccc||}
\hline Element & Thickness & Length & Width \\
\hline absorber $(\mathrm{U})$ & $6 \mathrm{~mm}$ & $259.3 \mathrm{~cm}$ & $41.2-64.2 \mathrm{~cm}$ \\
signal board $(\mathrm{g}-10)$ & $1 \mathrm{~mm}$ & $259.3 \mathrm{~cm}$ & $41.5-64.6 \mathrm{~cm}$ \\
readout board $(\mathrm{g}-10)$ & $1.6 \mathrm{~mm}$ & $259.3 \mathrm{~cm}$ & \\
copper pads $(\mathrm{Cu})$ & $0.08 \mathrm{~mm}$ & $259.1 \mathrm{~cm}$ & $41.3-64.4 \mathrm{~cm}$ \\
liquid argon gaps & $2 \times 2.3 \mathrm{~mm}$ & $259.1 \mathrm{~cm}$ & $41.3-64.4 \mathrm{~cm}$ \\
frontplate $(\mathrm{Fe})$ & $4.7 \mathrm{~mm}$ & $259.7 \mathrm{~cm}$ & $41.9 \mathrm{~cm}$ \\
backplate $(\mathrm{Fe})$ & $4.7 \mathrm{~mm}$ & $259.7 \mathrm{~cm}$ & $64.9 \mathrm{~cm}$ \\
sideskins $(\mathrm{Fe})$ & $2.7 \mathrm{~mm}$ & $259.7 \mathrm{~cm}$ & $61.1 \mathrm{~cm}$ \\
endplate $(\mathrm{Fe})$ & $3.2 \mathrm{~cm}$ & $64.5 \mathrm{~cm}$ & $41.2-66.4 \mathrm{~cm}$ \\
\hline
\end{tabular}

Table 4.5: Dimensions of the elements modeled in the CCFH module. 


\section{Chapter 5}

\section{Energy Scans}

One of the goals of the 1991 testbeam effort was to determine the energy measurement resolution of the central electromagnetic calorimeter. In addition, we desired to confirm the ability of the Monte Carlo simulation to reproduce this aspect of the detector's performance. Both of these are important in extracting and understanding the $\mathrm{W}^{ \pm}$mass from collider data. To complete these tasks, three energy scans were conducted, at pseudorapidities of $0.05,0.45$, and 1.05 using monochromatic beams of electrons with energies ranging from 10 to $150 \mathrm{GeV}$. These scans were then duplicated with the simulation. This chapter will explain the event selection process and present the results of the comparison between the testbeam data and the simulation.

\subsection{Data Set}

The three energy scans were conducted at $\eta$ 's of $0.05,0.45$, and 1.05 . The scans included $10,15,20,25,30,40,50,75,100,125$, and $150 \mathrm{GeV}$ 
electron runs. Approximately 10,000 events were taken at each energy at each pseudorapidity. All runs were analyzed using the analysis packages TB90L2_SURVEY[25] and TB90L2_GAINCOR[26]. These packages provide millimeter accuracy for tracking and the best gain corrections for the testbeam setup. All the corrections incorporated in the CAHITS package were also used in the analysis. CAHITS corrections included pedestal subtraction and initial gain corrections, the sampling fraction correction, and a conversion from ADC counts to energy in $\mathrm{GeV}$. All energy sums used a $5 \times 5$ window $(\Delta \eta \times \Delta \phi=0.5 \times 0.5)$ extending over all four layers of the electromagnetic and the first section of the fine hadronic module.

In order to ensure the quality of the data and to enable a momentum correction, cuts were made using the beam tracking system. One and only one reconstructed track was required in the vertical tracking plane as well as one and only one reconstructed momentum (measured in the horizontal plane). These cuts varied in efficiency from $15 \%$ to $60 \%$. Tables $5.1,5.2$, and 5.3 show the number of events surviving after each cut for each run.

After this initial selection, cuts were made to remove electrons near the intermodule $\phi$ crack, electrons that had hit apparatus upstream of the calorimeter, pions, and muons. All such events cause low energy tails in the resulting energy distribution. The typical cut to remove electrons in the $\phi$ crack was to require the projected track to be at least $0.2 \phi$ units from the crack (see figure 5.1). These events can be easily identified by an increase in the energy deposited in the first electromagnetic layer. Cuts were made in both $\eta$ and $\phi$ (see figures 5.2 and 5.3). Since these cuts were a function of the 


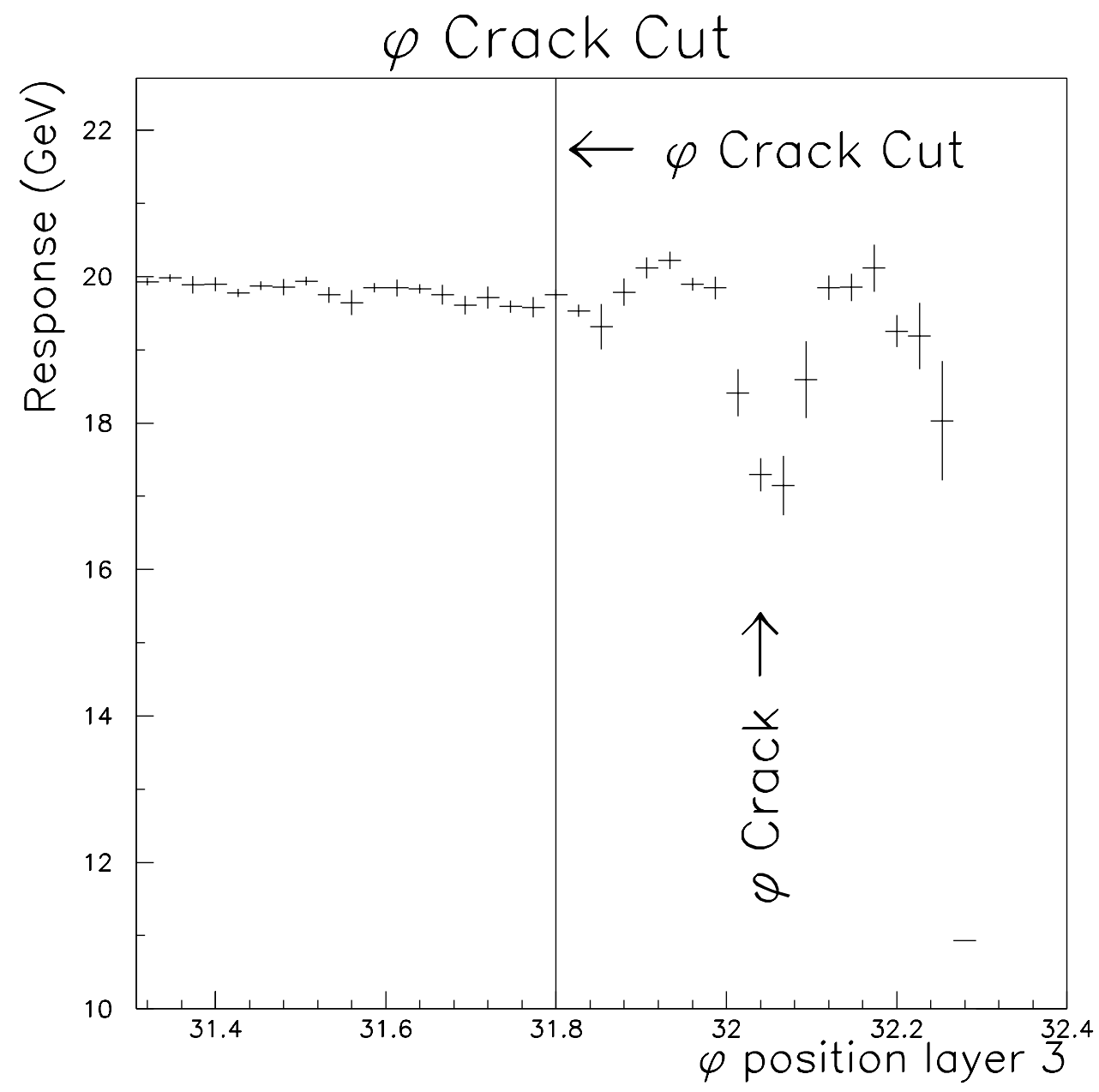

Figure 5.1: Response vs. the extrapolation of the reconstructed vertical track into the third electromagnetic layer for run $13842(20 \mathrm{GeV})$. The crack itself is located at $\phi=32.06$. The cut was made at 31.8 to remove events that are affected by the crack. 
beamline and not the modules themselves, the cuts varied from run to run. Although pions could have been removed using the Cerenkov counters, these proved to be rather inefficient. Instead, a cut requiring less than $10 \%$ of the beam energy be present in the first layer of the fine hadronic module was used. Very few events were removed in this fashion. Muons were removed using a counter behind the detector array. This too removed very few events.

Finally, a ten standard deviation energy cut was made about the mean of a fitted gaussian to remove residual pedestals and other low energy anomalies (Tab. 5.1, 5.2, 5.3).

Using these final data sets, gaussians were fitted for each energy and $\eta$. The $\chi^{2}$ for each fit is recorded in Tab. 5.7, 5.8, and 5.9. The mean and sigma was used to calculate a resolution for each fit.

\subsection{Monte Carlo Data Set}

Due to the limited computer resources, not all of the testbeam data runs were simulated with the Monte Carlo. At each pseudorapidity, electron runs of $10,25,50,75,100,125$, and $150 \mathrm{GeV}$ were produced, each with 500 events. The beam transport included all of the upstream instrumentation (Sec. 4.3). All particles were tracked in the simulation down to an energy of $10 \mathrm{KeV}$. When a particle's energy dropped below this cutoff, the particle was stopped

and its energy deposited locally. The Monte Carlo track was defined by the particle's position and direction at the time it entered the testbeam cryostat. This track was used to define the position of the shower. 


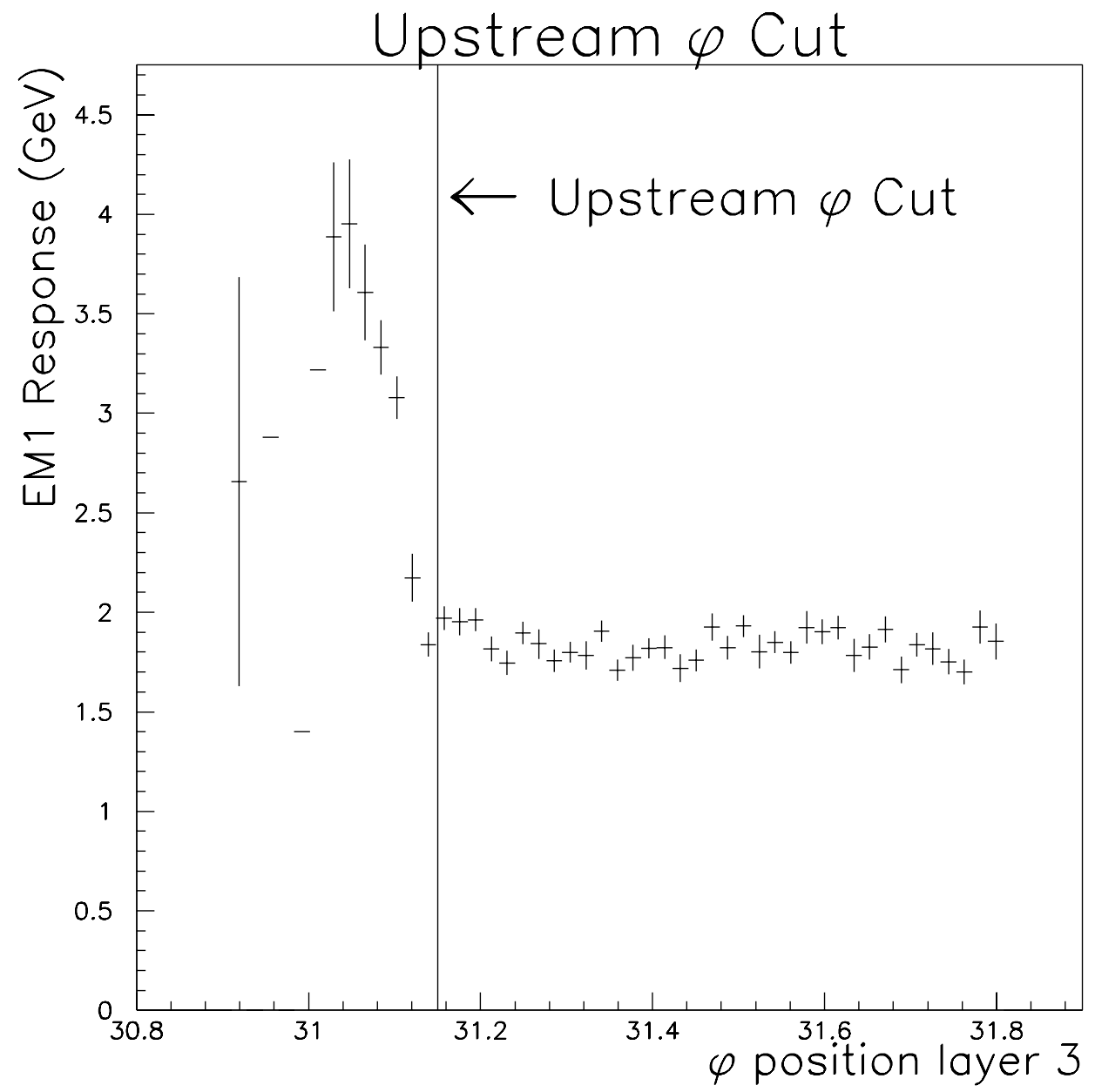

Figure 5.2: Response in the first electromagnetic section vs. the extrapolation of the reconstructed vertical track into the third section for run 13842 (20 $\mathrm{GeV}$ ). We required $\phi>31.15$ to remove any events that may have hit upstream obstructions. 


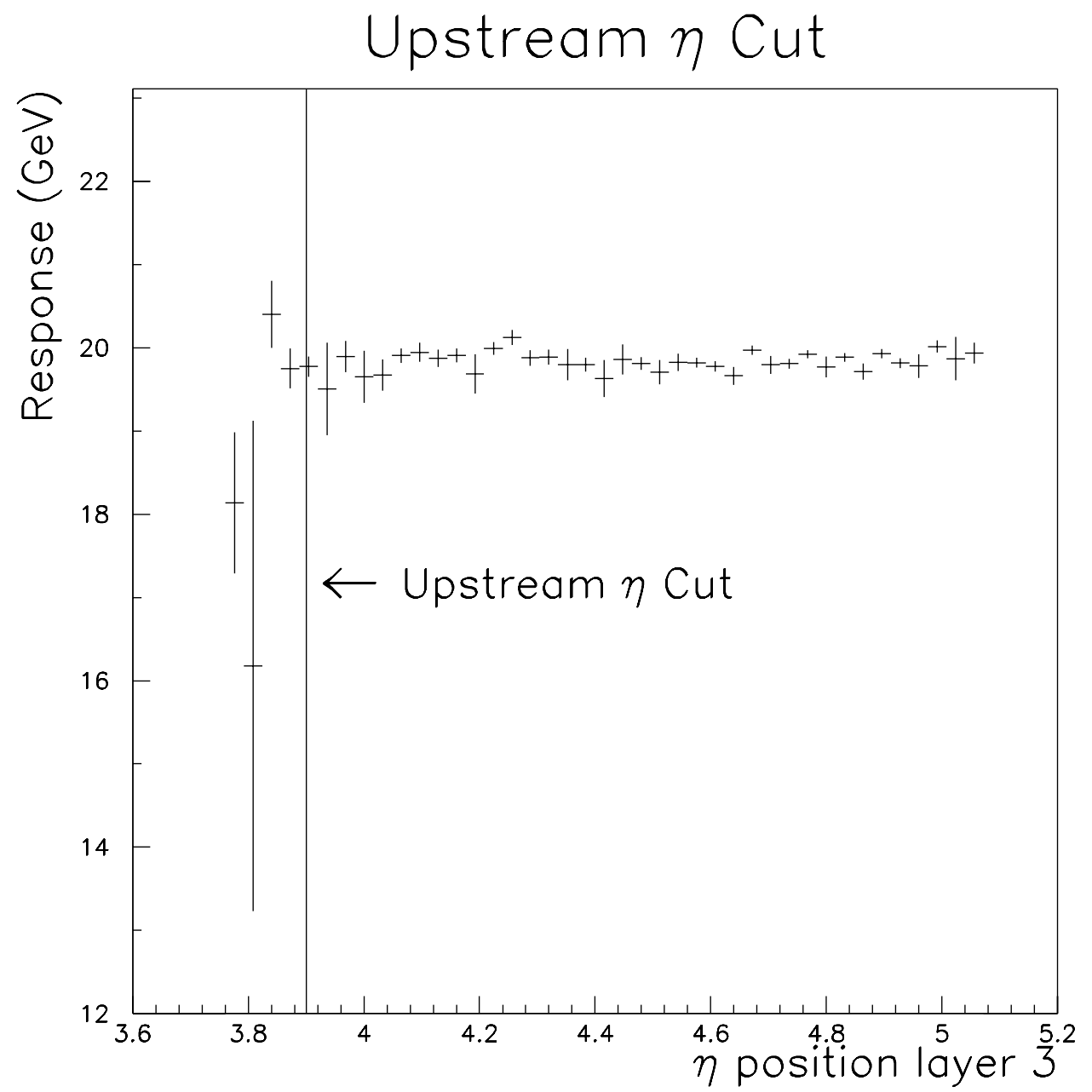

Figure 5.3: Response vs. the extrapolation of the reconstructed horizontal track into the third electromagnetic section for run $13842(20 \mathrm{GeV})$. A cut was made at $\eta>3.9$ to remove any events that may have hit upstream obstructions. 
The same methodology was employed to make cuts on the Monte Carlo data as was used on the real data. The tracking cuts were fully efficient in the simulation. Since none of the beamline support structures were modeled in the simulation, very few events were cut by the upstream requirements. The intermodule crack was included and did result in some loss of events. The pion cut was also made in the simulation. As in the data, less than $10 \%$ of the beam energy was required to be in the first layer of the fine hadronic module. Due to the extremely low number of Monte Carlo events removed, (at most 2 out of 500 ), we believe this cut to be fully efficient for electrons in the real dataset. The muon counter was not implemented in the simulation so no cut was made. A 10 standard deviation energy cut was not necessary for the Monte Carlo data.

Most of the events that were rejected at low energy but not lost in the intermodule crack were lost due to the particle scattering out of the beam and hence out of the energy sum window. At higher energies, most events lost were lost due to fluctuations in the amount of energy deposited in the first fine hadronic layer. For $\eta$ of 0.05 and 0.45 , the intermodule crack cut was made at $\phi<31.75$ (the crack was located at $\phi$ of 32.0 ). For $\eta$ of 1.05 , the cut was moved to 31.5 possibly due to the fact that the cut was being made at a deeper section of the shower than at the other $\eta$ 's. The results of the Monte Carlo analysis are presented in Tab. $5.4-5.6$ and $5.10-5.12$. 


\subsection{Energy Resolution}

In the determination of the energy resolution, a resolution function was fitted to the $\operatorname{data}[20]$ :

$$
\left(\frac{\sigma}{\mu}\right)=\sqrt{C^{2}+\frac{S^{2}}{E}+\frac{N^{2}}{E^{2}}}
$$

where $\mathrm{C}$ results from energy dependent contributions to the resolution such as calibration errors, $S$ results from sampling fluctuation contributions to energy resolution, and $\mathrm{N}$ results from energy independent contributions to resolution such as electronic pedestals and uranium noise. There is a high level of correlation between these parameters, however.

In order to perform a precise comparison to the Monte Carlo, the contribution from noise to the total resolution was subtracted. In order to accomplish this, events where there was no beam particle in the detector were taken to measure the zero energy response. These pedestal events were taken during each run. The width of the pedestal energy distribution was taken as a measurement of the amount of noise for each run. This value, $\mathrm{N}$, was then subtracted in quadrature from the fractional energy resolution at each point (Tab. 5.7, 5.8, 5.9). We have:

$$
\begin{aligned}
\left(\frac{\sigma}{\mu}\right)_{\text {noise sub }} & =\sqrt{\left(\frac{\sigma}{\mu}\right)^{2}-\frac{N^{2}}{E^{2}}} \\
& =\sqrt{C^{2}+\frac{S^{2}}{E}}
\end{aligned}
$$




\subsection{Results}

The results of the energy resolution fits are presented in Tab. 5.13. This table also includes the fits for the noise subtracted data. The fits themselves are plotted in figures $5.4-5.9$.

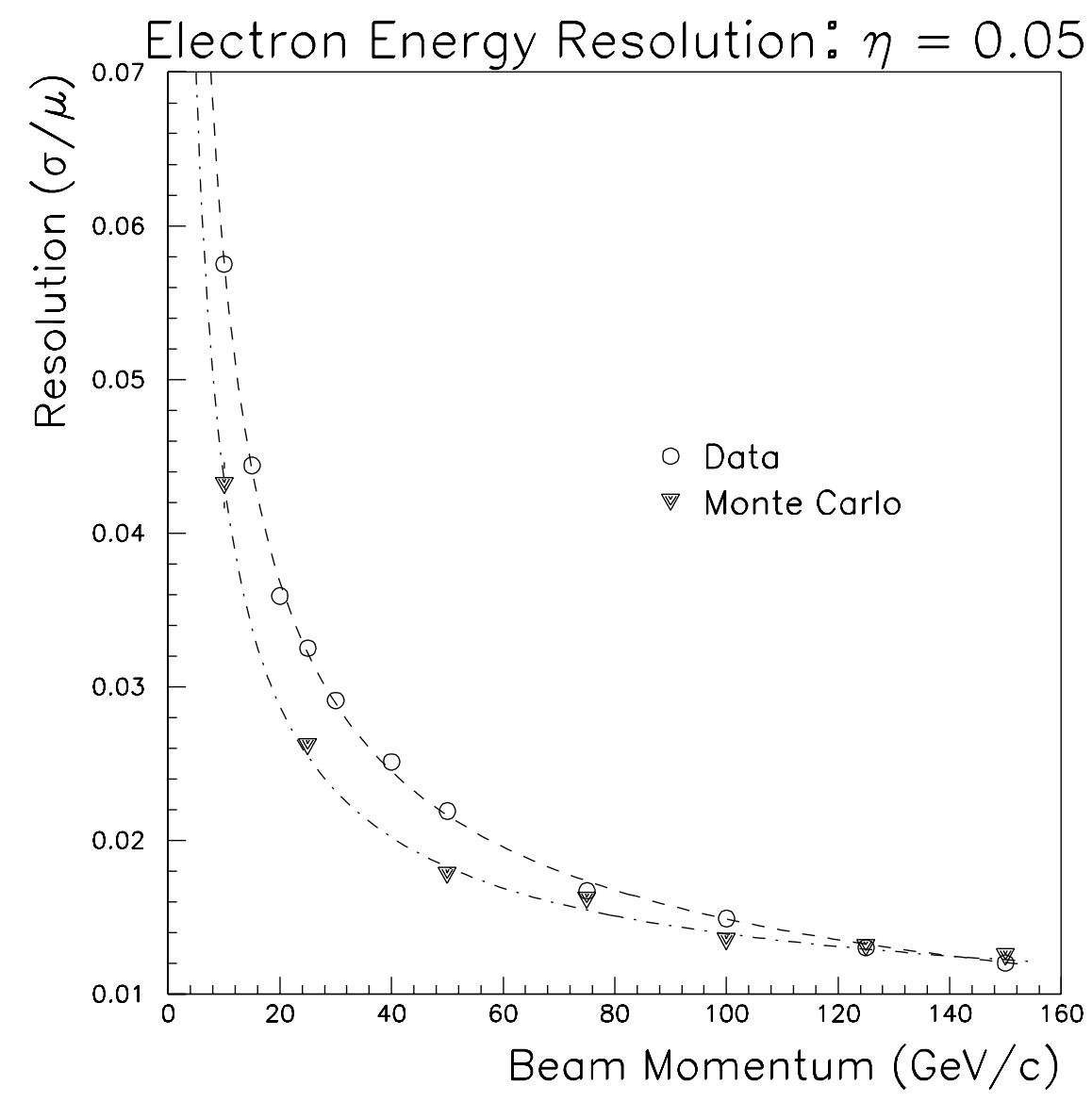

Figure 5.4: Comparison of the simulated and real data resolutions for $\eta=$ 0.05 .

Typically, a small constant term $(<1 \%)$, a sampling term of $\approx 14 \%$, and noise of $350-450 \mathrm{MeV}$ is found. Although this amount of noise as determined 


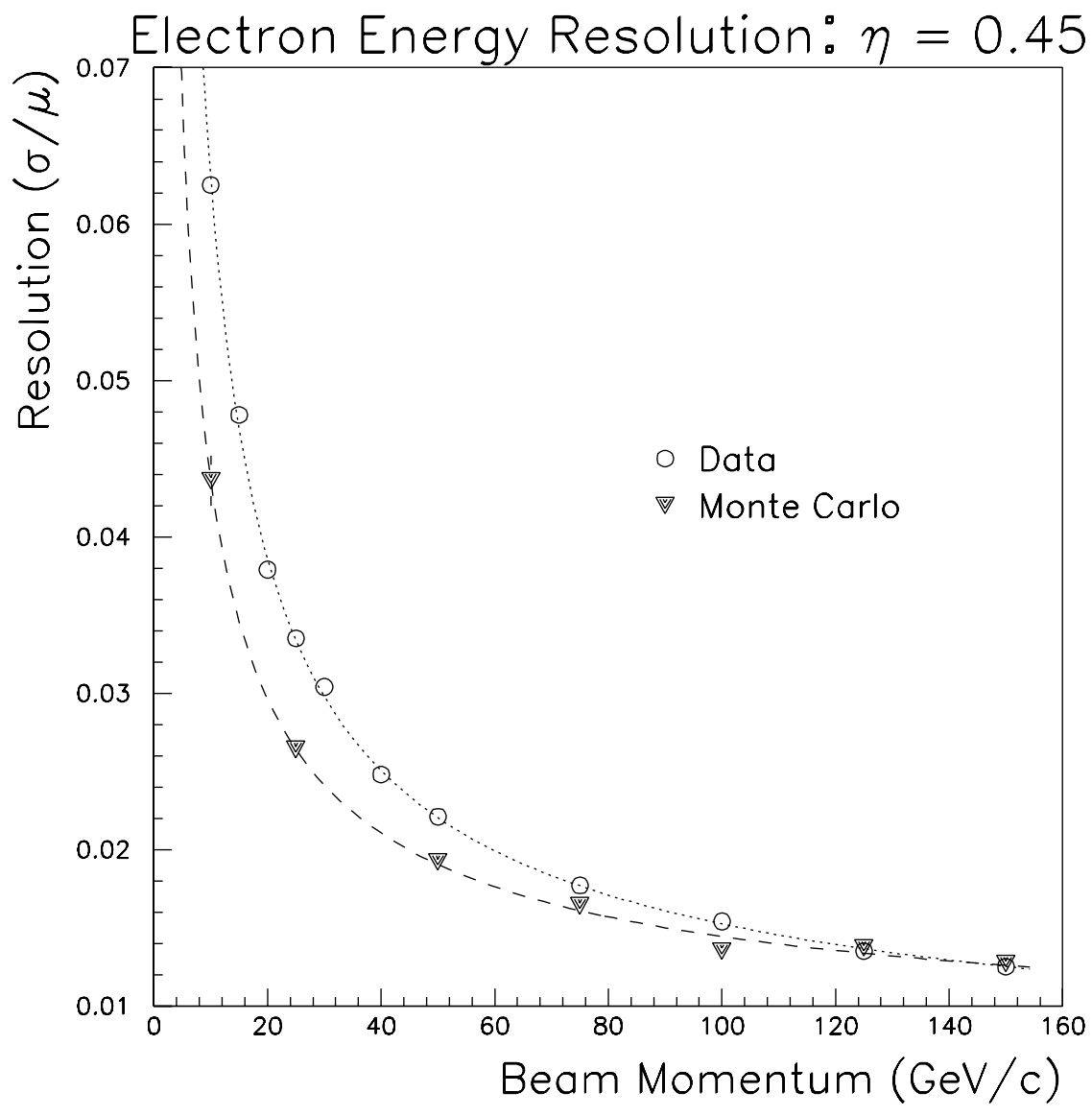

Figure 5.5: Comparison of the simulated and real data resolutions for $\eta=$ 0.45 . 


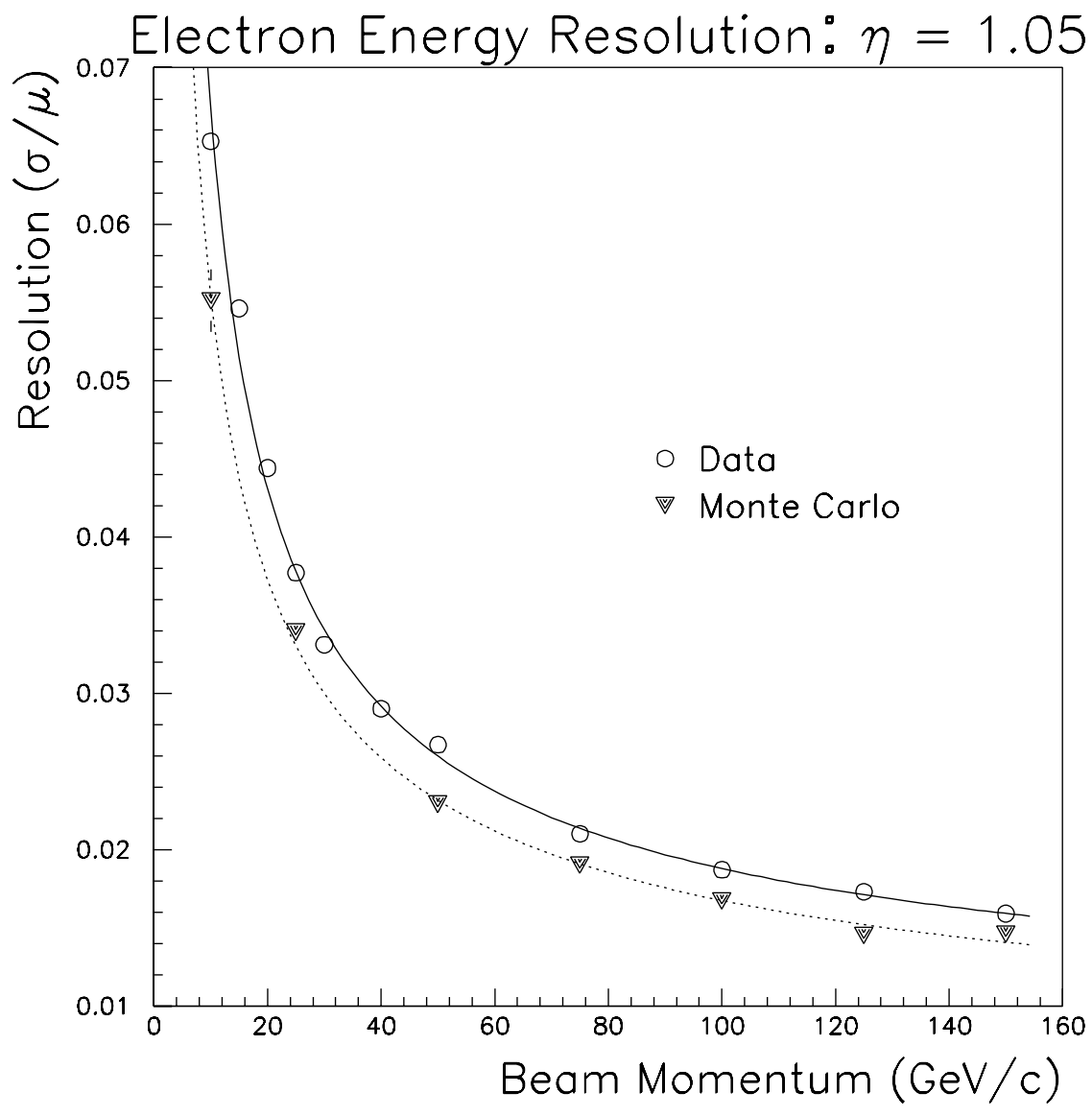

Figure 5.6: Comparison of the simulated and real data resolutions for $\eta=$ 1.05 . 


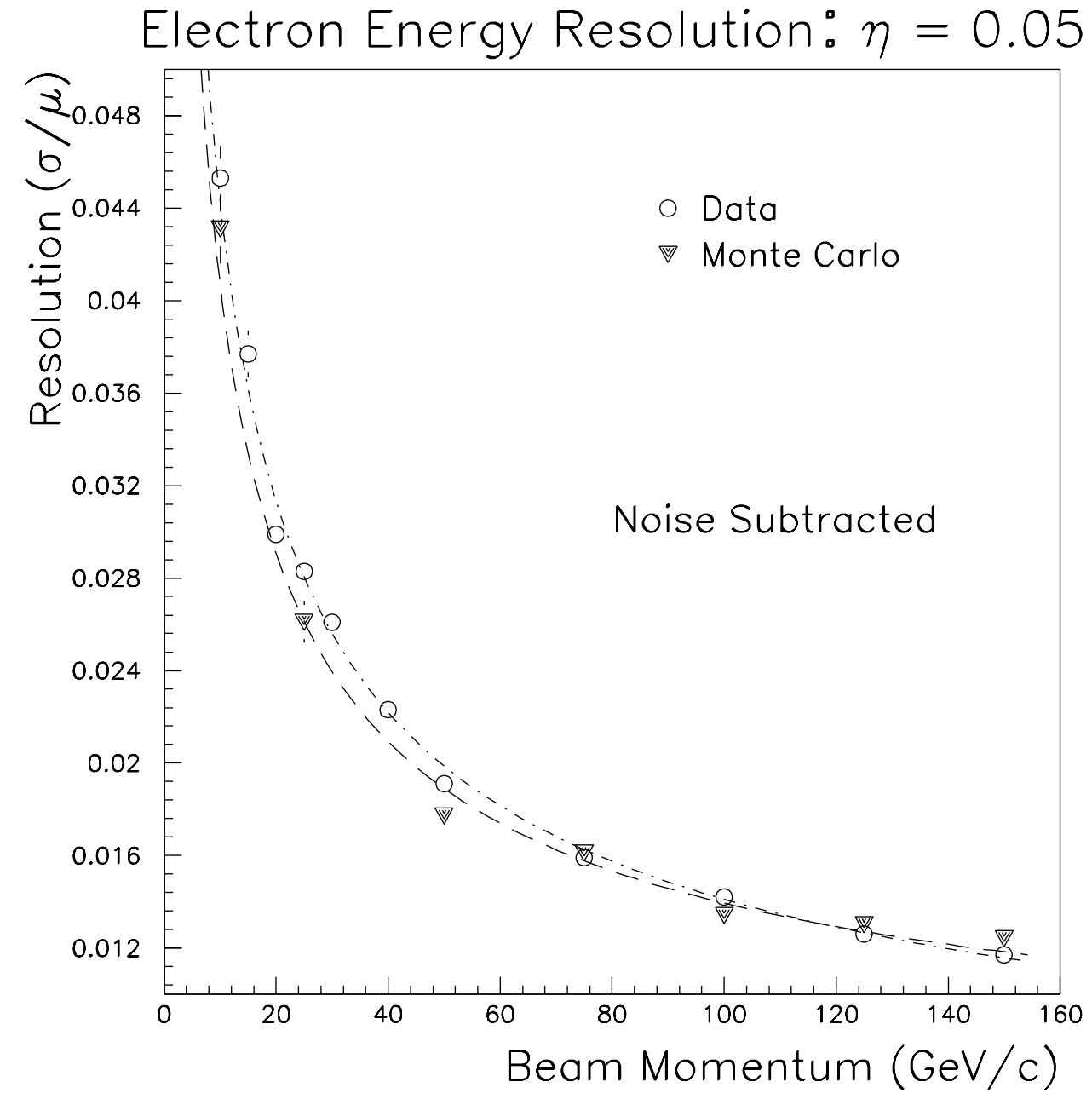

Figure 5.7: Comparison of the simulated and noise subtracted data resolutions for $\eta=0.05$. 


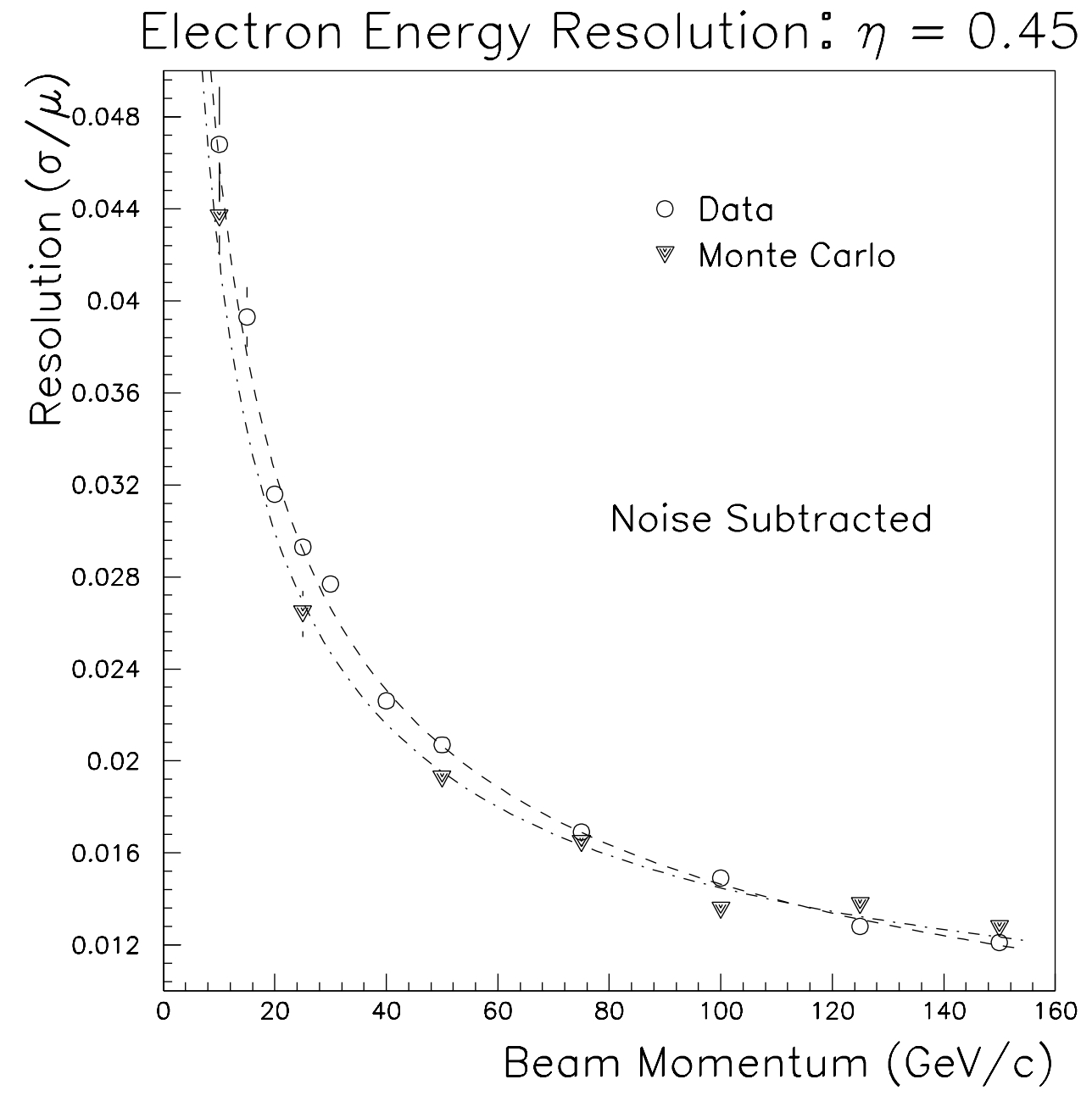

Figure 5.8: Comparison of the simulated and noise subtracted data resolutions for $\eta=0.45$. 


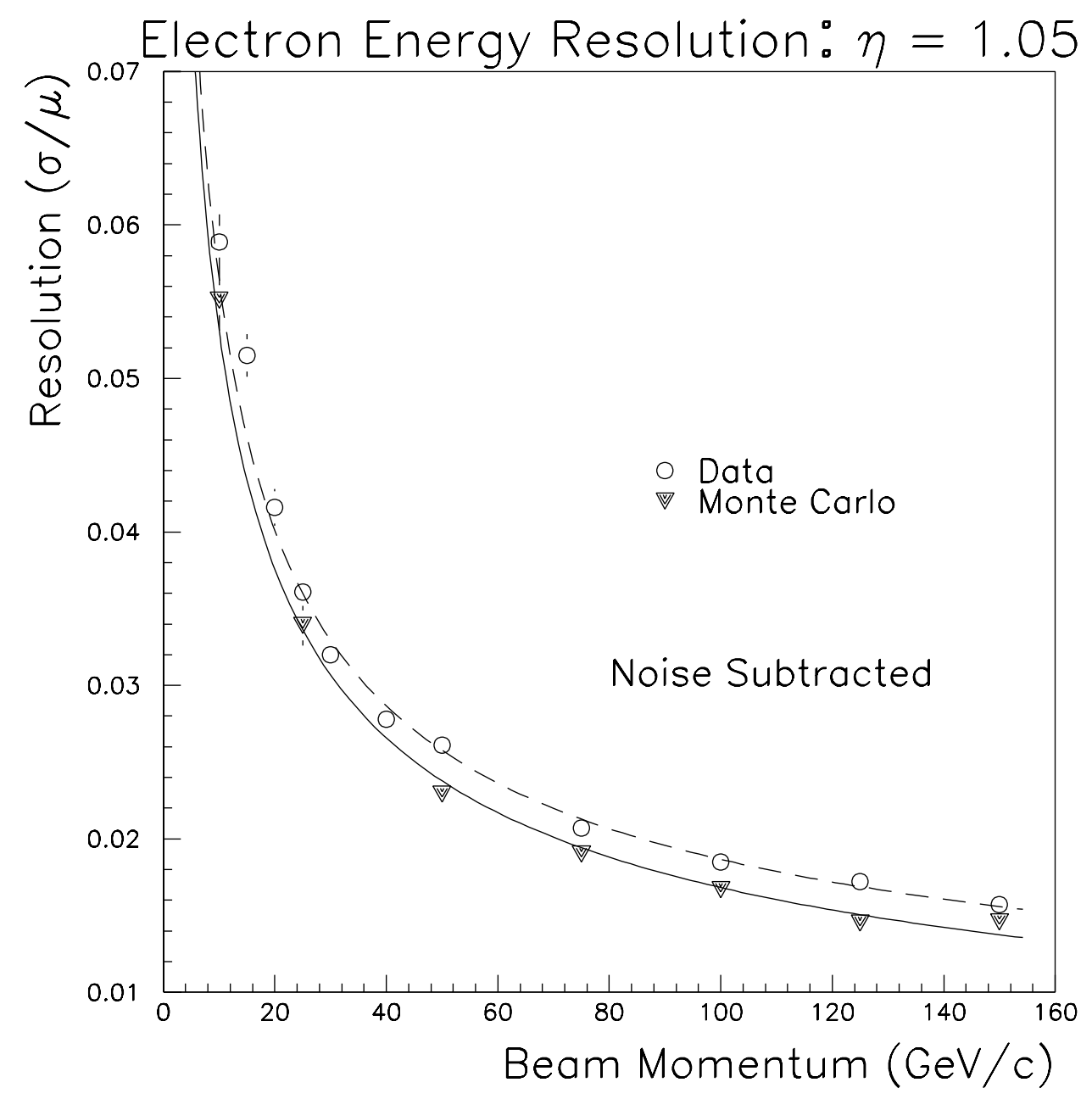

Figure 5.9: Comparison of the simulated and noise subtracted data resolutions for $\eta=1.05$. 
by the fit is consistent with what was determined from the pedestal data, the high degree of correlation between the fit parameters resulted in a nonzero noise term in the fit to the noise subtracted data. For the most part however, forcing the noise to be zero did not result in a worse fit except at $\eta=1.05$.

The comparison between the Monte Carlo and the data for the full resolution is a poor one. At the lower $\eta$ 's (figures 5.4 and 5.5), the agreement improves for the higher energies where noise is less of a factor. At the highest $\eta$ (figure 5.6), this is no longer true.

For the noise subtracted data, the agreement improves for all $\eta$ at low energies where the noise term is important. But there still exists a large discrepancy at high energy at $\eta$ of 1.05. Although the noise subtraction improves the agreement at low energies, significant disagreement exists at higher energy (Figs. $5.7-5.9$ ).

\begin{tabular}{||c|cccccc||}
\hline $\begin{array}{c}\text { Beam } \\
\text { Energy }\end{array}$ & $\begin{array}{c}\text { Tracking } \\
\text { Cut }\end{array}$ & $\begin{array}{c}\phi \\
\text { Crack }\end{array}$ & $\begin{array}{c}\phi \\
\text { Upstream }\end{array}$ & $\begin{array}{c}\eta \\
\text { Upstream }\end{array}$ & $\begin{array}{c}\pi, \mu \\
\text { Cut }\end{array}$ & $\begin{array}{c}10 \sigma \\
\text { Energy Cut }\end{array}$ \\
\hline 10 & 4154 & 3198 & 2865 & 2865 & 2863 & 2848 \\
15 & 3621 & 2941 & 2642 & 2642 & 2497 & 2418 \\
20 & 4329 & 3624 & 3281 & 3281 & 3275 & 3268 \\
25 & 5876 & 5381 & 4798 & 4765 & 4753 & 4735 \\
30 & 5340 & 4956 & 4366 & 4319 & 4263 & 4239 \\
40 & 5953 & 5630 & 5098 & 5097 & 5032 & 5018 \\
50 & 1581 & 1537 & 1376 & 1376 & 1370 & 1370 \\
75 & 2869 & 2740 & 2707 & 2660 & 2637 & 2626 \\
100 & 4443 & 4409 & 4338 & 4292 & 4237 & 4223 \\
125 & 4342 & 4142 & 4011 & 3473 & 3445 & 3437 \\
150 & 3723 & 3469 & 3218 & 3139 & 3074 & 3035 \\
\hline
\end{tabular}

Table 5.1: Events remaining after cuts applied to the $\eta=0.05$ dataset. 


\begin{tabular}{||c|cccccc||}
\hline $\begin{array}{c}\text { Beam } \\
\text { Energy }\end{array}$ & $\begin{array}{c}\text { Tracking } \\
\text { Cut }\end{array}$ & $\begin{array}{c}\phi \\
\text { Crack }\end{array}$ & $\begin{array}{c}\phi \\
\text { Upstream }\end{array}$ & $\begin{array}{c}\eta \\
\text { Upstream }\end{array}$ & $\begin{array}{c}\pi, \mu \\
\text { Cut }\end{array}$ & $\begin{array}{c}10 \sigma \\
\text { Energy Cut }\end{array}$ \\
\hline 10 & 2114 & 1605 & 1554 & 1515 & 1499 & 1493 \\
15 & 2373 & 1886 & 1837 & 1621 & 1618 & 1612 \\
20 & 6073 & 4902 & 4559 & 4527 & 4522 & 4504 \\
25 & 6390 & 5848 & 5356 & 5271 & 5266 & 5257 \\
30 & 6289 & 5801 & 5315 & 5236 & 5219 & 5208 \\
40 & 6382 & 5917 & 5644 & 5504 & 5494 & 5494 \\
50 & 6493 & 6308 & 6089 & 6014 & 5998 & 5992 \\
75 & 6336 & 6010 & 5968 & 5888 & 5864 & 5852 \\
100 & 6225 & 6157 & 6144 & 5988 & 5946 & 5936 \\
125 & 4195 & 4108 & 3822 & 3693 & 3644 & 3630 \\
150 & 3954 & 3841 & 3835 & 3591 & 3508 & 3484 \\
\hline
\end{tabular}

Table 5.2: Events remaining after cuts applied to the $\eta=0.45$ dataset.

\begin{tabular}{||c|cccccc||}
\hline $\begin{array}{c}\text { Beam } \\
\text { Energy }\end{array}$ & $\begin{array}{c}\text { Tracking } \\
\text { Cut }\end{array}$ & $\begin{array}{c}\phi \\
\text { Crack }\end{array}$ & $\begin{array}{c}\phi \\
\text { Upstream }\end{array}$ & $\begin{array}{c}\eta \\
\text { Upstream }\end{array}$ & $\begin{array}{c}\pi, \mu \\
\text { Cut }\end{array}$ & $\begin{array}{c}10 \sigma \\
\text { Energy Cut }\end{array}$ \\
\hline 10 & 1876 & 1490 & 1349 & 1295 & 1287 & 1165 \\
15 & 1907 & 1586 & 1391 & 1107 & 1104 & 1058 \\
20 & 2388 & 2042 & 1737 & 1436 & 1421 & 1346 \\
25 & 2551 & 2338 & 1911 & 1799 & 1799 & 1789 \\
30 & 2368 & 2205 & 1765 & 1621 & 1621 & 1616 \\
40 & 2602 & 2436 & 2093 & 2023 & 2023 & 2020 \\
50 & 2148 & 2058 & 1838 & 1762 & 1759 & 1754 \\
75 & 2148 & 2040 & 1996 & 1919 & 1915 & 1911 \\
100 & 2300 & 2287 & 2253 & 2185 & 2181 & 2162 \\
150 & 2444 & 2415 & 2409 & 2297 & 2291 & 2254 \\
\hline
\end{tabular}

Table 5.3: Events remaining after cuts applied to the $\eta=1.05$ dataset. 


\begin{tabular}{||c|ccccc||}
\hline Energy & $\begin{array}{c}\text { Total } \\
\text { Events }\end{array}$ & $\begin{array}{c}\phi \\
\text { Crack }\end{array}$ & $\begin{array}{c}\phi \\
\text { Upstream }\end{array}$ & $\begin{array}{c}\eta \\
\text { Upstream }\end{array}$ & $\begin{array}{c}\pi \\
\text { Cut }\end{array}$ \\
\hline 10 & 500 & 482 & 482 & 482 & 481 \\
25 & 500 & 490 & 490 & 490 & 490 \\
50 & 500 & 494 & 494 & 494 & 494 \\
75 & 537 & 531 & 531 & 531 & 531 \\
100 & 527 & 527 & 527 & 527 & 527 \\
125 & 520 & 513 & 513 & 513 & 511 \\
150 & 510 & 505 & 505 & 505 & 504 \\
\hline
\end{tabular}

Table 5.4: Events remaining after cuts for the Monte Carlo data at $\eta=0.05$.

\begin{tabular}{||c|ccccc||}
\hline Energy & $\begin{array}{c}\text { Total } \\
\text { Events }\end{array}$ & $\begin{array}{c}\phi \\
\text { Crack }\end{array}$ & $\begin{array}{c}\phi \\
\text { Upstream }\end{array}$ & $\begin{array}{c}\eta \\
\text { Upstream }\end{array}$ & $\begin{array}{c}\pi \\
\text { Cut }\end{array}$ \\
\hline 10 & 500 & 493 & 493 & 493 & 493 \\
25 & 512 & 511 & 511 & 511 & 511 \\
50 & 647 & 644 & 644 & 644 & 643 \\
75 & 520 & 517 & 517 & 517 & 517 \\
100 & 499 & 497 & 497 & 497 & 497 \\
125 & 505 & 502 & 502 & 502 & 502 \\
150 & 508 & 507 & 507 & 507 & 506 \\
\hline
\end{tabular}

Table 5.5: Events remaining after cuts for the Monte Carlo data at $\eta=0.45$.

\begin{tabular}{||c|ccccc||}
\hline Energy & $\begin{array}{c}\text { Total } \\
\text { Events }\end{array}$ & $\begin{array}{c}\phi \\
\text { Crack }\end{array}$ & $\begin{array}{c}\phi \\
\text { Upstream }\end{array}$ & $\begin{array}{c}\eta \\
\text { Upstream }\end{array}$ & $\begin{array}{c}\pi \\
\text { Cut }\end{array}$ \\
\hline 10 & 500 & 497 & 496 & 492 & 492 \\
25 & 500 & 499 & 499 & 499 & 499 \\
50 & 507 & 505 & 505 & 505 & 505 \\
75 & 511 & 482 & 482 & 482 & 482 \\
100 & 601 & 599 & 599 & 599 & 599 \\
125 & 509 & 507 & 507 & 507 & 507 \\
150 & 511 & 509 & 509 & 509 & 509 \\
\hline
\end{tabular}

Table 5.6: Events remaining after cuts for the Monte Carlo data at $\eta=1.05$. 


\begin{tabular}{||c|ccc|cc||}
\hline $\begin{array}{c}\text { Beam } \\
\text { Energy }\end{array}$ & $\chi_{\text {dof }}^{2}$ & $\begin{array}{c}\text { Response } \\
(\mathrm{GeV})\end{array}$ & $\begin{array}{c}\text { Resolution } \\
(\sigma / \mu)\end{array}$ & $\begin{array}{c}\text { Noise } \\
(\mathrm{MeV})\end{array}$ & $\begin{array}{c}\text { Resolution } \\
\sqrt{\left(\frac{\sigma}{\mu}\right)^{2}-\frac{N^{2}}{E^{2}}}\end{array}$ \\
\hline 10 & 1.05 & $9.644 \pm .010$ & $.0575 \pm .0008$ & 353 & $.0453 \pm .0014$ \\
15 & 1.09 & $14.490 \pm .013$ & $.0444 \pm .0007$ & 353 & $.0377 \pm .0010$ \\
20 & 1.12 & $19.436 \pm .012$ & $.0359 \pm .0005$ & 398 & $.0299 \pm .0007$ \\
25 & 1.31 & $24.177 \pm .011$ & $.0325 \pm .0004$ & 398 & $.0283 \pm .0005$ \\
30 & 1.17 & $29.136 \pm .013$ & $.0291 \pm .0003$ & 389 & $.0261 \pm .0005$ \\
40 & 1.68 & $38.953 \pm .014$ & $.0251 \pm .0003$ & 460 & $.0223 \pm .0004$ \\
50 & 0.87 & $48.883 \pm .029$ & $.0219 \pm .0004$ & 539 & $.0191 \pm .0005$ \\
75 & 0.74 & $73.301 \pm .024$ & $.0167 \pm .0003$ & 386 & $.0159 \pm .0003$ \\
100 & 1.18 & $98.147 \pm .040$ & $.0149 \pm .0002$ & 438 & $.0142 \pm .0002$ \\
125 & 1.22 & $122.920 \pm .028$ & $.0130 \pm .0002$ & 404 & $.0126 \pm .0002$ \\
150 & 1.44 & $147.000 \pm .032$ & $.0120 \pm .0002$ & 404 & $.0117 \pm .0002$ \\
\hline
\end{tabular}

Table 5.7: Response and resolution of the final datasets for $\eta=0.05$.

\begin{tabular}{||c|ccc|cc||}
\hline $\begin{array}{c}\text { Beam } \\
\text { Energy }\end{array}$ & $\chi_{\text {dof }}^{2}$ & $\begin{array}{c}\text { Response } \\
(\mathrm{GeV})\end{array}$ & $\begin{array}{c}\text { Resolution } \\
(\sigma / \mu)\end{array}$ & $\begin{array}{c}\text { Noise } \\
(\mathrm{MeV})\end{array}$ & $\begin{array}{c}\text { Resolution } \\
\sqrt{\left(\frac{\sigma}{\mu}\right)^{2}-\frac{N^{2}}{E^{2}}}\end{array}$ \\
\hline 10 & 1.23 & $9.764 \pm .016$ & $.0625 \pm .0013$ & 414 & $.0468 \pm .0025$ \\
15 & 1.05 & $14.778 \pm .018$ & $.0478 \pm .0009$ & 409 & $.0393 \pm .0013$ \\
20 & 1.31 & $19.903 \pm .011$ & $.0379 \pm .0004$ & 419 & $.0316 \pm .0006$ \\
25 & 1.58 & $24.870 \pm .012$ & $.0355 \pm .0004$ & 408 & $.0293 \pm .0006$ \\
30 & 1.07 & $29.824 \pm .013$ & $.0304 \pm .0003$ & 377 & $.0277 \pm .0005$ \\
40 & 1.50 & $39.780 \pm .013$ & $.0248 \pm .0002$ & 410 & $.0226 \pm .0003$ \\
50 & $\mathbf{0 . 7 7}$ & $49.716 \pm .017$ & $.0221 \pm .0002$ & 389 & $.0207 \pm .0002$ \\
75 & 1.64 & $74.470 \pm .017$ & $.0177 \pm .0002$ & 394 & $.0169 \pm .0002$ \\
100 & 1.05 & $99.213 \pm .020$ & $.0154 \pm .0002$ & 406 & $.0149 \pm .0002$ \\
125 & 1.33 & $124.070 \pm .028$ & $.0135 \pm .0002$ & 536 & $.0128 \pm .0002$ \\
150 & 1.08 & $149.130 \pm .032$ & $.0125 \pm .0002$ & 445 & $.0121 \pm .0002$ \\
\hline
\end{tabular}

Table 5.8: Response and resolution of the final datasets for $\eta=0.45$. 


\begin{tabular}{||c|ccc|cc||}
\hline $\begin{array}{c}\text { Beam } \\
\text { Energy }\end{array}$ & $\chi_{\text {dof }}^{2}$ & $\begin{array}{c}\text { Response } \\
(\mathrm{GeV})\end{array}$ & $\begin{array}{c}\text { Resolution } \\
(\sigma / \mu)\end{array}$ & $\begin{array}{c}\text { Noise } \\
(\mathrm{MeV})\end{array}$ & $\begin{array}{c}\text { Resolution } \\
\sqrt{\left(\frac{\sigma}{\mu}\right)^{2}-\frac{N^{2}}{E^{2}}}\end{array}$ \\
\hline 10 & 1.76 & $9.783 \pm .019$ & $.0653 \pm .0015$ & 281 & $.0589 \pm .0018$ \\
15 & 1.27 & $14.882 \pm .026$ & $.0546 \pm .0013$ & 271 & $.0515 \pm .0014$ \\
20 & 1.38 & $20.006 \pm .025$ & $.0444 \pm .0011$ & 311 & $.0416 \pm .0012$ \\
25 & 0.93 & $25.325 \pm .023$ & $.0377 \pm .0008$ & 273 & $.0361 \pm .0009$ \\
30 & 1.01 & $30.481 \pm .025$ & $.0331 \pm .0006$ & 252 & $.0320 \pm .0006$ \\
40 & 0.85 & $40.768 \pm .027$ & $.0290 \pm .0004$ & 336 & $.0278 \pm .0004$ \\
50 & 1.04 & $51.013 \pm .033$ & $.0267 \pm .0005$ & 280 & $.0261 \pm .0005$ \\
75 & 1.07 & $76.620 \pm .037$ & $.0210 \pm .0004$ & 275 & $.0207 \pm .0004$ \\
100 & 1.03 & $102.310 \pm .043$ & $.0187 \pm .0003$ & 281 & $.0185 \pm .0003$ \\
125 & 1.16 & $127.540 \pm .050$ & $.0173 \pm .0003$ & 266 & $.0172 \pm .0003$ \\
150 & 1.06 & $153.080 \pm .048$ & $.0159 \pm .0002$ & 331 & $.0157 \pm .0002$ \\
\hline
\end{tabular}

Table 5.9: Response and resolution of the final datasets for $\eta=1.05$.

\begin{tabular}{||c|cc||}
\hline Energy & $\begin{array}{c}\text { Response } \\
(\mathrm{GeV})\end{array}$ & $\begin{array}{c}\text { Resolution } \\
(\sigma / \mu)\end{array}$ \\
\hline 10 & $10.192 \pm .021$ & $.0431 \pm .0015$ \\
25 & $25.064 \pm .031$ & $.0261 \pm .0009$ \\
50 & $50.076 \pm .048$ & $.0177 \pm .0006$ \\
75 & $74.879 \pm .060$ & $.0161 \pm .0006$ \\
100 & $99.794 \pm .063$ & $.0134 \pm .0005$ \\
125 & $124.590 \pm .077$ & $.0130 \pm .0006$ \\
150 & $149.530 \pm .088$ & $.0124 \pm .0005$ \\
\hline
\end{tabular}

Table 5.10: Response and resolution of the Monte Carlo data at $\eta=0.05$.

\begin{tabular}{||c|cc||}
\hline Energy & $\begin{array}{c}\text { Response } \\
(\mathrm{GeV})\end{array}$ & $\begin{array}{c}\text { Resolution } \\
(\sigma / \mu)\end{array}$ \\
\hline 10 & $9.902 \pm .021$ & $.0436 \pm .0016$ \\
25 & $24.829 \pm .031$ & $.0264 \pm .0010$ \\
50 & $49.662 \pm .040$ & $.0192 \pm .0007$ \\
75 & $74.439 \pm .063$ & $.0164 \pm .0007$ \\
100 & $99.287 \pm .073$ & $.0135 \pm .0006$ \\
125 & $123.830 \pm .081$ & $.0137 \pm .0005$ \\
150 & $148.440 \pm .100$ & $.0127 \pm .0006$ \\
\hline
\end{tabular}

Table 5.11: Response and resolution of the Monte Carlo data at $\eta=0.45$. 


\begin{tabular}{||c|cc||}
\hline Energy & $\begin{array}{c}\text { Response } \\
(\mathrm{GeV})\end{array}$ & $\begin{array}{c}\text { Resolution } \\
(\sigma / \mu)\end{array}$ \\
\hline 10 & $9.558 \pm .027$ & $.0551 \pm .0020$ \\
25 & $24.132 \pm .038$ & $.0339 \pm .0013$ \\
50 & $48.195 \pm .051$ & $.0229 \pm .0009$ \\
75 & $72.383 \pm .067$ & $.0190 \pm .0008$ \\
100 & $96.337 \pm .069$ & $.0167 \pm .0005$ \\
125 & $120.440 \pm .084$ & $.0145 \pm .0007$ \\
150 & $144.440 \pm .098$ & $.0146 \pm .0006$ \\
\hline
\end{tabular}

Table 5.12: Response and resolution of the Monte Carlo data at $\eta=1.05$.

\begin{tabular}{||l|cccc||}
\hline Data Set & $\chi_{\text {dof }}^{2}$ & $\mathrm{C}$ & $\mathrm{S}$ & $\mathrm{N}$ \\
\hline$\eta=0.05$ & & & & \\
Monte Carlo & 0.93 & $.0081 \pm .0012$ & $.1105 \pm .0088$ & $.244 \pm .049$ \\
Monte Carlo $(\mathrm{N}=0)$ & 1.88 & $.0056 \pm .0011$ & $.1275 \pm .0035$ & - \\
Data & 1.91 & $.0000 \pm .0016$ & $.1443 \pm .0012$ & $.355 \pm .013$ \\
Noise Subtracted Data & 1.40 & $.0035 \pm .0010$ & $.1356 \pm .0032$ & $.152 \pm .052$ \\
Noise Subtracted Data & & & & \\
(N=0) & 1.43 & $.0019 \pm .0013$ & $.1399 \pm .0015$ & - \\
\hline$\eta=0.45$ & & & & \\
Monte Carlo & 0.79 & $.0079 \pm .0014$ & $.1189 \pm .0096$ & $.207 \pm .085$ \\
Monte Carlo (N =0) & 1.10 & $.0062 \pm .0012$ & $.1310 \pm .0038$ & - \\
Data & 1.39 & $.0040 \pm .0010$ & $.1403 \pm .0032$ & $.440 \pm .021$ \\
Noise Subtracted Data & 2.01 & $.0019 \pm .0024$ & $.1450 \pm .0039$ & $.067 \pm .189$ \\
Noise Subtracted Data & & & & \\
(N=0) & 1.79 & $.0016 \pm .0017$ & $.1456 \pm .0014$ & - \\
\hline$\eta=1.05$ & & & & \\
Monte Carlo & 0.59 & $.0061 \pm .0032$ & $.1535 \pm .0103$ & $.262 \pm .099$ \\
Monte Carlo (N =0) & $\mathbf{0 . 9 9}$ & $.0010 \pm .0114$ & $.1680 \pm .0060$ & - \\
Data & 1.89 & $.0082 \pm .0007$ & $.1638 \pm .0046$ & $.424 \pm .032$ \\
Noise Subtracted Data & 1.91 & $.0082 \pm .0007$ & $.1625 \pm .0044$ & $.328 \pm .042$ \\
Noise Subtracted Data & & & & \\
(N=0) & 3.22 & $.0057 \pm .0007$ & $.1776 \pm .0020$ & - \\
\hline
\end{tabular}

Table 5.13: Results of fits to $\frac{\sigma}{\mu}=\sqrt{C^{2}+\frac{S^{2}}{E}+\frac{N^{2}}{E^{2}}}$. 


\section{Chapter 6}

\section{$\eta$ Scan}

Understanding the uniformity of response of the calorimeter as a function of polar angle is imperative for extracting high precision electromagnetic physics results from this experiment. The uncertainty in energy measurements due to the intrinsic energy resolution of the detector can be eclipsed by systematic errors introduced by an unknown or poorly understood detector response. Since the intrinsic energy resolution of a $50 \mathrm{GeV}$ electron is typically about $2 \%$, it is necessary to understand the response function of the calorimeter to a level that is at least as good. In this chapter, we will explore the response as a function of $\eta$, or almost equivalently, as a function of angle of incidence.

\subsection{Testbeam Data Set}

The $\eta$ scan was performed using beams of $100 \mathrm{GeV}$ electrons striking the modules from a pseudorapidity of 0.05 to 0.95 . The data analysis in this study was done in an identical way as that of the energy scans (chapter 5). 


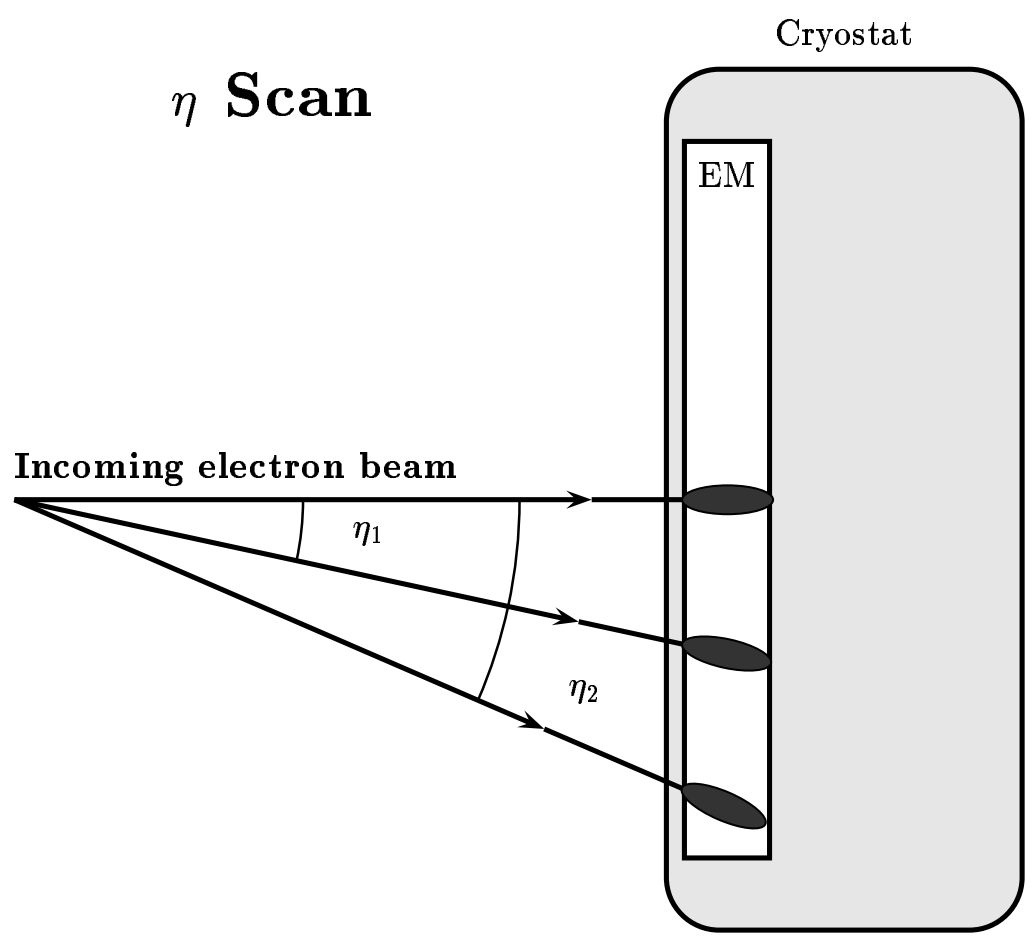

Figure 6.1: Beam projections into the testbeam cryostat for the $\eta$ scan. 


\begin{tabular}{||lc||}
\hline Cut & Cut \\
Name & Definition \\
\hline$\phi$ crack cut & \\
(upper module) & $31.3<\phi<31.7$ \\
(lower module) & $32.3<\phi<32.7$ \\
key notch cut & $0.015<\eta<0.035^{a}$ \\
\hline
\end{tabular}

Table 6.1: Tracking requirements imposed on the $\eta$ scan data set.

${ }^{a}$ for $\eta \sim 0.05$

The tracking and signal gain correction packages (TB90L2_SURVEY[25] and TB90L2_GAINCOR[26]) were used. Tracking requirements were made to enable reliable momentum corrections and to remove events whose tracks were close to module structural components (Tab. 6.1).

The resulting energy distributions were then fitted using a gaussian function. Two scans were conducted, one in each of the interior CCEM modules. The response was determined by taking the average of the two scans fitted mean at each $\eta[27]$.

\subsection{Monte Carlo Data Set}

The Monte Carlo was used to recreate this scan. Simulated runs were done covering the pseudorapidity range of 0.05 to 1.05 in steps of 0.10 , each with 500 events. As in the energy scan study, few events were removed when tracking requirements were imposed. The energy depositions in each of the four electromagnetic layers and the first fine hadronic layer were weighted using the same weights as were used for the data. A gaussian was then fitted 


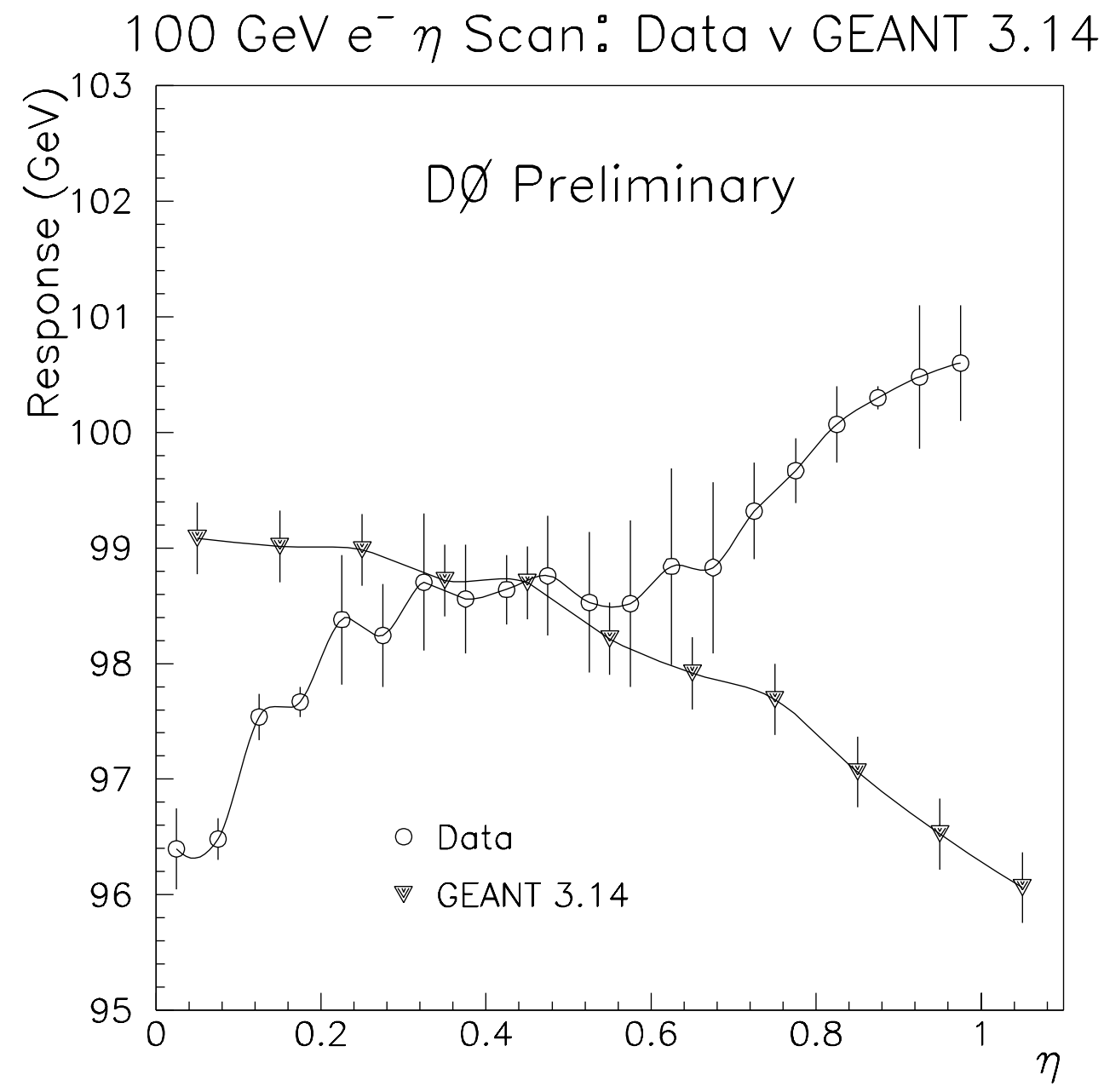

Figure 6.2: Response vs. $\eta$ for $100 \mathrm{GeV}$ electrons for both the testbeam data and the simulation. 
to the energy distributions. The simulated response was then normalized to the testbeam data at $\eta=0.45$.

\subsection{Comparison of Monte Carlo and Data}

The results from both the testbeam data and the simulation are shown in Fig. 6.2. From the plot, we can see that the testbeam response increases by $4 \%$ from a pseudorapidity of 0.05 to 0.95 while the Monte Carlo predicts a $2.5 \%$ drop over the same region. The energy depositions in each layer are shown in Fig. 6.3 for both the data and the simulation. This figure shows that there appears to be a problem with the longitudinal shower shape. Although there is good agreement in layer 4 , layers 1 and 2 show systematic shifts between the two data sets. Layer 3 shows the worst agreement and accounts for most of the difference at small $\eta$. This discrepancy between the data and the simulation is very disturbing. Potentially, this could lead to large systematic errors if this Monte Carlo is used to extract a $\mathrm{W}^{ \pm}$mass from collider data. 

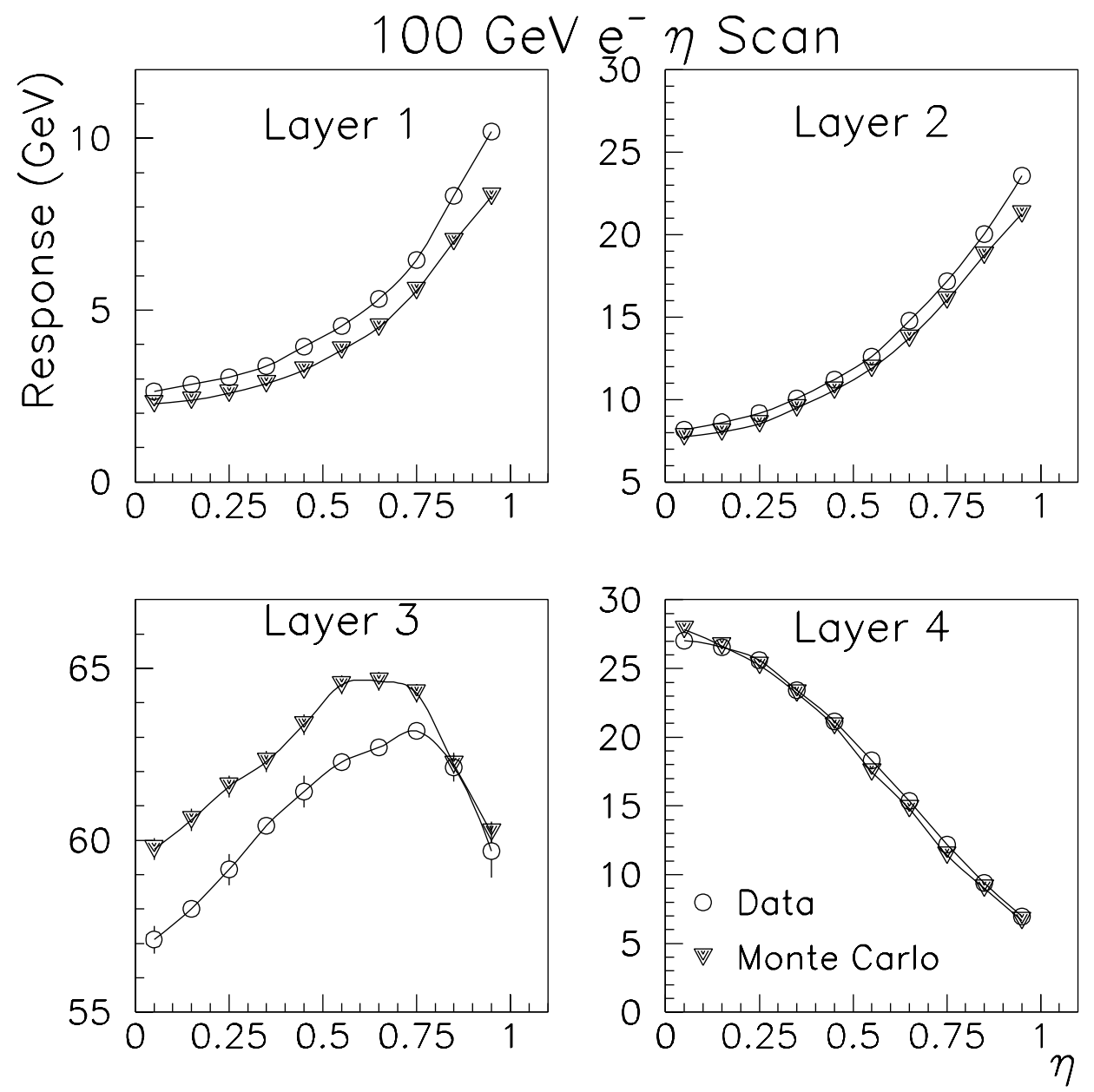

Figure 6.3: Energy deposition by layer for the four electromagnetic layers as a function of pseudorapidity for both testbeam data and the GEANT simulation. 


\section{Chapter 7}

\section{EGS4 - GEANT 3.14 Comparison}

The discrepancies between the Monte Carlo and the testbeam data presented in the last chapter are troubling. The inability of GEANT 3.14 to predict the behavior of the response of the calorimeter as a function of pseudorapidity can lead to problems making measurements in the collider environment. This concern prompted another study of this behavior, this time using the EGS4[28] simulation package[29]. Comparisons were made between EGS4 and GEANT 3.14 at $10 \mathrm{GeV}$ and also with the data at $100 \mathrm{GeV}$.

\subsection{Geometry}

The detector modeling aids in EGS4 are not as developed as those in GEANT. This made modeling the detector more difficult. However, since we were concerned with the response of the calorimeter in regions far away from structural components, most of them were left out. Essentially, a longitudinally correct model of the detector was created. 
The geometry used in this simulation is shown in Fig. 7.1. Although the upstream counters were left out, they account for only $0.15 X_{0}$ out of a total $1.3 X_{0}$ in front of the CCEM. Devices inside the testbeam cryostat such as the liquid argon excluder and the CC cryostat wall simulator were included as well as the cryostat itself. The CCEM included only the components inside the support box, essentially just the repeated unit cell structure (sec 3.2). This did include the individual uranium plates, argon gaps, signal and readout boards as well as the copper pads. In addition the front and back stainless steel plates were implemented. To ensure transverse shower containment, the lateral dimensions of the simulated modules were set at $40 \mathrm{~cm}$ transverse to the scan direction $(\phi)$ and $200 \mathrm{~cm}$ along it $(\eta)$. To provide longitudinal containment, the first layer (41 $X_{0}$ ) of the fine hadronic module was also modeled in the same fashion as the electromagnetic module. The same geometry was also developed in GEANT in order to make an accurate comparison of the two Monte Carlos.

\subsection{Results at $10 \mathrm{GeV}$}

An analogous study to that conducted in the testbeam at $100 \mathrm{GeV}$ was repeated at $10 \mathrm{GeV}$ for the two Monte Carlos. Ten $\mathrm{GeV}$ electrons were used to scan an $\eta$ region from 0.05 to 1.05 . Both simulations used $10 \mathrm{KeV}$ energy cutoffs. In the analysis for this data set, no attempt was made to correct for energy lost in the upstream material. The results are shown in Fig. 7.2.

The responses of the two simulations have been normalized to $10 \mathrm{GeV}$ at a pseudorapidity of 0.05 . It can be seen that although the two Monte Carlos 
Figure 7.1: Geometrical configuration used in the comparison of EGS4 and GEANT 3.14. 


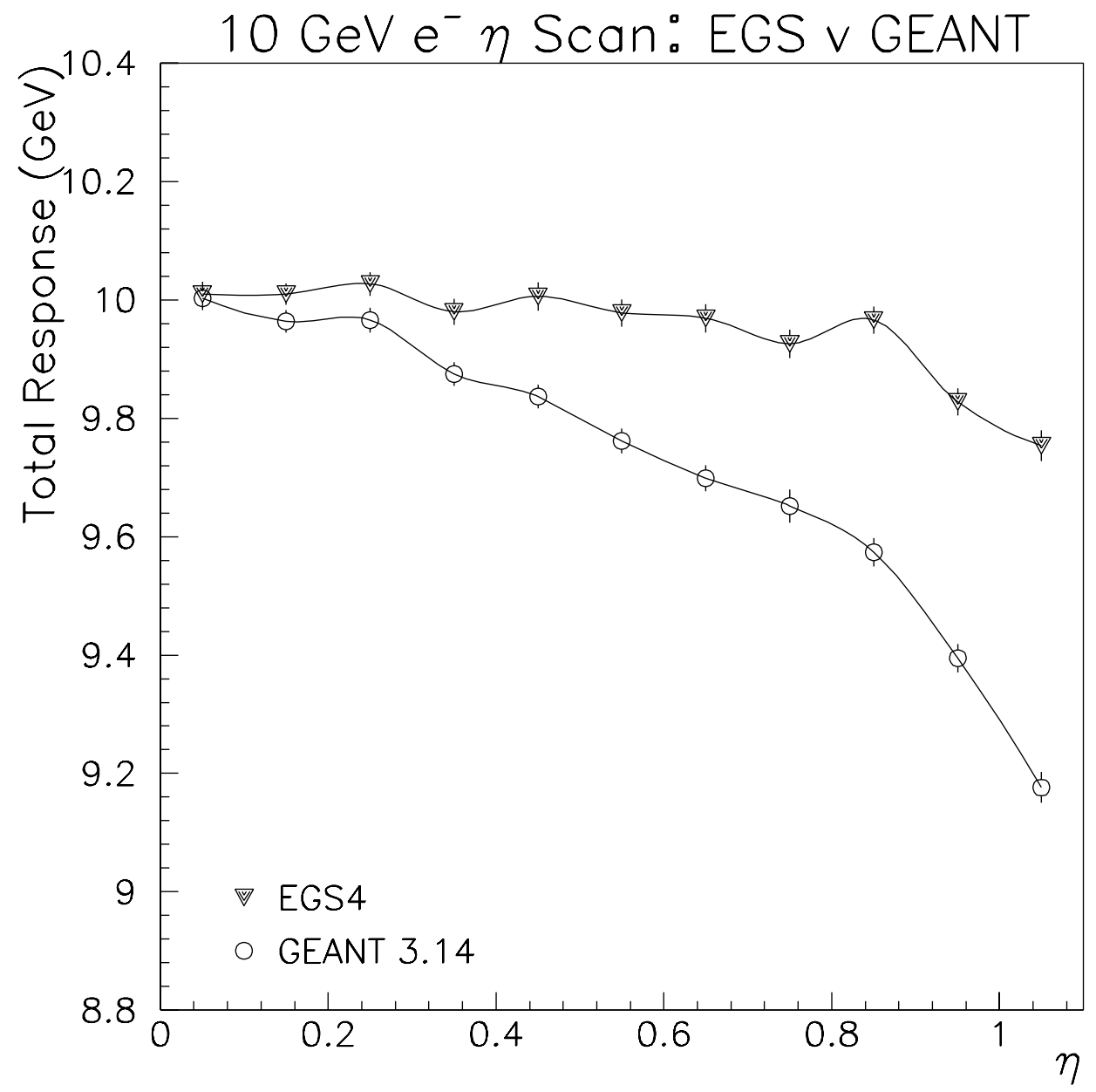

Figure 7.2: Results of the $\eta$ scan for both EGS4 and GEANT 3.14 using 10 $\mathrm{GeV}$ electrons. No upstream correction factor (22\%) was applied to layer 1. 
both show a drop in response as high angles of incidence, the magnitude of the GEANT drop is larger than that of EGS $(8.2 \%$ vs. $2.5 \%)$. The same trends are seen in the longitudinal shower development as a function of $\eta$, as shown in Fig. 7.3. Discrepancies appear at large $\eta$. Most of the difference shows up in the third layer where the largest fraction of the shower energy is deposited. To the level of $50 \mathrm{MeV}$, all the energy, both live and dead, is accounted for in both simulations. Compared to EGS, too little energy is being deposited in the liquid argon at large angles of incidence by GEANT.

\subsection{Results at $100 \mathrm{GeV}$}

There was a desire to determine which simulation best reproduced our testbeam data. As shown in chapter 6 , scans existed only for $100 \mathrm{GeV}$ electrons. A second study simulating $100 \mathrm{GeV}$ electrons was conducted using EGS for comparison to both the testbeam data and GEANT. Results are shown in Fig. 7.4.

The same algorithms were used to analyze all data sets. In this case, the upstream energy correction described in chapter 5 was used in all data sets. The comparison was made by normalizing the Monte Carlo responses to the testbeam results at $\eta=0.45$.

The two Monte Carlos show behavior at $100 \mathrm{GeV}$ similar to that at 10 $\mathrm{GeV}$. At low pseudorapidity their responses are similar, differing by at most 2 standard deviations. At high pseudorapidity the simulations diverge. At $\eta$ $=1.05$, EGS predicts a $1.5 \%$ increases in response while GEANT shows a $3 \%$ 

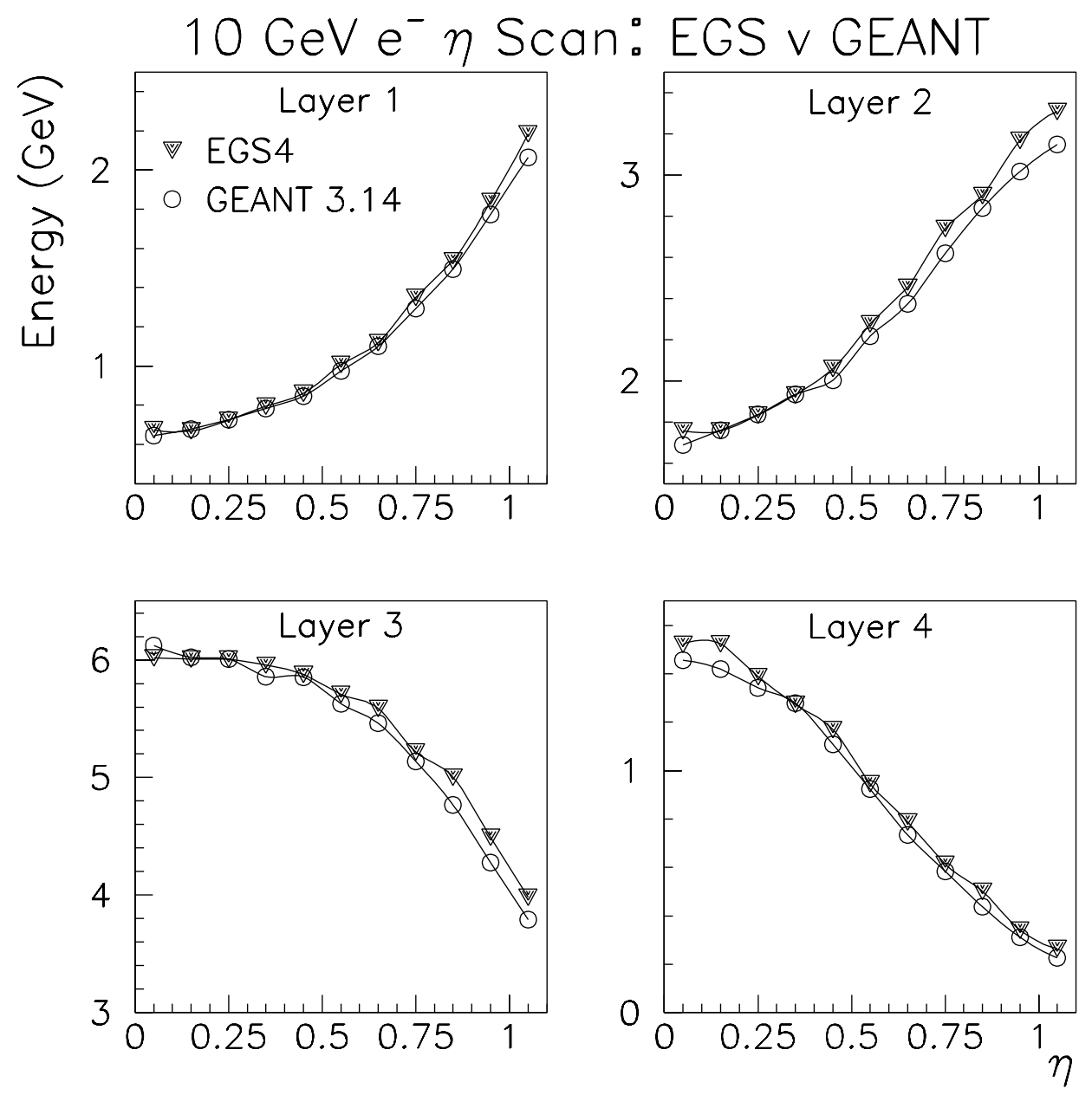

Figure 7.3: Comparison of EGS4 and GEANT 3.14 using $10 \mathrm{GeV}$ electrons. Results are plotted vs. $\eta$ for the four electromagnetic layers. 


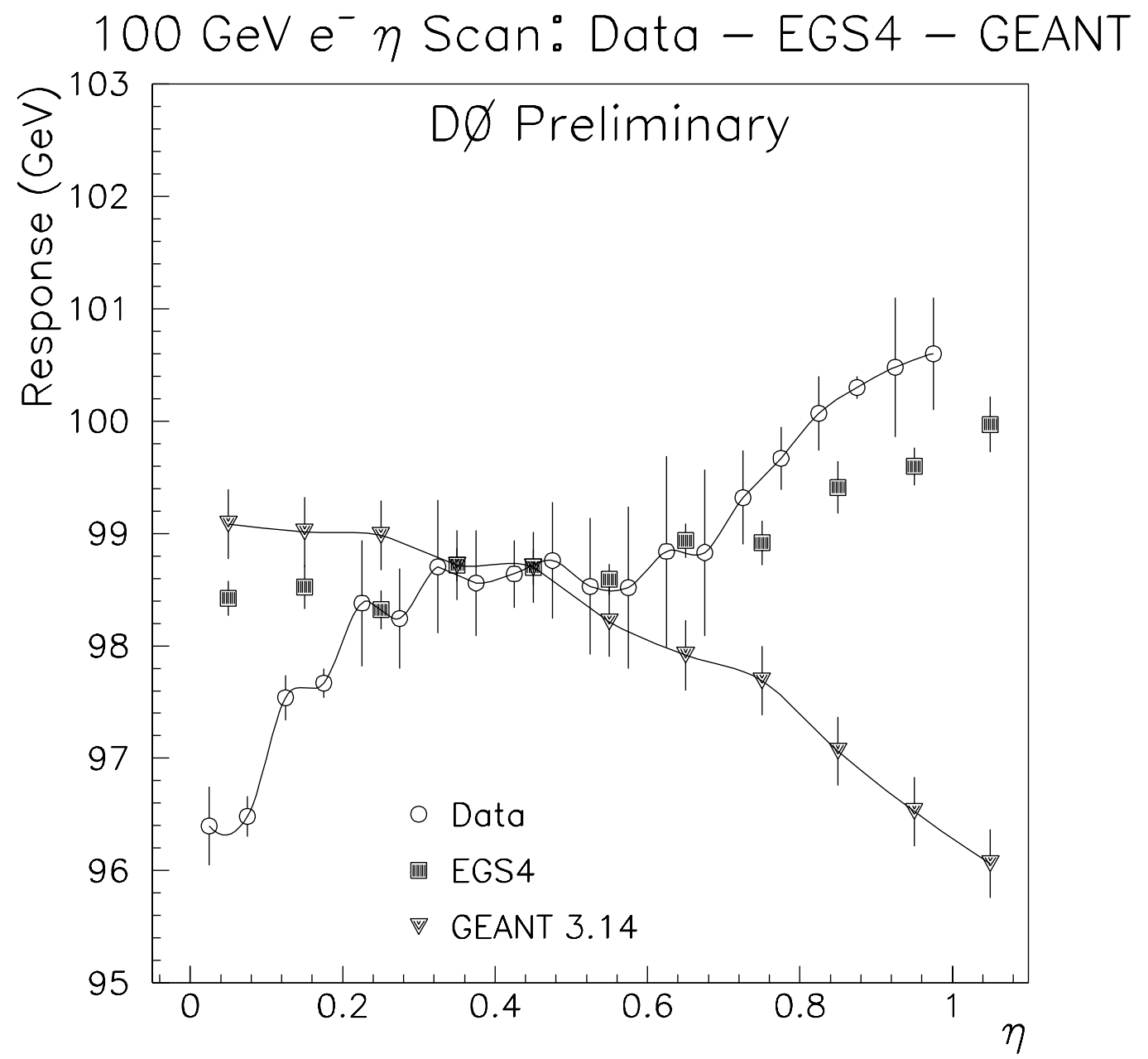

Figure 7.4: Results of the $100 \mathrm{GeV} \eta$ scan for testbeam data and both simulations. 
decrease in signal relative to $\eta=0.45$. The testbeam data shows a $2 \%$ increase in signal over the same range. Although not in complete agreement, the data more closely resembles EGS than GEANT.

\subsection{Results Using $\delta$ Rays}

One suggested source of the discrepancies seen between EGS and GEANT has been the treatment of $\delta$ rays[30]. These low energy electrons are emitted in the ionization process and have sufficient energy to cause ionization themselves. The default in GEANT 3.14 is to not track the secondary particles $(\delta$ rays) explicitly. Instead, the energy is deposited locally and subjected to an energy smearing based on the Landau distribution[22]. This can be overridden, however. GEANT can be told to explicitly track the secondaries down to the effective energy cutoff.

Since this was a concern, the study in section 7.2 was repeated. This time the $\delta$ rays were explicitly tracked down to $10 \mathrm{KeV}$. The results of all three data sets, EGS4, GEANT without $\delta$ rays, and GEANT with $\delta$ rays, are shown in Fig. 7.5.

As can be seen in the figure, explicit tracking of the $\delta$ rays does improve the agreement between EGS and GEANT. In this case, the GEANT response drops by only $4.2 \%$ compared to $8.2 \%$ with local energy deposition. This compares much better with the $2.5 \%$ drop predicted by EGS. This would imply that the mobility of the $\delta$ rays provides a net flux of energy out of the uranium plates and into the liquid argon. 


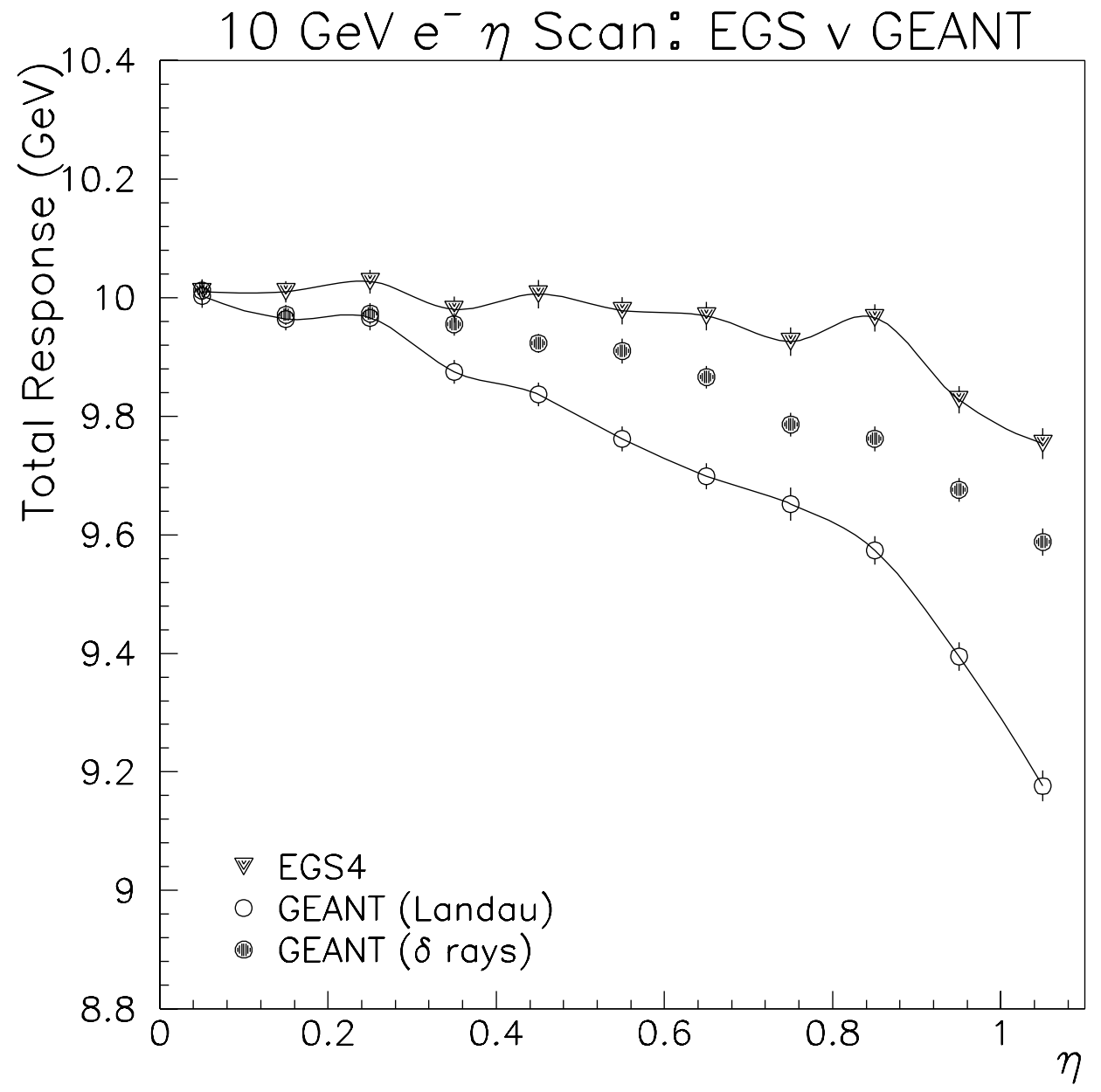

Figure 7.5: Comparison of EGS4 and GEANT 3.14. The delta rays in GEANT are explicitly tracked down to $10 \mathrm{KeV}$. 


\begin{tabular}{||ll||}
\hline Simulation & $e / \mu$ \\
\hline EGS4 & .624 \\
GEANT 3.14 (no $\delta$ rays) & .606 \\
GEANT 3.14 ( $\delta$ rays) & .616 \\
\hline
\end{tabular}

Table 7.1: Total live energies weighted by sampling fractions for the three Monte Carlo data sets.

Another difference between the two Monte Carlos can be seen in the predicted electron signal suppression, or the $e / \mu$ ratio (sec. 2.5). This suppression factor can be studied by taking the ratio of the total energy:

$$
E_{t o t}=\sum_{i=1}^{5} \beta_{i} E_{i}
$$

where the $\beta_{i}$ are the inverse of the individual layer sampling fractions and $E_{i}$ is the energy deposited in layer $i$, and the total incoming particle energy. These ratios are shown in Tab. 7.1 for the EGS data set and the two GEANT data sets. Although explicit tracking of $\delta$ rays improves the agreement between EGS and GEANT, both GEANT data sets have lower $e / \mu$ ratios compared to EGS. Previous studies of this sort[18] using EGS suggest that this ratio should not be highly dependent on the angle of incidence. This implies that aside from upstream energy losses, the response should be relatively uniform. Both EGS and the data see moderate increases in response as a function of angle when a correction for upstream looses is made while GEANT sees a larger decrease (Fig. 7.4). 


\section{Chapter 8}

\section{W Mass Measurement}

This chapter will apply the results from previous chapters in a study of the systematic bias induced in the determination of the $\mathrm{W}^{ \pm}$boson mass due to modeling the response of the calorimeter incorrectly. From section 2.2 , we saw that in order to make a successful measurement of the mass, the total error must be kept to $\sim 100 \mathrm{MeV}$. Since this mass determination (section 2.3) is dependent on both the simulation and behavior of the detector, these topics must be well understood. Although an effort was made to make the testbeam as identical to the full calorimeter as possible, differences exist. These differences include not only the upstream material in the beamline at the testbeam but also the lack of central detectors that are only part of the full detector. The Monte Carlo was intended to provide a connection between the collider environment and the testbeam. Reproduction of the testbeam data by the simulation would have built confidence that a detailed simulation of the full detector could be trusted. This simulation would then be used to study the resolution and response functions of the full detector. 
In chapter 5, we saw that the simulation based on GEANT predicted the resolution of the detector. However, in chapter 6 , we saw that significant discrepancies between the testbeam data and the simulation for the response as a function of pseudorapidity existed. Using the resolution measured in the detector, we will explore the biases caused by using the flawed response function to determine the $\mathrm{W}^{ \pm}$mass from collider data. This will also lead to insight into the size of the systematic errors that can be caused from a lack of understanding of the electromagnetic calorimeter's response function.

\subsection{Event Simulation}

As we discussed in section 2.3, several Monte Carlo data samples spanning the range of possible $\mathrm{W}^{ \pm}$masses are needed to arrive at a value for $M_{W}$ from collider data. The distributions for the transverse mass and the transverse momentum of the leptons derived from the simulated data samples are compared to the distributions from the collider data to arrive at a value for the mass. The production and decay of the boson in the collider environment must be modeled as well as the response of the detector to the decay products. This section will describe the method used to create these simulated data sets.

The event generation was modeled using the next to leading order calculation for $\mathrm{W}^{ \pm}$boson production done by Arnold and Kauffman[31]. They reduce the theoretical uncertainty in the cross section formula from $O\left(\alpha_{s}^{2} \ln ^{4}\left(1 / \alpha_{s}^{2}\right)\right)$ (the highest order terms left out of the calculation being of this form) to $O\left(\alpha_{s}^{2}\right)$ by matching the analytical forms from high $p_{T}$ (perturbative) to those at low 
$p_{T}$ (gluon resummation). The simulated bosons obey the resultant $d \sigma /\left(d p_{T} d y\right)$ distribution where $y$ is the rapidity:

$$
\begin{aligned}
y & \left.=1 / 2 \ln \left[\left(E+p_{L}\right) /\left(E-p_{L}\right)\right]\right) \\
& =\tanh ^{-1} \frac{p_{L}}{E} \\
& \simeq \eta
\end{aligned}
$$

and $p_{L}$ is the longitudinal component of the momentum. The bosons are then decayed into an electron and an electron neutrino. The recoiling hadrons were not explicitly created in this simulation.

The response of the detector to the decay products $\left(e, \nu_{e}\right)$ must be simulated. The method used to simulate the detector is that used by the UA2 collaboration at CERN[32][33][34]. The response of the detector to the electron is simulated explicitly while the hadronic response, and the subsequent measurement of the neutrino, is modeled in a more global sense. These smeared values for the decay product momenta are then used to calculate the kinematic variables $\left(m_{T}, p_{T}^{e}\right.$, and $\left.p_{T}^{\nu}\right)$ used in the fitting procedure.

To produce a realistic value for the electron momentum, the resolution of the detector as measured in the testbeam (chapter 5) was used. A resolution function (Eq. 5.1) with a sampling term $S$ equal to $14.5 \%$, a constant term $C$ equal to $0.4 \%$, and a noise term $N$ equal to $400 \mathrm{MeV}$ :

$$
\begin{aligned}
\sigma(E) & =\sqrt{C^{2} E^{2}+S^{2} E+N^{2}} \\
& =\sqrt{(0.004)^{2} E^{2}+(0.145)^{2} E+(0.400)^{2}}
\end{aligned}
$$

provides a value for the measurement uncertainty. The smeared energy of the 
electron, $E_{e}^{s}$, is defined to be:

$$
E_{e}^{s}=E_{e}+R \sigma\left(E_{e}\right)
$$

where $E_{e}$ is the unsmeared energy and $R$ is a random number obeying a gaussian distribution with a mean of 0 and sigma of 1 .

The angle between the electron momentum vector and the $\mathrm{W}^{ \pm}$momentum vector, $\phi^{e, W}$, is also smeared. The smearing function was obtained by simulating the electron tracks in the calorimeter using GEANT. Figures 8.1 and 8.2 show the resolutions of the smearing for both $\eta$ and $\phi$. The values used in the smearing are $\sigma_{\eta}=0.0113$ and $\sigma_{\phi}=0.01$ radians.

The smearing of the neutrino energy is more complicated. Since only the boson is created in the event generation without the recoiling hadrons, the $p_{T}$ of the $\mathrm{W}^{ \pm}$is used to model the neutrino detection. The underlying event in $\mathrm{W}^{ \pm}$candidate events (particles not associated with the $\mathrm{W}^{ \pm}$decay products) is analyzed to understand the dependence of the total transverse energy flow in the event as a function of the transverse momentum of the boson (this data sample to be described in the next section). Minimum bias and QCD (jet) data can then be used to determine the missing energy measurement resolution, $\sigma_{H_{T}}\left(E_{T}\right)$. This function is then used to smear the $p_{T}$ of the boson. Combined with smearing the angle between the electron and the boson and the smeared electron response, the smeared value of the $\mathbb{F}_{T}$ (the measurement of the neutrino) can be calculated.

To understand the underlying event, collider $\mathrm{W}^{ \pm}$candidates are separated into samples based on the $p_{T}$ of the boson. For each sample, a distribution 


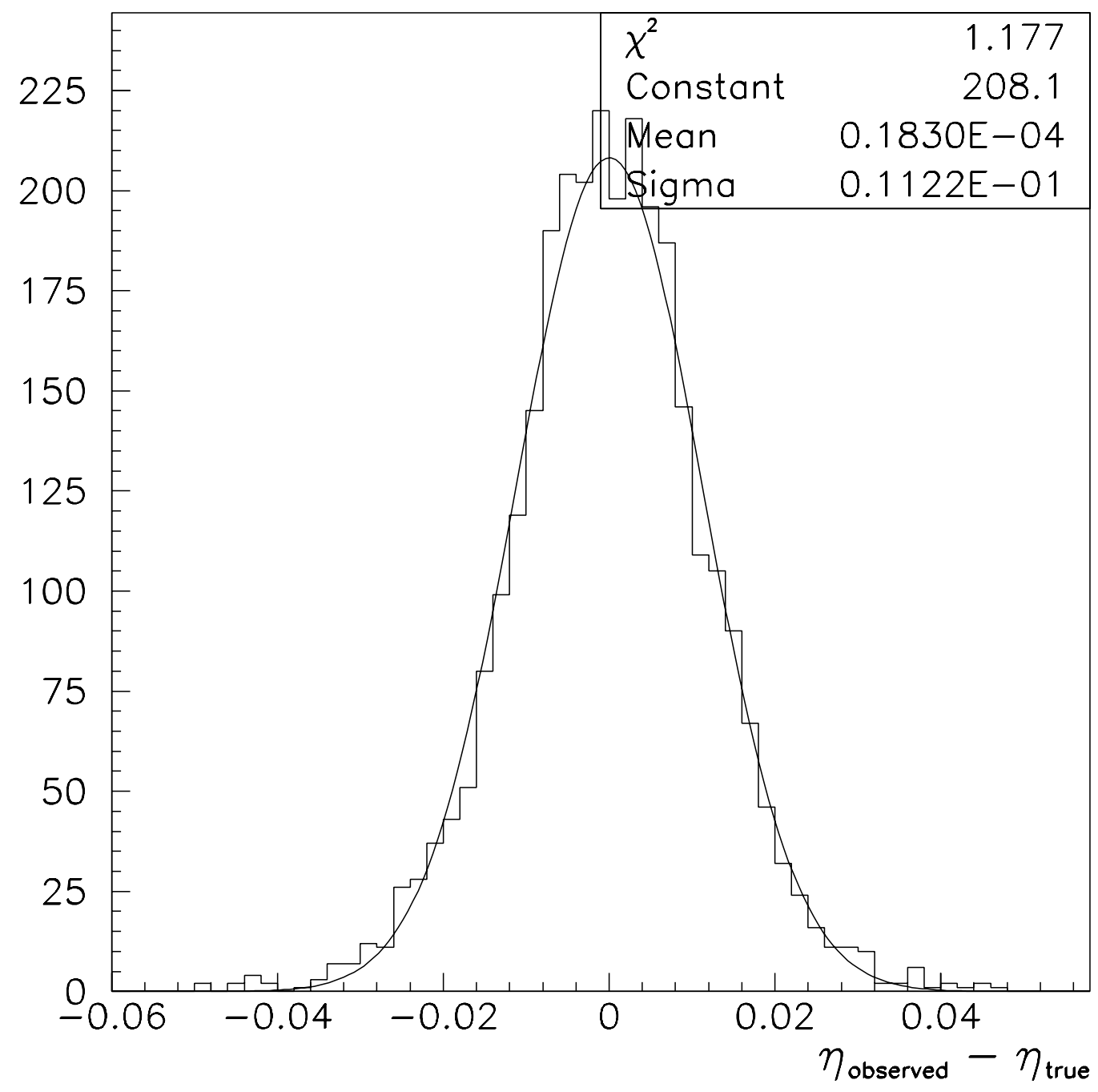

Figure 8.1: Distribution of the difference between the reconstructed electron $\eta$ and the true electron track. Results are from GEANT[35]. 


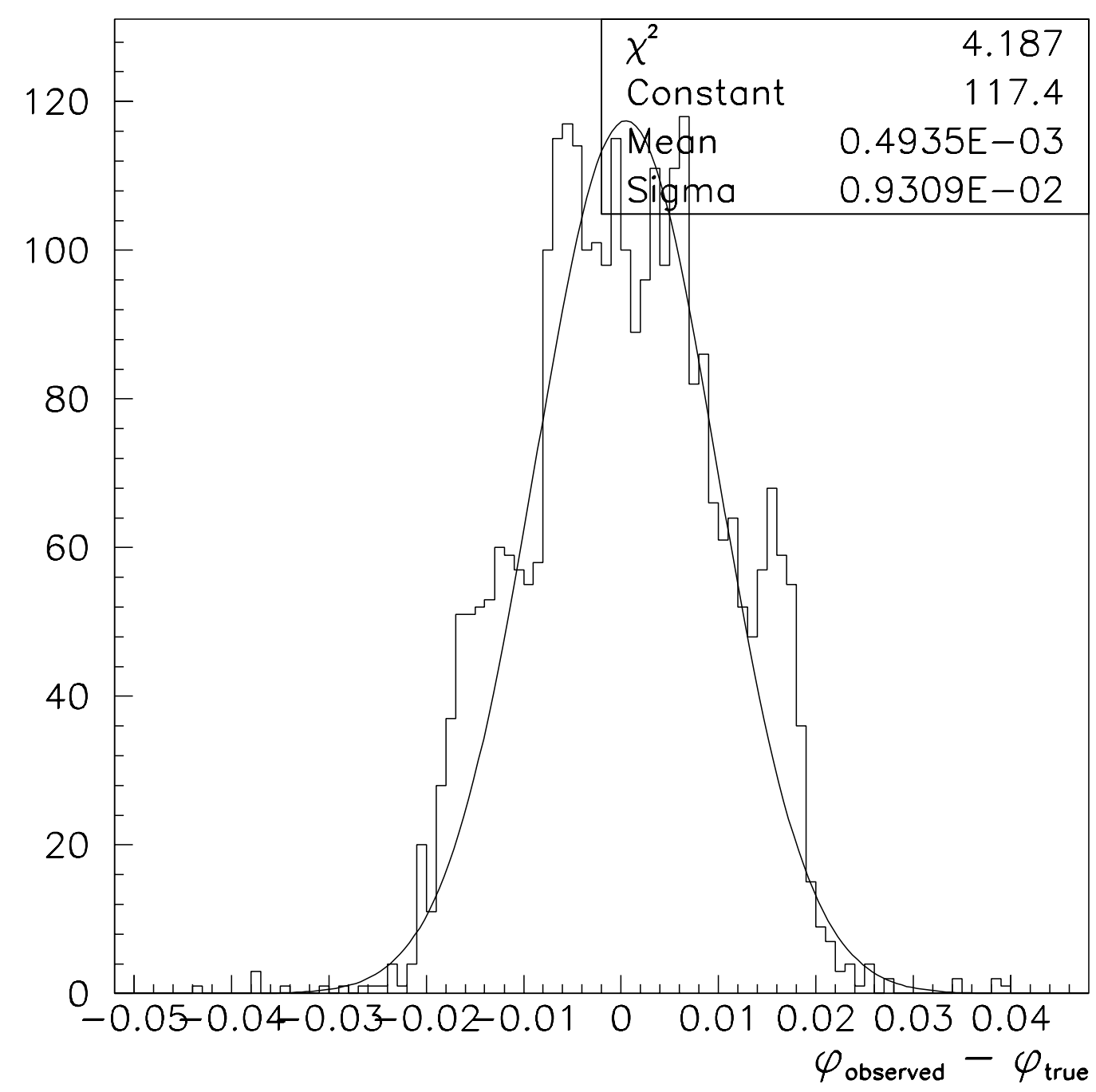

Figure 8.2: Distribution of the difference between the reconstructed electron $\phi$ angle and the true electron track. Results are from GEANT[35]. 
of the total scalar transverse energy, the sum of $(E \sin \theta)$ over all calorimeter cells except those assigned to the electron,

$$
E_{T}=\sum E \sin \theta-E_{T}^{e}
$$

is made. The mean $E_{T}$ for each distribution can be plotted against the mean $p_{T}$ of the boson for each sample to reveal the dependence of the hadronic activity of the event on the momentum of the boson (Fig. 8.3).

In addition, the jet behavior of $\mathrm{W}^{ \pm}$candidates was studied. Since the $\not_{T}$ resolution of the detector is different for minimum bias events and jet events, the neutrino measurement resolution for simulated $\mathrm{W}^{ \pm}$will depend on whether there is an accompanying jet in the event. Collider $\mathrm{W}^{ \pm}$events are used to calculate the probability of there being two and only two accompanying jets in the event. Two jets were required since monojet events are usually characterized by the loss of the second jet (it escapes the detection through detector inefficiencies), resulting in excessive amounts of $\not_{T}$ and a flawed resolution function. Two jet events offer a better representation of the detector's $H_{T}$ resolution since the particles with most of the $p_{T}$ of the event are detected. From this distribution and the mean $E_{T}$ derived earlier, the probability for the simulated event to have a jet is determined (Fig. 8.4)[35].

The measurement resolution of the components of the $\mathbb{E}_{T}$ has been examined using minimum bias and jet events from the detector. In minium bias events, few high energy neutrinos are produced that can escape the detector. In this case, it is assumed that any momentum imbalance is due to the energy measurement resolution of the detector. The $\mathrm{x}$ and $\mathrm{y}$ components are treated 


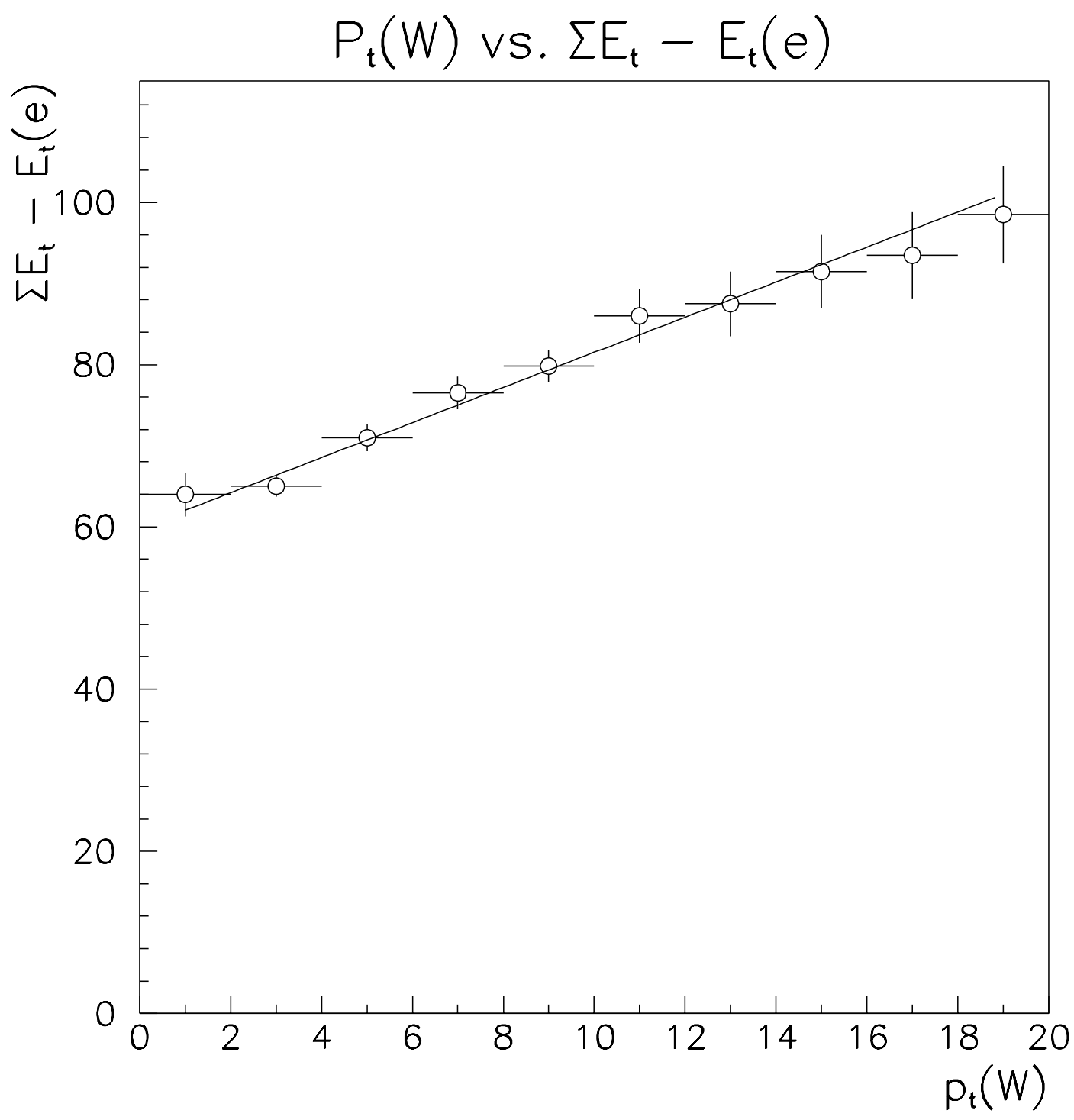

Figure 8.3: The mean $E_{T}$ minus the $E_{T}$ of the decay electron plotted vs. the $p_{T}$ of the $\mathrm{W}^{ \pm}$boson[35]. 


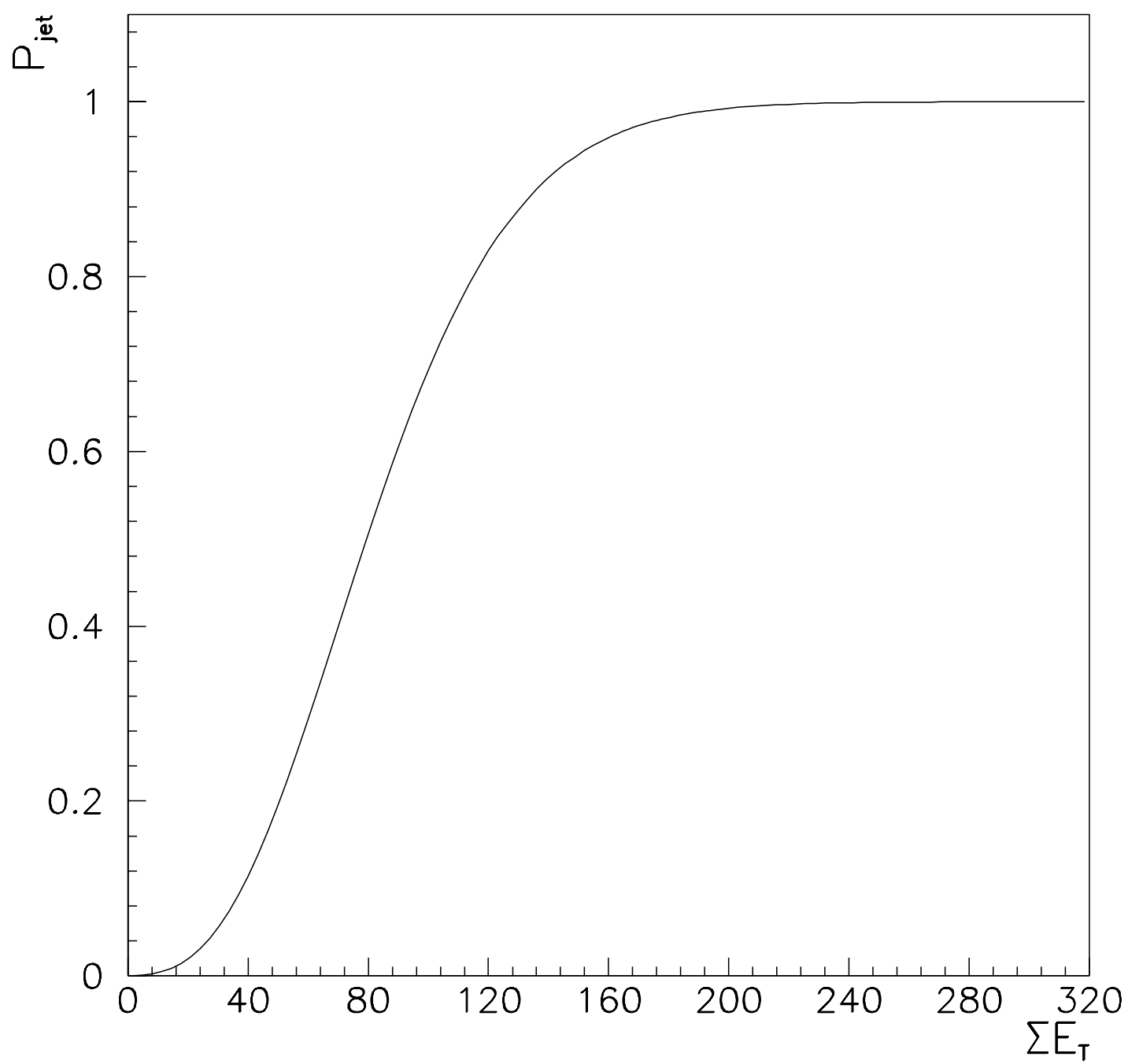

Figure 8.4: The probability of an event having two jets as a function of the sum of the $E_{T}$. 
separately and are seen to scale with $E_{T}$ (Fig. 8.5)[36]:

$$
\sigma_{x, y}=1.65 \mathrm{GeV}+0.029 E_{T}
$$

For QCD events, most of the momentum imbalance is due to mismeasuring one of the two jets. The components of the $\mathbb{H}_{T}$ resolution also scale with $E_{T}$ (Fig. 8.6)[35]:

$$
\sigma_{x, y}=1.28 \mathrm{GeV}+0.011 E_{T}
$$

The appropriate resolution function is used depending on whether the simulated event is found to have jet activity giving a more realistic neutrino measurement resolution for the entire range of $p_{T}(W)$.

Given these distributions, it is now possible to smear the transverse momentum components of the boson momentum. Using the true $p_{T}$ of the boson, we pick a value for the $E_{T}$ of the event from the distributions of $E_{T}$ binned by the $p_{T}$ of the boson. From this value, we obtain a resolution for the $\mathbb{E}_{T}$ which we use to smear the $p_{T}(W)$ :

$$
p_{T x, y}^{s}(W)=p_{T x, y}(W)+R \sigma_{\not_{T} x, y}
$$

where $p_{T}^{s}(W)$ is the smeared momentum.

Using these smeared quantities, we can now calculate the transverse mass and the transverse momentum components of the two decay leptons. The transverse momentum of the electron was smeared explicitly. The transverse momentum of the neutrino comes from the smeared values of $p_{T}(W)$ and $p_{T}(e)$ :

$$
\begin{aligned}
p_{T}^{s}(\nu) & =F\left(p_{T}^{s}(W), p_{T}^{s}(e)\right) \\
& =\sqrt{p_{T}^{s 2}(W)+p_{T}^{s 2}(e)-2 p_{T}^{s}(W) p_{T}^{s}(e) \cos \phi^{e, W}}
\end{aligned}
$$


$D \varnothing$ preliminary

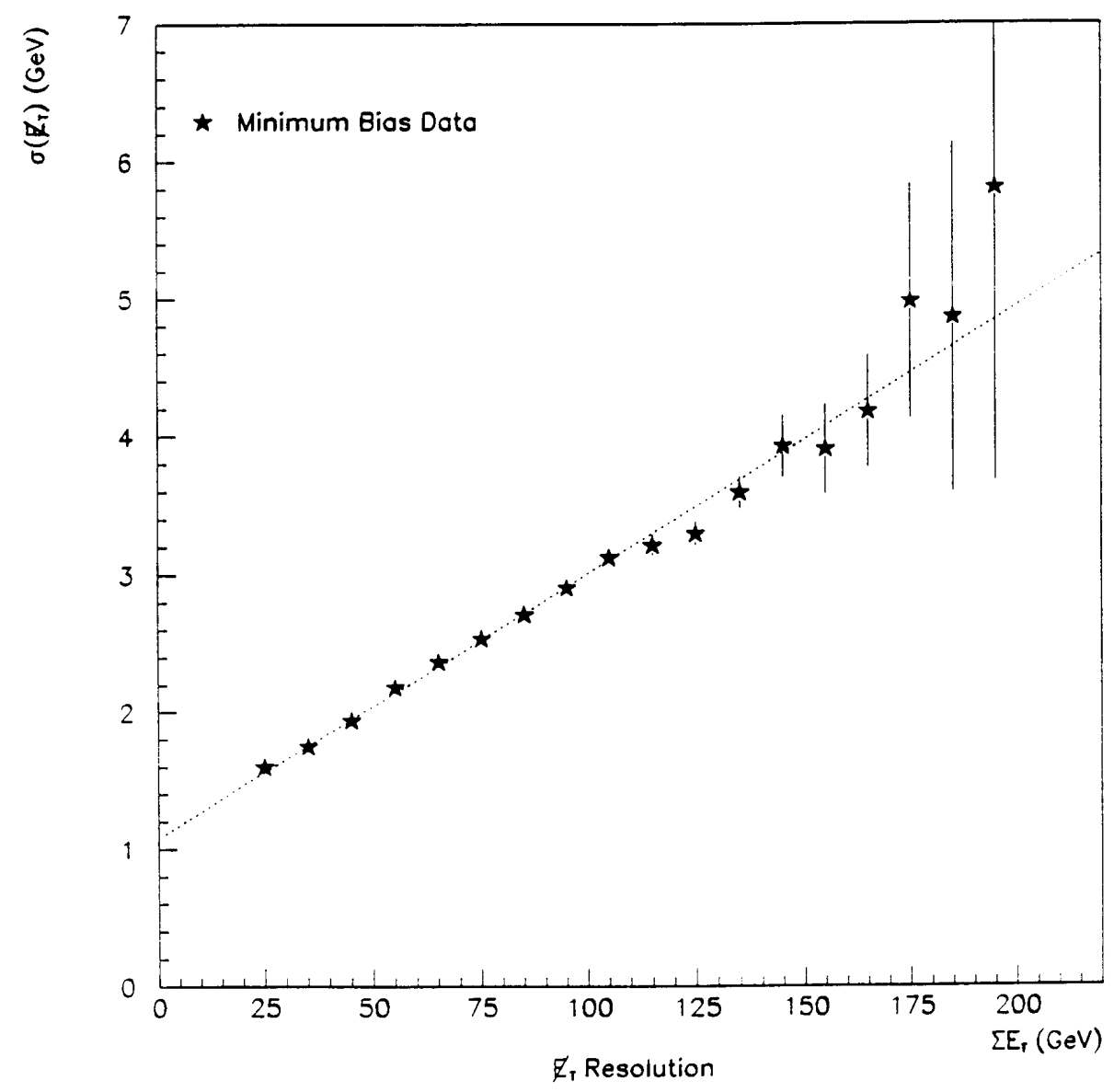

Figure 8.5: $E_{T}$ resolution as a function of total scalar $E_{t}$ as derived from minimum bias events. 


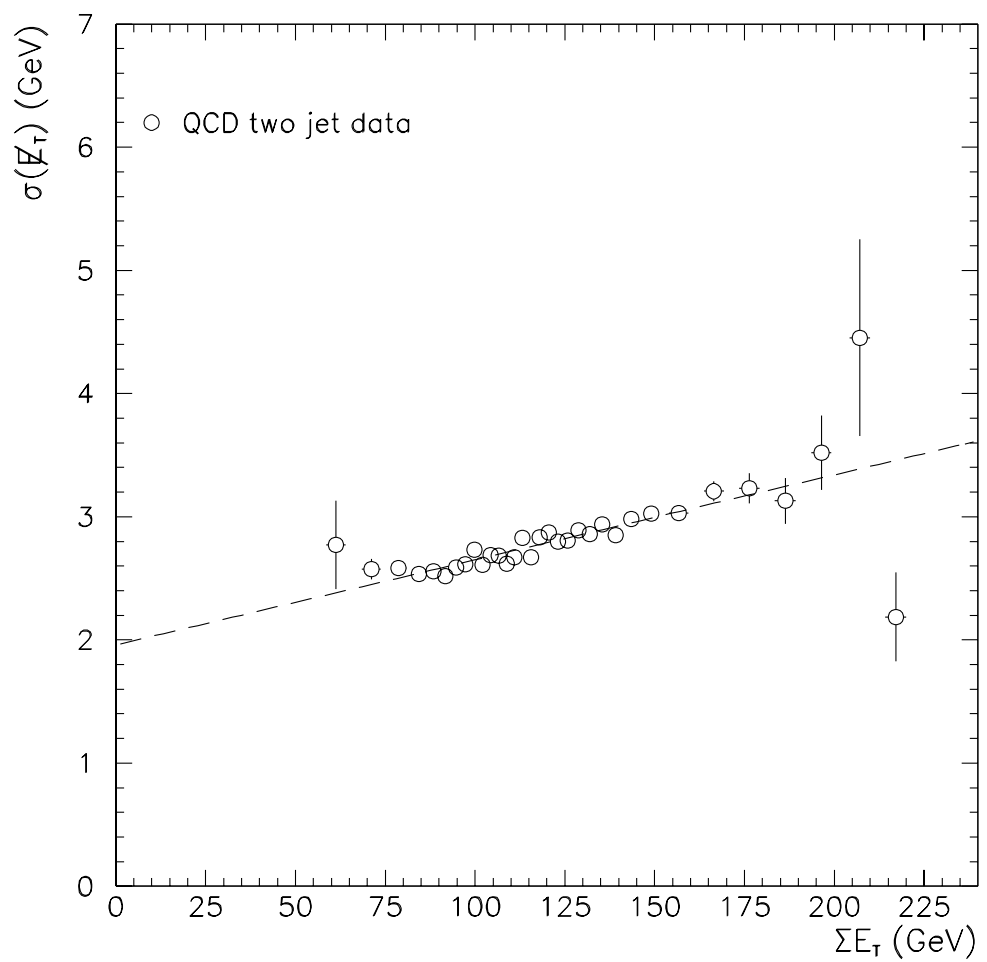

Figure 8.6: $B_{T}$ resolution as a function of total scalar $E_{t}$ as derived from QCD events. 
The transverse mass, $m_{T}$, is then be calculated from these values:

$$
m_{T}=\sqrt{\left(p_{T}^{s}(e)+p_{T}^{s}(\nu)\right)^{2}-p_{T}^{s 2}(W)}
$$

This technique was used to create two different Monte Carlo data sets. Both spanned the $M_{W}$ range from $70-85 \mathrm{GeV}$ in $1 \mathrm{GeV}$ steps with the width kept constant at the Standard Model value of $2.1 \mathrm{GeV}[37]$. The first set, $M C_{T B}$, used an electron response function derived from the testbeam response as shown in Tab. 8.1. The second set applied a response function to the electron that was determined from GEANT (chapter 6). The response function is given in Tab. 8.2. Two more Monte Carlo samples were generated. The first $M C_{\eta}^{\prime}$, contained the GEANT generated response function shifted by 1 standard deviation to investigate the uncertainty in the mass fits. The second, $M C_{0}$, incorporated a response function that was independent of pseudorapidity. This sample was used to investigate the effects of using some intermediate response function.

\subsection{Collider Data Set}

To determine the systematic differences inherent in fitting $M_{W}$ using the different response functions, a data sample from the 1992 - 93 collider run of the $\mathrm{D} \emptyset$ detector was used. The trigger requirements for the data are shown in Tab. 8.3 and defined in more detail in appendix A. Requirements were made on the electron energy at both triggering levels. In addition, level 2 required 


\begin{tabular}{||ccc||}
\hline$\eta$ & Relative Response $(\mathrm{R})$ & $\delta \mathrm{R}$ \\
\hline 0.025 & 0.9767 & 0.0036 \\
0.075 & 0.9775 & 0.0018 \\
0.125 & 0.9883 & 0.0020 \\
0.175 & 0.9896 & 0.0013 \\
0.225 & 0.9968 & 0.0057 \\
0.275 & 0.9954 & 0.0045 \\
0.325 & 1.0001 & 0.0060 \\
0.375 & 0.9986 & 0.0048 \\
0.425 & 0.9994 & 0.0030 \\
0.475 & 1.0006 & 0.0053 \\
0.525 & 0.9983 & 0.0062 \\
0.575 & 0.9982 & 0.0073 \\
0.625 & 1.0014 & 0.0086 \\
0.675 & 1.0013 & 0.0075 \\
0.725 & 1.0063 & 0.0043 \\
0.775 & 1.0098 & 0.0028 \\
0.825 & 1.0139 & 0.0033 \\
0.875 & 1.0162 & 0.0010 \\
0.925 & 1.0180 & 0.0063 \\
0.975 & 1.0193 & 0.0051 \\
\hline
\end{tabular}

Table 8.1: The $\eta$ dependent response function $\mathrm{R}$ used to smear the Monte Carlo data sample $M C_{T B}$.

\begin{tabular}{||ccc||}
\hline$\eta$ & Relative Response $(\mathrm{R})$ & $\delta \mathrm{R}$ \\
\hline 0.05 & 1.0040 & 0.00058 \\
0.15 & 1.0030 & 0.00056 \\
0.25 & 1.0030 & 0.00059 \\
0.35 & 1.0000 & 0.00060 \\
0.45 & 1.0000 & 0.00085 \\
0.55 & 0.9950 & 0.00082 \\
0.65 & 0.9920 & 0.00083 \\
0.75 & 0.9900 & 0.00061 \\
0.85 & 0.9839 & 0.00063 \\
0.95 & 0.9779 & 0.00086 \\
1.05 & 0.9739 & 0.00080 \\
\hline
\end{tabular}

Table 8.2: The $\eta$ dependent response function $\mathrm{R}$ used to smear the Monte Carlo data sample $M C_{\eta}$ and $M C_{\eta}^{\prime}$. 


\begin{tabular}{||cc||}
\hline Trigger & Requirement \\
\hline Level 1 & 1 EM trigger tower $>10 \mathrm{GeV}$ \\
Level 2 & 1 reconstructed electron $>20 \mathrm{GeV}$ \\
& $\left|H_{T}\right|>20 \mathrm{GeV}$ \\
& with isolation cone $=0.4$ \\
\hline Offline & $H_{\text {matrix }} \leq 100$ \\
& Track match $<10$ \\
& $\mathrm{EM}_{\text {frac }} \geq 0.9$ \\
$\mathrm{HC}>0.1$ \\
$\mathrm{MR}<0.4$ \\
\\
$E_{T}^{e} \geq 20 \mathrm{GeV}$ \\
$\mathbb{H}_{T} \geq 20 \mathrm{GeV}$ \\
$40 \leq m_{T} \leq 110 \mathrm{GeV}$ \\
$p_{T}^{W} \leq 20 \mathrm{GeV}$ \\
$N_{\text {cells }}^{E M}>20$ \\
\hline
\end{tabular}

Table 8.3: Triggering requirements for the $\mathrm{W}^{ \pm}$data set[40]. The isolation cone requirement is explained in the text. For a further discussion, refer to appendix A.

an isolation cut:

$$
\frac{E M_{1-5}(\Delta R=0.4)-E M_{1-4}(\Delta R=0.2)}{E M_{1-4}(\Delta R=0.2)}<0.15
$$

where $E M_{i-j}(\Delta R)$ is the electron energy sum for layers $i-j$ for a cone of radius $\Delta R=\sqrt{\Delta \eta^{2}+\Delta \phi^{2}}$. A cut on $\not_{T}$ was also made here $\left(\not_{T}>20 \mathrm{GeV}\right)$.

Offline cuts were also made. These included electron identification cuts. A shower shape cut was applied using data from the testbeam $\left(H_{\text {matrix }}[38]\right)$ and a track match to the central tracker were applied (Track match). In addition, the electron was required to have a certain fraction of its energy deposited in the electromagnetic compartment of the calorimeter $\left(E M_{\text {frac }}\right)$ and to have a minimum number of calorimeter cells included in the energy cluster $\left(N_{\text {cells }}^{E M}\right)$. Requirements to remove anomalous signals due to high voltage discharges and 
interference from the accelerator were made. To remove these signals, energy clusters with a total transverse energy greater than $20 \mathrm{GeV}$ were required to have at least $10 \%$ of their energy in the electromagnetic calorimeter $(\mathrm{HC})$ while all clusters were required to have less than $40 \%$ of their energy in the coarse hadronic compartment (MR). Other cuts were made on the characteristics of the electron, neutrino, and the $\mathrm{W}^{ \pm}$boson. Finally, only events with a transverse mass $\left(m_{T}\right)$ in the range from 40 to $110 \mathrm{GeV}$ were used in the fit.

The distributions of the fitting quantities $m_{T}, p_{T}(e)$, and $p_{T}(\nu)$ were then calculated. A maximum likelihood fit was then performed using each of the Monte Carlo data samples on the data distributions. The results of these fits are given in Tab. 8.4. To investigate the additional uncertainty that the incorrect response function introduces, a third Monte Carlo data sample was generated $\left(M C_{\eta}^{\prime}\right)$ incorporating an $\eta$ response function representing a shift of one standard deviation of the mean response from the function used in $M C_{\eta}$. The same fitting procedure was used as above. The difference between the values of $M_{W}$ obtained using the $M C_{\eta}$ and $M C_{\eta}^{\prime}$ samples is the systematic uncertainty. A summary of the results are given below.

\subsection{Results}

The fits for the transverse mass $\left(m_{T}\right)$, the transverse momentum of the electron $\left(p_{T}(e)\right)$ and the transverse momentum of the neutrino $\left(p_{T}(\nu)\right)$ are shown in Figs. $8.7-8.12$. The values from the fits are shown in Tab. 8.4. All the results show a systematic shift down from the expected $\mathrm{W}^{ \pm}$ 


\section{$M_{w}$ Fit: Testbeam Response}

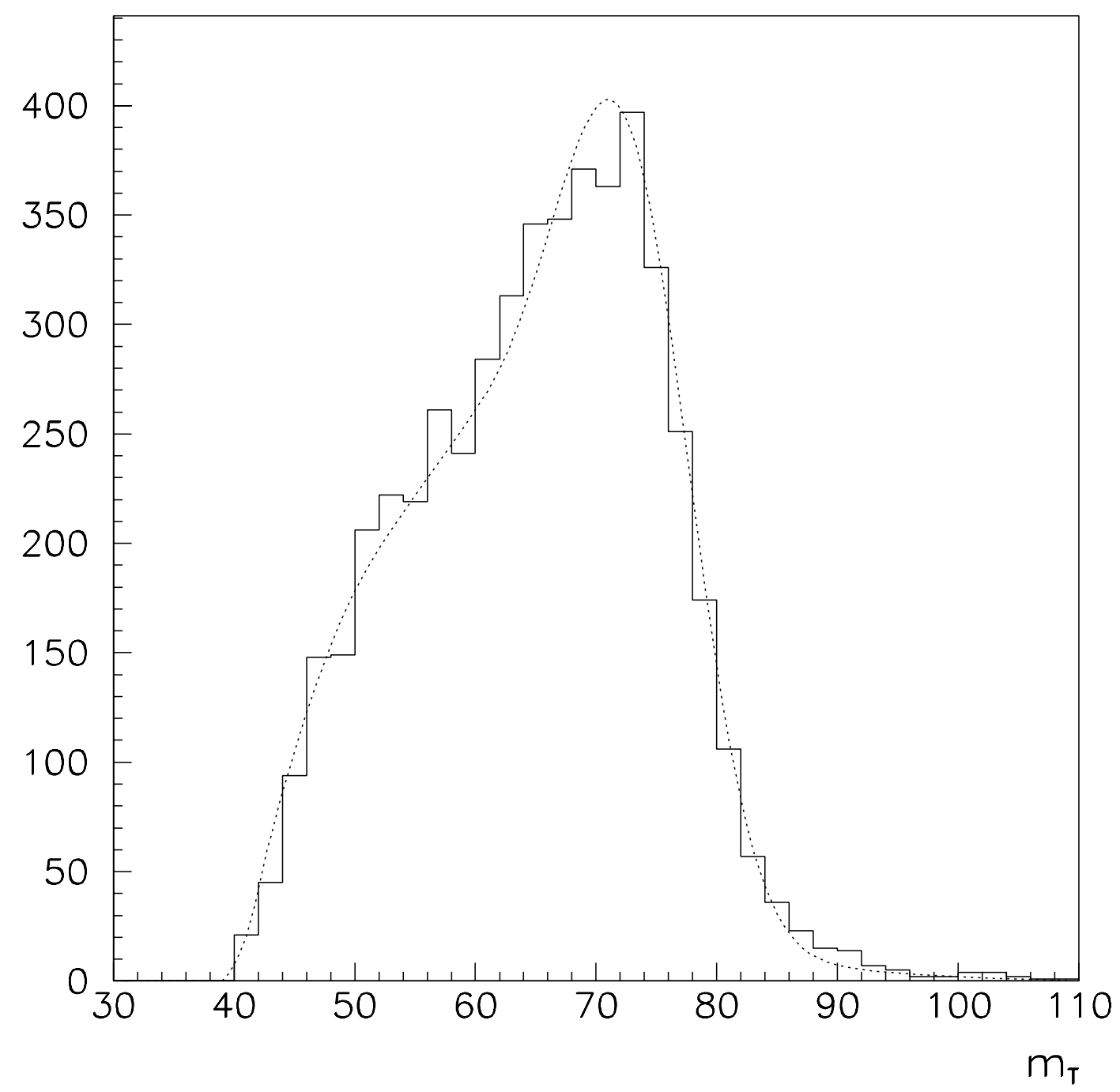

Figure 8.7: Fit for $m_{T}$ with testbeam response function. The histogram contains the data (solid line) while the curve is the Monte Carlo (dotted line). 


\section{$M_{w}$ Fit: Testbeam Response}

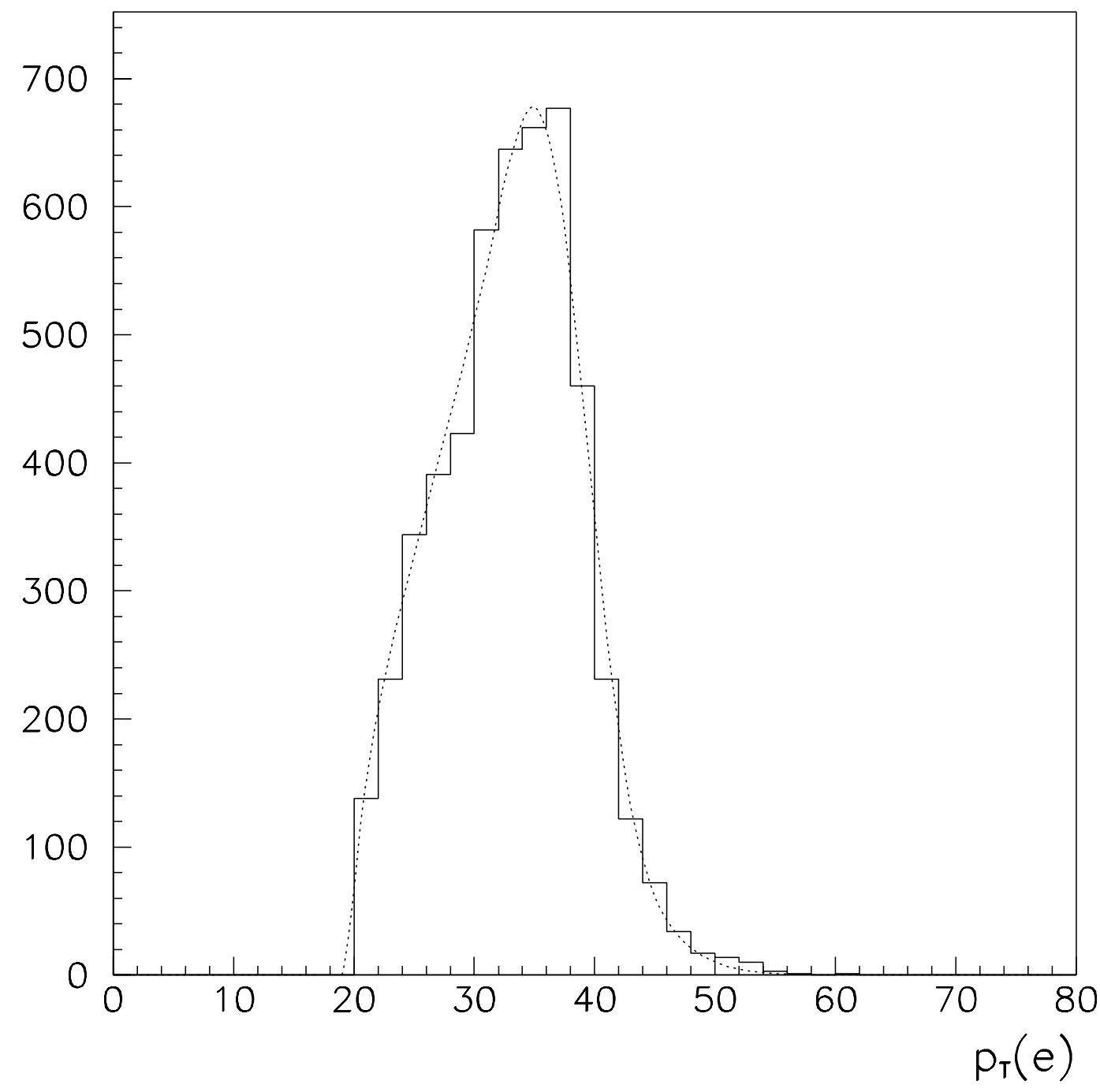

Figure 8.8: Fit for $p_{T}(e)$ with the testbeam response function. The histogram contains the data (solid line) while the curve is the Monte Carlo (dotted line). 


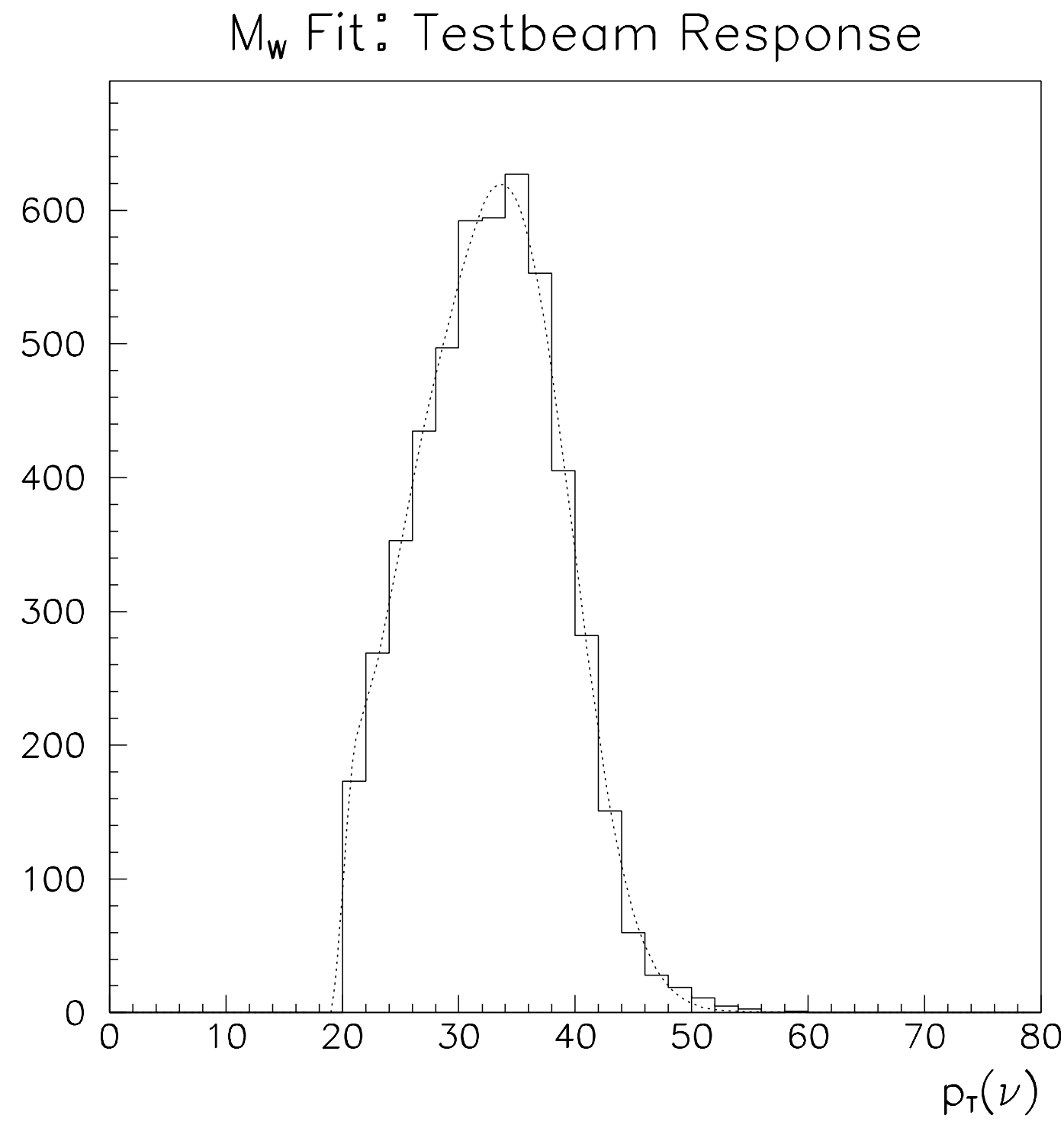

Figure 8.9: Fit for $p_{T}(\nu)$ with the testbeam response function. The histogram contains the data (solid line) while the curve is the Monte Carlo (dotted line). 
$M_{\mathrm{w}}$ Fit: GEANT Response

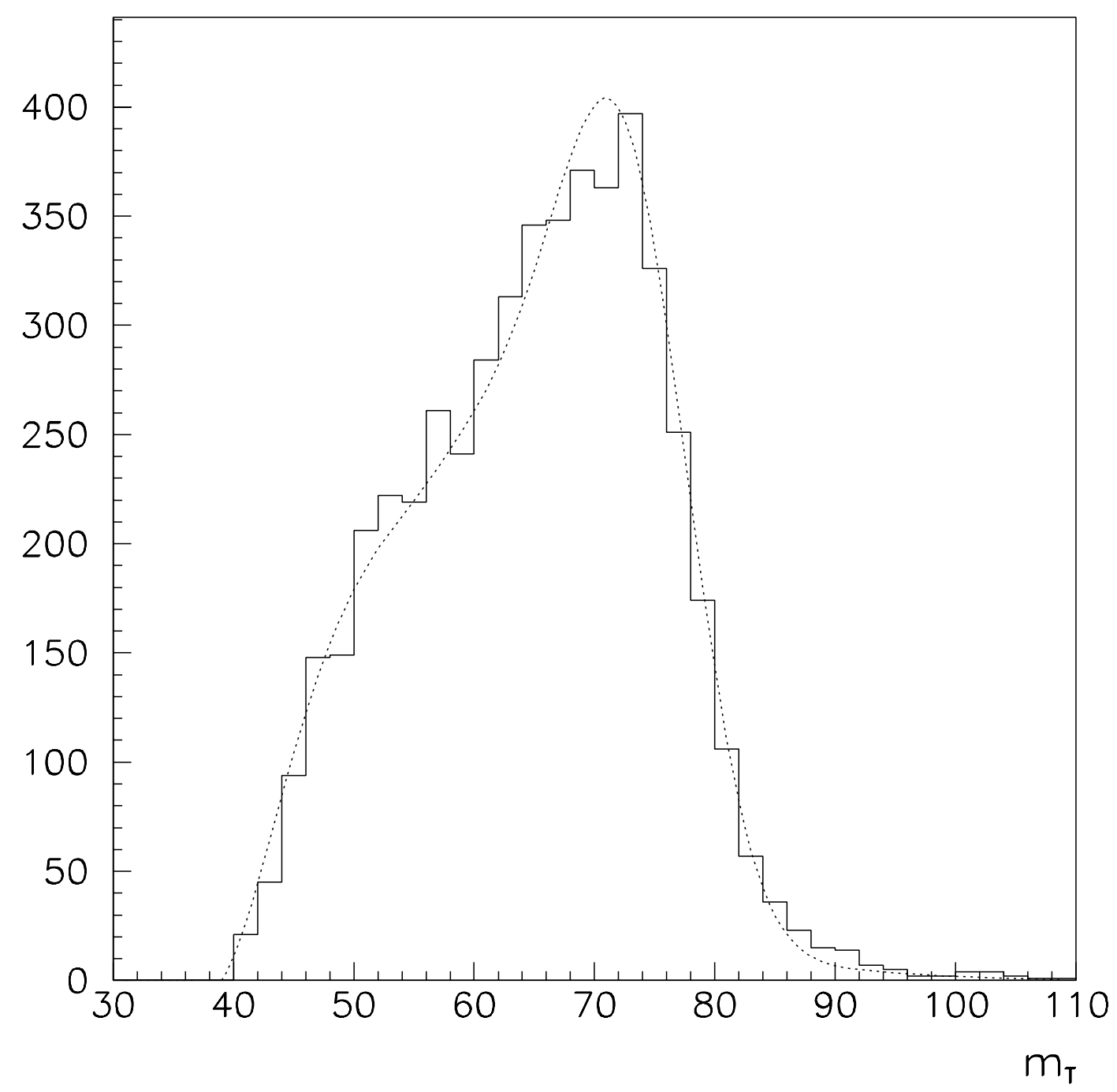

Figure 8.10: Fit for $m_{T}$ with the GEANT $\eta$ response function. The histogram contains the data (solid line) while the curve is the Monte Carlo (dotted line). 
$M_{w}$ Fit: GEANT Response

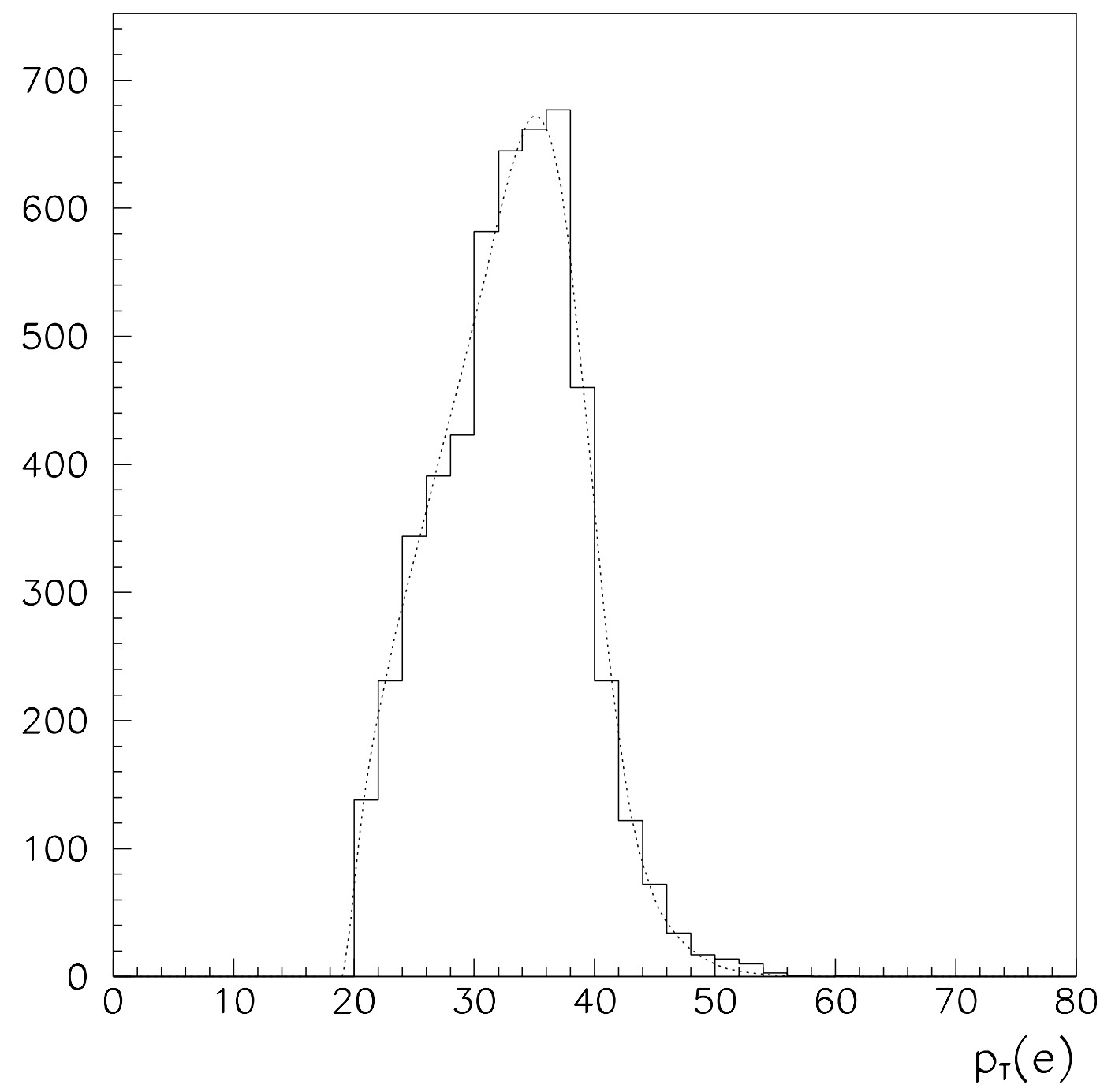

Figure 8.11: Fit for $p_{T}(e)$ with the GEANT $\eta$ response function. The histogram contains the data (solid line) while the curve is the Monte Carlo (dotted line). 


\section{$M_{w}$ Fit: GEANT Response}

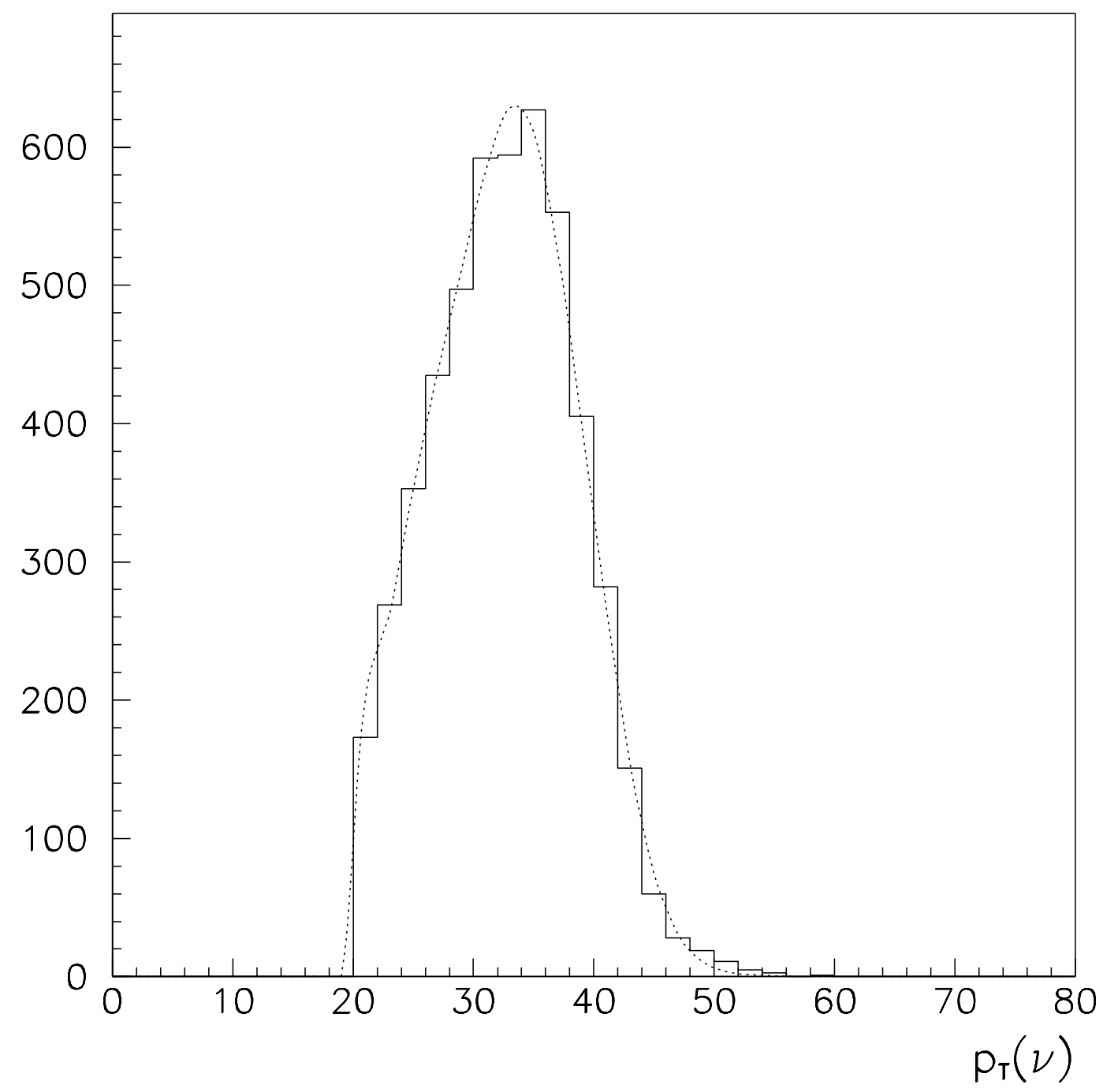

Figure 8.12: Fit for $p_{T}(\nu)$ with the GEANT $\eta$ response function. The histogram contains the data (solid line) while the curve is the Monte Carlo (dotted line). 


\begin{tabular}{|c|c|c|c|}
\hline & $m_{T}$ & $p_{T}(e)$ & $p_{T}(\nu)$ \\
\hline$M C_{T B}$ & & & \\
\hline$M_{W}(\mathrm{GeV})$ & $75.09 \pm 0.14$ & $75.52 \pm 0.21$ & $73.99 \pm 0.23$ \\
\hline $\begin{array}{l}\chi_{d o f}^{2} \\
M C_{\eta}\end{array}$ & 1.65 & 3.17 & 2.09 \\
\hline$M_{W}(\mathrm{GeV})$ & $75.67 \pm 0.17$ & $76.20 \pm 0.20$ & $74.45 \pm 0.31$ \\
\hline $\begin{array}{l}\chi_{d o f}^{2} \\
M C_{0}\end{array}$ & 1.82 & 4.64 & 2.56 \\
\hline$M_{W}(\mathrm{GeV})$ & $75.26 \pm 0.16$ & $75.73 \pm 0.20$ & $74.03 \pm 0.25$ \\
\hline$\chi_{d o f}^{2}$ & 1.77 & 3.69 & 2.19 \\
\hline
\end{tabular}

Table 8.4: Results for the $M_{W}$ fits both with and without the $\eta$ resolution functions. The quoted errors are statistical only.

mass of $\sim 80 \mathrm{GeV}$. This is suspected to be due to a problem with the overall energy scale of the detector caused by an error in transferring the calibration from the testbeam to the full detector. Possible sources of the error include differences in the liquid argon purity between the testbeam cryostat and those at $\mathrm{D} \emptyset$. Temperature differentials between the cryostats could also cause a shift. These and other possible sources of the problem are being investigated by the collaboration. Initial studies of other resonances $(\Upsilon)$ suggest that it is merely a scale problem[39]. This thesis is concerned not with this overall scale factor, but with the systematic bias induced by a response function that has an incorrect dependence on pseudorapidity.

The qualities of the fits are not very good, especially for the electron fit. The poor quality of the fits and the scatter in the fitted mass values suggest that we do not fully understand our detector behavior at this preliminary stage. The best fits are derived for the Monte Carlo data sample generated using the testbeam derived response function. The fits are worse for the flat response 


\begin{tabular}{||cccc||}
\hline & $m_{T}$ & $p_{T}(e)$ & $p_{T}(\nu)$ \\
\hline$\Delta M_{W}(\mathrm{MeV})$ & $580 \pm 60$ & $680 \pm 60$ & $460 \pm 70$ \\
\hline
\end{tabular}

Table 8.5: Systematic shifts in $M_{W}$ introduced by the $\eta$ response function. The errors are taken from the $M C_{\eta}^{\prime}$ data sample.

function and even worse for the response function derived from GEANT.

The systematic shift introduced by the response function is shown in Tab. 8.5. A shift of $460-680 \mathrm{MeV}$ is introduced in the mass determination by the $3 \%$ variation in response. The errors on these shifts are small, $\sim 60 \mathrm{MeV}$. Although the fitted masses determined from $M C_{T B}$ vary by more than their errors, the mass shift introduced by $M C_{\eta}$ is seen in all three of the fits. A similar shift of lesser magnitude is also seen in the $M C_{0}$ fits.

One recommendation to solve the problem of understanding the response of the electromagnetic calorimeter would be to use the EGS4 simulation instead of GEANT. As we showed in chapter 7, EGS does a superior job of reproducing the behavior of the detector. Unfortunately, EGS does not posses the ability to model complicated detector systems. Incorporating it into a Monte Carlo of the full $D \emptyset$ detector would be difficult, but would most likely provide a better simulation. 


\section{Chapter 9}

\section{Conclusion}

In 1991, the $\mathrm{D} \emptyset$ collaboration conducted a testbeam concentrating on detector modules from their central calorimeter. In parallel, an effort was put forth to model the testbeam configuration using GEANT to allow for the transfer of the calibration from the testbeam to the collider. This simulation produced mixed results.

GEANT is a standard in high energy physics detector simulation. We saw that it is able to reproduce the resolution function of the $D \emptyset$ uranium/liquid argon calorimeter for electrons. We also saw that it did not model well the behavior of the response function as a function of pseudorapidity. Using the results of the Monte Carlo in the collider environment to determine $M_{W}$ leads to a large systematic bias on the order of $\sim 450-700 \mathrm{MeV}$.

Another useful Monte Carlo is EGS4. EGS4 was able to model the behavior of the response of the detector to beams of electrons at various pseudorapidities. Using this simulation in place of GEANT to transfer the testbeam calibration by properly modeling the upstream material in the collider environ- 
ment would eliminate the systematic bias caused by using the flawed GEANT response function.

Simulations are important in high energy physics for both understanding detector behavior and interpreting signals to extract results. They provide a method to extrapolate testbeam studies to situations where the use of calibration beams is not practical. Although very useful, these simulations are never perfect. It is important to understand their strengths and weaknesses so that their abilities are not exceeded. 


\section{Bibliography}

[1] DØ Design Report, The DØ Collaboration (1983, revised 1984,1985) unpublished.

[2] W.J. Marciano, "Quantitative Tests of the Standard Model of Electroweak Interactions", BNL-45999.

W.J. Marciano, "Precision Tests of Electroweak Theory", BNL-47278.

[3] G. Arnison, et al., Phys. Lett. 122B (1983) 103.

[4] G. Arnison, et al., Phys. Lett. 126B (1983) 398.

[5] M. Banner, et al., Phys. Lett. 122B (1983) 476.

[6] P. Bagnaia, et al., Phys. Lett. 129B (1983) 130.

[7] S.L. Glashow, Nucl. Phys. 22 (1961) 579.

S. Weinberg, Phys. Rev. Lett. 19 (1967) 1264.

A. Salam, "Elementary Particle Theory", ed. N. Svartholm (Stockholm: Almqvist, Forlag A B) p. 367.

[8] F. Halzen and A.D. Martin, Quarks and Leptons: An Introductory Course in Modern Particle Physics, John Wiley \& Sons, Inc., 1984. 
[9] D. Decamp, et al., Phys. Lett. 246B (1990) 306.

[10] F. Abe, et al., Phys. Rev. D45 (1992) 3921.

[11] M.Z. Akrawy, et al., "Measurement of the $\mathrm{Z}^{0}$ Mass and Width with the OPAL Detector at LEP", Phys. Lett. 231B (1989) 530.

D. Decamp, et al., Phys. Lett. 235B (1990) 399.

[12] V. Barger, J.L. Hewett, and T.G. Rizzo, Phys. Rev. D42 (1990) 152.

[13] J. Alitti, et al., Phys. Lett. 241B (1990) 150.

[14] F. Abe, et al., Phys. Rev. D43 (1991) 2070.

[15] Amaldi, U., "Fluctuations in Calorimetry Measurements",in T. Ferbel (ed.), Experimental Techniques in High Energy Physics, Addison-Wesley Publishing Company, Inc., 1987.

[16] Particle Data Group, Phys. Rev. D45 (1992) III.14.

[17] C. Fabjan, "Calorimetry in High Energy Physics", in T. Ferbel (ed.), Experimental Techniques in High Energy Physics, Addison-Wesley Publishing Company, Inc., 1987.

[18] R. Wigmans, Nucl. Instrum. Methods A259 (1987) 389.

R. Wigmans, "Advances in Hadron Calorimetry", Annu. Rev. Nucl. Part. Sci. 41 (1991) 133.

[19] E. Bernardi, "On the Optimization of the Energy Resolution of Hadron 
Calorimeters",, Ph.D. Thesis, zur Erlangung des Doktorgrades des Fachbereichs Physik der Universität Hamburg, Hamburg, Germany, (1987).

[20] S. Abachi, et al., "The DØ Detector", to be published in NIM.

[21] M. Tartaglia, "Measurement of the DØ Test Beamline Momentum using Cerenkov Pressure Curves", DØ Note 1660.

[22] R. Brun, et al., "GEANT3", CERN, DD/EE/84-1.

[23] H. Fenker, Fermilab Internal Note, TM-1179, 1983.

[24] K. Hodel, private communication.

[25] S. Feher and D. Stewart, private communication.

[26] K. Streets, “The DØ Test Beam Load 2 Gain Calculation", DØ Note 1600.

[27] W.G.D. Dharmaratna, T. Heuring, “CCEM Response vs $\eta$ - Test Beam Load 2", DØ Note 1577.

[28] W.R. Nelson, H. Hirayama and D.W.O. Rogers, “The EGS4 Code System", SLAC Report 265.

[29] T. Heuring, "GEANT 3.14 vs. EGS4: A Comparison Using the DØ Uranium/Liquid Argon Calorimeter Geometry", to be published in Procedings of the MC93 Conference, World Scientific Publishing Company.

[30] F. Carminati, private communication. 
[31] P.B. Arnold and R.P. Kauffman, "W and Z Production at Next-to-leading Order: from Large $q_{T}$ to Small", Nucl. Phys. B349 (1991) 381.

[32] G. Blaylock and D. Wood, "Analysis of $p_{T}$ Spectra of W and Z Bosons", UA2 Note 598, February, 1990.

[33] K. Einsweiler, "The Measurement of the $\mathrm{W}$ and $\mathrm{Z}$ Masses at UA2", UA2 Note 600, March, 1990.

[34] L. Fayard, D. Froidevaux, and D. Wood, "New Analysis of the W and Z Masses", UA2 Note 620, June, 1991.

[35] S. Rajagopalan, private communication.

[36] M.F. Paterno, private communication.

[37] Particle Data Group, Phys. Rev. D45 (1992) II.1.

[38] N. Graf, private communication.

[39] M. Rijssenbeek, private communication.

[40] M. Demarteau, DØ Note 1677.

[41] Y. Xia, private communication.

[42] Y. Xia and R. Astur, DØ Note 869, July, 1989.

[43] A. Boehnlein, DØ Note 1664, March, 1993.

[44] R. Engelmann, et al., NIM 216 (1983) 45-56. 
[45] D. Kewley, et al., NIM A290 (1990) 346-352.

[46] N. Graf, private communication. 


\section{Appendix A}

\section{Trigger and Analysis Cuts}

\section{A.1 Level 1 Trigger Requirements}

The level one trigger requirements used in the accumulation of the $\mathrm{W}^{ \pm}$ data sample in this study were very simple. They consisted of requiring only one calorimeter electromagnetic trigger tower to have at least $10 \mathrm{GeV}$ of transverse energy. The trigger towers consist of four ganged readout towers, the readout towers being 0.1 by 0.1 in $\eta$ and $\phi$, making a trigger tower 0.2 by 0.2 . The trigger towers are subdivided into the electromagnetic compartment and the hadronic compartment. Analog signals are taken from the BLS's (section 3.2.4) and added using precision resistors scaling the signal by the nominal $\sin \theta$. This trigger requirement focuses on finding an electron candidate. 


\section{A.2 Level 2 Trigger Requirements}

The level 2 had access to the entire event structure in order to make its decision. For $\mathrm{W}^{ \pm}$events, the requirements were that the level 2 tools, software written to find specific particles or event characteristics, find an electron with a transverse energy of at least $20 \mathrm{GeV}$, that it pass an isolation criterion, and that the reconstructed transverse energy imbalance be at least $20 \mathrm{GeV}$. This trigger again focuses on the electron, looking for a clean, high $p_{T}$ candidate, but also looks for signs that the event contained a neutrino.

The electron tool uses information from the calorimeter and the output of the level 1 trigger. Level 1 passes a list of electromagnetic trigger towers that exceed threshold, in this case $10 \mathrm{GeV}$. Level 2 then passes through this list, unpacking the stored energy information for the cells in a 3 by 3 trigger tower window ( 0.6 in both $\eta$ and $\phi)$ about the passed tower. The cell with the highest energy in the third layer of the electromagnetic section is taken as the seed for subsequent tests. It then uses this information to determine whether the energy cluster has a high probability of being an electron.

Level 2 first makes a determination if the electron candidate is of high enough energy to continue testing. It sums all the electromagnetic cells in a 3 by 3 readout tower window ( 0.3 in both $\eta$ and $\phi)$ about the seed cell, and using the vertex determined from level 0 , calculates the transverse energy of the electron. If this quantity exceeds the threshold, the testing continues. Otherwise, it proceeds to the next candidate. Additional tests made on successful candidates include comparing the longitudinal and transverse shower shapes 
to testbeam and Monte Carlo data. The total candidate energy is defined to be the sum of the energies in the electromagnetic layers and the first fine hadronic layer in a 0.3 by $0.3 \eta, \phi$ window centered on the seed. An electron is required to have a certain fraction $(\sim 90 \%)$ of its energy deposited in the electromagnetic section. The quantity

$$
E M 3_{\text {frac }}=\frac{E M 3}{E M T O T}
$$

where $E M 3$ is the energy deposited in the third electromagnetic layer and EMTOT is the total candidate energy in the electromagnetic section, is also required to be in some range dependent on the candidates energy and pseudorapidity. Finally, to look at the transverse shower shape, the difference between the sum of the energy in the third electromagnetic layer for a .25 by . $25 \eta$ by $\phi$ window and a .15 by .15 window is required to match that of testbeam electrons[41][42]

An isolation cut is also made on the electron candidate. In this case, the energy in two concentric cones centered on the original seed are compared. The ratio:

$$
I S O(0.4)=\frac{E M T O T(\Delta R=0.4)-E M_{1-4}(\Delta R=0.2)}{E M_{1-4}(\Delta R=0.2)}
$$

where $E M T O T$ is the total energy of the electron candidate including the first layer of the fine hadronic section in some cone $\Delta R=\sqrt{\Delta \eta^{2}+\Delta \phi^{2}}$ and $E M_{1-4}$ is the energy sum for only the electromagnetic section, is required to satisfy some criterion as derived from testbeam data $(I S O(0.4)<0.15)[41]$.

To identify the neutrino, level 2 looks at the $\mathbb{E}_{T}$. The algorithm looks at 
the $x$ and $y$ components separately:

$$
\begin{gathered}
E_{x}=\sum_{i} E_{i} Z_{c o r r} \sin \theta \cos \phi \\
E_{y}=\sum_{i} E_{i} Z_{c o r r} \sin \theta \sin \phi \\
H_{T}=\sqrt{E_{x}^{2}+E_{y}^{2}}
\end{gathered}
$$

where $Z_{\text {corr }}$ is the vertex correction factor. Anomalous signals due to noisy cells are removed from the sum if the ratio:

$$
H C=\frac{\left(E_{T}^{u p}+E_{T}^{\text {down }}\right)}{N} / E_{T}^{i}
$$

where $E_{T}^{\text {up/down }}$ is the transverse energy of the cell directly above or below the candidate cell $\left(E_{T}^{i}\right)$ in the readout tower and $N$ is the number of neighboring cells, is less then $0.05[43][36]$

\section{A.3 Analysis Requirements}

The data set that was passed through the two levels of triggering were then written to tape for further analysis. In this analysis, further requirements were made on the data. These included shower shape cuts, track matching, corrections for anomalous signals, and kinematic cuts. The cuts will be discussed in this section.

The offline shower shape cuts are more sophisticated than those in level 2. Both the longitudinal and the transverse shower shapes are compared simultaneously to shapes from testbeam data. This comparison takes advantage 
of the correlations in the shower profiles by constructing a covariance matrix $M_{i j}[44][45]:$

$$
M_{i j}=\frac{1}{N} \sum_{n=1}^{N}\left(x_{i}^{(n)}-\bar{x}_{i}\right)\left(x_{j}^{(n)}-\overline{x_{j}}\right)
$$

where the sum is over a set of $N$ events and $x_{i}$ a quantity related to the electron shower such as the energy in cell $i$. This matrix is then inverted,

$$
H \equiv M^{-1}
$$

and a variable $\zeta_{m}$ defined:

$$
\zeta_{m} \equiv \sum_{i j}\left(x_{i}^{m}-\bar{x}_{i}\right) H_{i j}\left(x_{j}^{m}-\bar{x}_{j}\right)
$$

for an event $m$ to characterize how much the event is like an electron.

The matrix $H$, frequently called the $\mathrm{H}$ matrix, has a dimensionality of 41 in this case. The elements include the fractional energies in EM layers 1, 2, and 4, the energies for EM layer 3 in a 6 cell by 6 cell window about the EM layer 3 cell with the most energy, the logarithm of the total energy, and the primary $\mathrm{z}$ vertex position. We require the electron to have a $\zeta \leq 100$, which is $95 \%$ efficient for electrons[46]. To remove any anomalous signals, a requirement is made on the number of calorimeter cells that make up the electron candidate. We require $N_{\text {cells }}^{E M}>20$. Real electrons have a very sharp cutoff at 20 in their distributions of number of cells[46]. An additional longitudinal shower shape requirement, identical to one made at level 2 , is made to require that at least $90 \%$ of the energy of the electron be deposited in the electromagnetic section of the calorimeter. 
Requirements were made on matching the track from the central drift chamber to the shower centroid in the calorimeter. A quantity called track significance, $s$ is defined:

$$
s^{2} \equiv\left(\frac{\Delta r \phi}{\delta_{r \phi}}\right)^{2}+\left(\frac{\Delta z}{\delta_{z}}\right)^{2}
$$

where $\Delta r \phi$ and $\Delta z$ are the difference between the track from the central drift chamber and the calorimeter in the $r \phi$ and $z$ planes respectively, and $\delta_{i}$ is the error on these measurements as derived from testbeam and collider data[46]. The significance was required to have a value $s<10$.

Anomalous signals were removed using two different cuts. For energy clusters with a $E_{T}>20 \mathrm{GeV}$, at least $10 \%$ of the energy was required to be in the electromagnetic section of the calorimeter. For all jets, the fraction of energy in the coarse hadronic section of the calorimeter was required to be less than $40 \%$. Both of these cuts reduce the number of spurious signals in the calorimeter by requiring realistic longitudinal shower shapes[35]. A correction was made to account for the fact that the testbeam calibration and the full detector are maintained at different high voltages. This correction amounts to $\sim 1.5 \%[46]$.

Kinematic cuts were applied to the event. These included the electron $\left(E_{T}^{e} \geq 20 \mathrm{GeV}\right)$, the neutrino $\left(\not_{T} \geq 20 \mathrm{GeV}\right)$, the $\mathrm{W}^{ \pm}\left(p_{T}^{W} \leq 20 \mathrm{GeV}\right)$, and the transverse mass $\left(40 \leq m_{T} \leq 110 \mathrm{GeV}\right)$. The same kinematic requirements were made in the Monte Carlo data set. 